\title{
Investigation into the Organobismuth Dismutation and Its Use for Rational Synthesis of Heteroleptic Triarylbismuthines, $\operatorname{Ar}^{1}{ }_{2} \mathrm{Ar}^{2} \mathrm{Bi}$
}

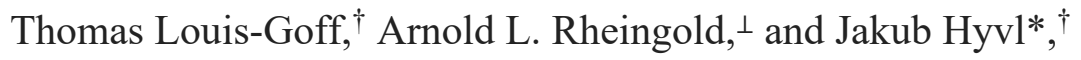 \\ 'Department of Chemistry, University of Hawai'i at Mānoa, 2545 McCarthy Mall, Honolulu, Hawaii 96822, United \\ States \\ ${ }^{\perp}$ Department of Chemistry, University of California, San Diego, 9500 Gilman Drive, La Jolla, California 92093, \\ United States \\ Contact: hyvl@hawaii.edu
}

1. General Comments on Experimental Details - S2

2. Experimental Details for the Synthesis of Organobismuths, $\mathbf{2}$ and $\mathbf{3}$ - S3

3. Experimental Details for the Preparation of Various Organometallic Reagents and their use in the Synthesis of Diphenyl(4-(dimethoxymethyl)phenyl)bismuthane (1a) in Table 1-S4

4. Experimental Details for the Investigation of Dismutation, Table S1 - S5

5. Experimental Details for the Synthesis of Heteroleptic Triarylbismuthanes 1 -S6

6. EA Reports and NMR Spectra for 3b, Figures S1-S5 - S13

7. EA Reports, NMR Spectra, and HPLC Chromatograms for Compounds 1, Figures S6-S78 - S18

8. HPLC Chromatogram of tri(4-methoxyphenyl)bismuthane, di(4-methoxyphenyl) phenylbismuthane $\mathbf{1} \mathbf{j}$, diphenyl(4-methoxyphenyl)bismuthane $\mathbf{1 b}$, and triphenylbismuthane, Figure S79 - S91

9. Crystal Structure Data for (4-cyanophenyl)(p-tolyl)bismuthane 1k, Figure S80, Tables S2-S6 S92

10. References $-\mathrm{S} 100$ 


\section{General Comments on Experimental Section}

All reactions were carried out under nitrogen atmosphere. All reagents and solvents were purchased from commercial suppliers. $\mathrm{BiCl}_{3}$ and $\mathrm{ZnCl}_{2}$ were purified by reflux with thionyl chloride, then dissolved in diethyl ether and filtered to remove impurities. $p$-Toluene-sulfonic acid monohydrate $\left(p-\mathrm{TsOH} \cdot \mathrm{H}_{2} \mathrm{O}\right)$ was purified by co-distillation in toluene. All other reagents were used as purchased without further purification. Solvents were purified through an alumina column solvent system and further dried with molecular sieves. Column chromatography was performed with 35-70 mesh silica gel using flash column techniques or Combiflash $\odot$.NextGen System. Varian Unity Inova $500 \mathrm{MHz}$ was used for recording the ${ }^{1} \mathrm{H}$ and ${ }^{13} \mathrm{C}$ NMR spectra. Chemical shifts for ${ }^{1} \mathrm{H}$ and ${ }^{13} \mathrm{C}$ and were given in part per million (ppm), referenced internally according to the residual solvent resonances. Coupling constants were given in $\mathrm{Hertz}(\mathrm{Hz})$ and the following abbreviations were used: s, singlet; $d$, doublet; $t$, triplet; $m$, multiplet.

Diphenylbismuth chloride $\left(\mathrm{Ph}_{2} \mathrm{BiCl}\right)^{1}$, diphenylbismuth iodide $\left(\mathrm{Ph}_{2} \mathrm{BiI}\right)^{2}$ and dimesitylbismuth iodide $\mathbf{2 b}^{2}$ were prepared according to previously reported procedures.

${ }^{13} \mathrm{C}$ DEPT spectra are color coded and reported as follows: Blue: DEPT 135; Green: DEPT 90; Red: DEPT 45.

\section{Instrumentation:}

${ }^{1} \mathrm{HNMR}$ and ${ }^{13} \mathrm{CNMR}$ were collected on a Varian Unity Inova $500 \mathrm{MHz}$. EA samples were analyzed with a PerkinElmer 2400 Series II Analyzer at the University of Rochester by Bill Brennessel. HPLC chromatograms were collected on an Agilent 1200 series using a 25 minute 25:75 to 0:100 $\mathrm{H}_{2} \mathrm{O}$ :Acetonitrile gradient on a Discovery ${ }^{\circledR} \mathrm{C} 1825 \mathrm{~cm}$ x $4 \mathrm{~mm}, 5 \mu \mathrm{m}$. 


\section{Experimental Details for the Synthesis of Organobismuths, 2 and 3}

\section{Diphenylbismuth tosylate (2a)}

The synthetic protocol was modified from a previously reported procedure. ${ }^{3}$ To a diethyl ether solution of triphenylbismuthane $(20.0 \mathrm{~g}, 45.4 \mathrm{mmol})$ was added dropwise a solution of $p$ - $\mathrm{TsOH} \cdot \mathrm{H}_{2} \mathrm{O}(8.64 \mathrm{~g}, 45.4$ $\mathrm{mmol}$ ) in diethyl ether. The reaction mixture was allowed to stir for 5-6 hours, then filtered and the collected solid washed two times with diethyl ether to afford $20.8 \mathrm{~g}, 86 \% \mathbf{2 a}$.

\section{Phenylbismuth ditosylate (3a)}

The protocol was modified from a previously reported procedure. ${ }^{3}$ To a diethyl ether solution of triphenylbismuthane $(1.0 \mathrm{~g}, 2.3 \mathrm{mmol}$ in $10 \mathrm{ml})$ was added dropwise a solution of $p-\mathrm{TsOH} \cdot \mathrm{H}_{2} \mathrm{O}(0.91 \mathrm{~g}, 4.7$ mmol). The reaction mixture was stirred at $90{ }^{\circ} \mathrm{C}$ for 3 hours, then cooled to room temperature, filtered, and the collected solid washed two times with diethyl ether $(40 \mathrm{ml})$ affording quantitatively phenylbismuth ditosylate (3a).

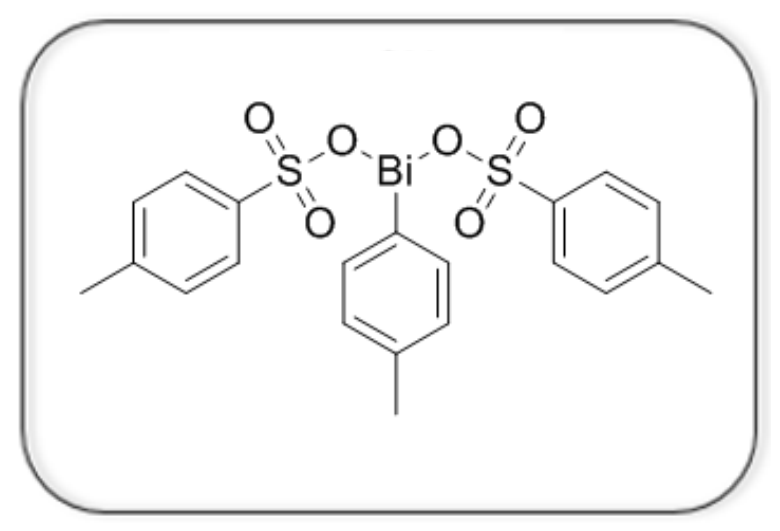

p-Tolylbismuth ditosylate (3b): A solution of $p$ $\mathrm{TsOH} \cdot \mathrm{H}_{2} \mathrm{O}(2.22 \mathrm{~g}, 11.7 \mathrm{mmol})$ was added slowly to a solution of tri( $p$-tolyl)bismuthane $(2.77 \mathrm{~g}, 5.75 \mathrm{mmol})$ in diethyl ether. The reaction mixture stirred at $90{ }^{\circ} \mathrm{C}$ for 5 hours, then cooled to room temperature, filtered, and the collected solid washed two times with diethyl ether $(25 \mathrm{ml})$ affording the product $(3.65 \mathrm{~g}, 5.69 \mathrm{mmol})$ in $99 \%$ yield.

White Powder. ${ }^{1} \mathbf{H}$ NMR (dd-DMSO): $\delta 8.57$ (d, $\left.J=7.6 \mathrm{~Hz}, 2 \mathrm{H}, \mathrm{C}_{6} H_{4}-\mathrm{CH}_{3}, \mathrm{CH}\right), 7.83(\mathrm{~d}, J=7.6 \mathrm{~Hz}, 2 \mathrm{H}$, $\mathrm{C}_{6} H_{4}-\mathrm{CH}_{3}, \mathrm{CH}$ ), 7.48 (d, J=7.7 Hz, 4H, $\left.A r \mathrm{SO}_{3} \mathrm{Bi}, \mathrm{CH}\right), 7.11$ (d, J=7.7 Hz, 4H, $\left.A r S \mathrm{SO}_{3} \mathrm{Bi}, \mathrm{CH}\right), 2.28$ (overlapping singlets, 9H, $\left.\mathrm{C}_{6} \mathrm{H}_{4}-\mathrm{CH}_{3}\right) .{ }^{13} \mathbf{C}$ NMR (d 6 -DMSO): $\delta$ 145.8, 138.2, 137.2, $136.8(\mathrm{Ar}-\mathrm{Bi}, \mathrm{CH})$, 134.0 ( $A r-\mathrm{Bi}, \mathrm{CH}), 132.7,128.5\left(\mathrm{ArSO} \mathrm{SO}_{3} \mathrm{Bi}, \mathrm{CH}\right), 125.9\left(\mathrm{ArSO} \mathrm{SO}_{3} \mathrm{Bi}, \mathrm{CH}\right), 21.9\left(\mathrm{C}_{6} \mathrm{H}_{4}-\mathrm{CH}_{3}\right), 21.2\left(\mathrm{C}_{6} \mathrm{H}_{4}-\mathrm{CH}_{3}\right)$. Anal. Calc. for $\mathbf{B i O}_{6} \mathbf{C}_{21} \mathbf{H}_{21} \mathbf{S}_{2}$ : C, 39.26; H, 3.29. Found: C, 38.87; H, 2.87.

3b did not pass EA in \%H. EA was consistently low in $\% \mathrm{C}$ and $\% \mathrm{H}$. 


\section{Experimental Details for the Preparation of Various Organometallic Reagents and their use in the Synthesis of Diphenyl(4-(dimethoxymethyl)phenyl)bismuthane (1a) in Table 1.}

\section{ArLi}

4-bromobenzaldehyde dimethylacetal (260 $\mathrm{mg}, 1.12 \mathrm{mmol}$ ) was added to a Schlenk flask and dissolved in $5 \mathrm{ml} \mathrm{THF}$, then cooled to $-78{ }^{\circ} \mathrm{C}$. A solution of $n$-butyllithium in hexanes $(1.12 \mathrm{mmol}, 0.7 \mathrm{ml})$ was added dropwise to the reaction solution and stirred at $-78^{\circ} \mathrm{C}$ for 40 minutes. Then, diphenylbismuth tosylate $(0.936$ mmol) was added and the reaction mixture was allowed to warm to $-10^{\circ} \mathrm{C}$. The final concentration was kept to $0.075 \mathrm{M}$ of the nucleophile in THF. The reaction was stirred for 1 hour and 40 minutes. Afterwards, the reaction mixture was allowed to warm to ambient temperature.

\section{Work-up}

Then, the reaction mixture was quenched with a saturated solution of $\mathrm{NaHCO}_{3}$ in distilled water and diluted with EtOAc $(15 \mathrm{ml})$, upon which the two phases were separated. The aqueous phase was washed twice with EtOAc $(10 \mathrm{ml})$. The combined organic phases were washed twice with sat. $\mathrm{NaHCO}_{3}(10 \mathrm{ml})$ and twice with sat. brine solution (10ml). Finally, the organic phase was dried over $\mathrm{MgSO}_{4}$, filtered through silica gel, and concentrated in vacuo. The crude reaction mixture was then purified by column chromatography using a 20:1 hexanes : ethyl acetate eluent.

\section{$\left[\mathrm{Ar}_{2} \mathrm{Cu}\right] \mathrm{Li}$}

To a freshly prepared solution of 4-lithium benzaldehyde dimethyl acetal solution $(2.25 \mathrm{mmol}, 0.075 \mathrm{M})$ at $-78{ }^{\circ} \mathrm{C}$ was added $\mathrm{CuI}(1.12 \mathrm{mmol})$ and the solution was warmed to $-40^{\circ} \mathrm{C}$ and stirred for 1 hour. To this organocuprate reagent was added diphenyl bismuth tosylate $(0.936 \mathrm{mmol})$ and warmed to $-10{ }^{\circ} \mathrm{C}$. The final concentration was kept to $0.075 \mathrm{M}$ of the nucleophile in THF. The reaction was stirred for 1 hour and 40 minutes. Afterwards, the reaction was worked up as previously described.

\section{$\operatorname{ArMgBr}$}

A modified preparation of Grignard reagent was utilized to prepare a stock solution of organomagnesium reagent. Magnesium turnings $(7.9 \mathrm{~g}, 325 \mathrm{mmol})$ were added to a Schlenk bomb and to it was added $100 \mathrm{ml}$ THF. 4-Bromobenzaldehyde dimethylacetal $(130 \mathrm{mmol})$ was dissolved in THF $(50 \mathrm{ml})$ and added dropwise to the magnesium. The mixture was allowed to stir for $30 \mathrm{mins}$, then heated to $65{ }^{\circ} \mathrm{C}$ for 4 hours.

A solution of organomagnesium reagent $(1.12 \mathrm{mmol})$ was cooled to $-10{ }^{\circ} \mathrm{C}$. To this solution was added diphenylbismuth tosylate $(0.936 \mathrm{mmol})$ to give a final concentration of $0.075 \mathrm{M}$ of the nucleophile in THF. The reaction was stirred for 1 hour and 40 minutes. Afterwards, the reaction was worked up as previously described.

\section{$\left[\mathrm{Ar}_{2} \mathrm{Cu}\right] \mathrm{MgX}$}

A solution of Grignard reagent $(2.25 \mathrm{mmol})$ was cooled to $-40{ }^{\circ} \mathrm{C}$ and to it was added $\mathrm{CuI}(1.12 \mathrm{mmol})$ and stirred for one hour. To this organocuprate reagent was added diphenylbismuth tosylate $(0.936 \mathrm{mmol})$ to give a final concentration of $0.075 \mathrm{M}$ of the nucleophile in THF. The reaction was warmed to $-10{ }^{\circ} \mathrm{C}$ and stirred for 1 hour and 40 minutes. Afterwards, the reaction was worked up the same as previously described. 


\section{$[\mathrm{ArCuCN}] \mathrm{MgX}$}

A solution of organomagnesium reagent $(1.12 \mathrm{mmol})$ was cooled to $-40{ }^{\circ} \mathrm{C}$ and to it was added $\mathrm{CuCN}(1.12$ $\mathrm{mmol}$ ) and stirred for one hour. To this organocuprate reagent was added diphenylbismuth tosylate (0.936 mmol) to give a final concentration of $0.075 \mathrm{M}$ of the nucleophile in THF. The reaction was warmed to $10{ }^{\circ} \mathrm{C}$ and stirred for 1 hour and 40 minutes. Afterwards, the reaction was worked up the same as previously described.

\section{$\operatorname{ArZnX}$}

To a solution of Grignard reagent (1.12 mmol) was added anhydrous $\mathrm{ZnCl}_{2}(153 \mathrm{mg}, 1.12 \mathrm{mmol})$ dissolved in $5 \mathrm{ml}$ THF. The reaction was stirred at room temperature for about 10 minutes, then cooled to $-10{ }^{\circ} \mathrm{C}$. Next, diphenylbismuth tosylate $(0.936 \mathrm{mmol})$ was added to the reaction mixture to give a final concentration of $0.075 \mathrm{M}$ of the nucleophile in THF. The reaction was allowed to stir at $-10{ }^{\circ} \mathrm{C}$ for 1 hour and 40 minutes. Afterwards, the reaction was worked up the same as previously described.

\section{Experimental Details for the Investigation of Dismutation}

\section{Investigation of Dismutation Initiated by 10mol\% Additive (Scheme 2)}

To a stirring THF (2mL) solution of diphenyl(4-methoxylphenyl)bismuthane 1b, (100mg, $0.213 \mathrm{mmol}$ ), a THF $(1 \mathrm{~mL})$ solution of $10 \mathrm{~mol} \%$ additive $(0.0213 \mathrm{mmol})$ was added and stirred for one hour. After one hour, the mixture was filtered through Celite ${ }^{\circ}$ and THF was removed in vacuo and the products were separated by Combiflash. The isolated yields are as follows ( $\mathrm{Ph}_{2} \mathrm{BiAnisyl}$ (1b), $\mathrm{Ph}_{3} \mathrm{Bi}$ (A), $\mathrm{Anisyl}_{2} \mathrm{BiPh}(\mathrm{B})$, $\left.\operatorname{Anisyl}_{3} \mathrm{Bi}(\mathrm{C})\right)$ :

Table S1: Recovery and Molar Ratio of Dismutation Side Products Initiated by 10 mol\% Various Additives to diphenyl(4-methoxylphenyl)bismuthane 1b

\begin{tabular}{|c|c|c|c|c|c|c|c|}
\hline Entry & Additive & $\begin{array}{c}1 \mathrm{~b} \\
(\mathrm{mg})\end{array}$ & $\begin{array}{c}\mathrm{A} \\
(\mathrm{mg})\end{array}$ & $\begin{array}{c}\mathrm{B} \\
(\mathrm{mg})\end{array}$ & $\begin{array}{c}\mathrm{C} \\
(\mathrm{mg})\end{array}$ & Recovery 1b\% & $\begin{array}{c}\text { 1b:A:B:C } \\
\text { (molar ratios) }\end{array}$ \\
\hline 1 & $\mathrm{PhMgBr}$ & 99 & - & - & - & 99 & - \\
\hline 2 & $\mathrm{PhZnBr}$ & 98 & - & - & - & 98 & - \\
\hline 3 & $\mathrm{MgCl}_{2}$ & 95 & - & - & - & 95 & - \\
\hline 4 & $\mathrm{ZnCl}_{2}$ & 89 & - & - & - & 89 & $11.3: 9.2: 5.7: 1$ \\
\hline 5 & $\mathrm{Ph}_{2} \mathrm{BiCl}$ & 30 & 23 & 16 & 3 & 30 & $17.5: 15.1: 5.6: 1$ \\
\hline 6 & $\mathrm{Ph}_{2} \mathrm{BiI}$ & 43 & 35 & 14 & 2.7 & 43 & $18.6: 15.1: 3.7: 1$ \\
\hline 7 & $\mathrm{Ph}_{2} \mathrm{BiOTs}$ & 33 & 25 & 7 & 2 & 33 & \\
\hline
\end{tabular}

\section{Investigation of Dismutation Initiated by the Slow Addition of Electrophile into Nucleophile}

To a stirring THF (2mL) solution of nucleophile, $p$-MeOC6H4ZnX (0.408 mmol), a THF (0.25 mL) solution of diphenylbismuth iodide (50mg; $0.102 \mathrm{mmol}$ ), was added four times in half hour intervals to give a final volume of $3 \mathrm{ml}$ and an equimolar ratio of electrophile to nucleophile. After the final addition and stirring for 30 minutes, the reaction was then quenched with sat. $\mathrm{NaHCO}_{3}$ aqueous solution and extracted with ethyl acetate $2 \times 10 \mathrm{ml}$. The combined organic phase was washed with sat. $\mathrm{NaHCO}_{3}(2 \times 10 \mathrm{ml})$, dried 
over $\mathrm{MgSO}_{4}$, and the solvent was removed in vacuo. Finally, the crude product was purified through a silica gel plug and the products were separated by Combiflash. The isolated yields were as follows: $\mathrm{Ph}_{2} \mathrm{BiAnisyl}$ (1b): 105mg (0.223 mmol, 55\%); $\mathrm{Ph}_{3} \mathrm{Bi}$ (A): 6mg (0.0136 mmol, 3\%); Anisyl $_{2} \mathrm{BiPh}$ (B): 8mg (0.0160 mmol, 4\%).

\section{Investigation of Dismutation Initiated by the Slow Addition of Nucleophile into Electrophile}

To a stirring THF $(2 \mathrm{~mL})$ solution of diphenylbismuth iodide $(200 \mathrm{mg} ; 0.408 \mathrm{mmol})$, a THF $(0.25 \mathrm{~mL})$ solution of nucleophile, $p$-MeOC6H4ZnX $(0.102 \mathrm{mmol})$, was added four times in half hour intervals to give a final volume of $3 \mathrm{ml}$ and an equimolar ratio of electrophile to nucleophile. After the final addition and stirring for 30 minutes, the reaction was then quenched with sat. $\mathrm{NaHCO}_{3}$ aqueous solution and extracted with ethyl acetate $2 \mathrm{x} 10 \mathrm{ml}$. The combined organic phase was washed with sat. $\mathrm{NaHCO}_{3}(2 \mathrm{x} 10 \mathrm{ml})$, dried over $\mathrm{MgSO}_{4}$, and the solvent was removed in vacuo. Finally, the crude product was purified through a silica gel plug and the products were separated by Combiflash. The isolated yields were as follows: $\mathrm{Ph}_{2} \mathrm{BiAnisyl}$ (1b): 60mg (0.128 mmol, 31\%); $\mathrm{Ph}_{3} \mathrm{Bi}(\mathrm{A}): 42 \mathrm{mg}$ (0.0954 mmol, 23\%); Anisyl $_{2} \mathrm{BiPh}$ (B): 23mg (0.0460 mmol, 11\%).

\section{Experimental Details for the Synthesis of Heteroleptic Triarylbismuthanes (1)}

\section{General Procedure Utilizing Organozincs and Diarylbismuth Tosylate or Iodide (2) (Procedures A and $B$ )}

For compounds 1a, 1c, 1e, 1f, $\mathbf{1 h}$, organozinc reagent was prepared by the addition of anhydrous $\mathrm{ZnCl}_{2}$ $(153 \mathrm{mg}, 1.12 \mathrm{mmol})$ dissolved in $5 \mathrm{ml}$ THF to a solution of Grignard reagent $(1.12 \mathrm{mmol})$. For compounds 1b, 1g, an organozinc reagent was prepared by Knochel's TurboGrignard procedure. ${ }^{4}$ Compound 1d was prepared utilizing Rieke procedures. ${ }^{5}$

The volume of solution of organozinc reagent $(1.12 \mathrm{mmol})$ was adjusted to a final volume of $15 \mathrm{ml}$ by addition of dry THF. The reagent was stirred at room temperature for about 10 minutes, then cooled to -10 ${ }^{\circ} \mathrm{C}$. After that, diarylbismuth tosylate (Procedure A) or iodide (Procedure B) $2(0.936 \mathrm{mmol})$ was added at once to a stirred organozinc solution. The reaction mixture was stirred for 1 hour and 40 minutes and allowed to warm to room temperature. Then, the reaction was quenched by addition of a saturated solution of $\mathrm{NaHCO}_{3}$ in distilled water and extracted with EtOAc $(15 \mathrm{ml})$. The aqueous phase was washed twice with EtOAc $(10 \mathrm{ml})$. The combined organic phases were washed twice with saturated $\mathrm{NaHCO}_{3}(10 \mathrm{ml})$ and twice with saturated brine solution $(10 \mathrm{ml})$. Finally, the organic phase was dried over $\mathrm{MgSO}_{4}$, filtered through silica gel and concentrated in vacuo affording crude product. Further purification or recrystallization methods are described below for each compound.

\section{General Procedure Utilizing Organozincs and Arylbismuth Ditosylates (3) (Procedure C)}

Regarding compounds $\mathbf{1 i}$ and $\mathbf{1 k}$, an organozinc reagent was prepared by Knochel protocol. ${ }^{4}$ For compounds $\mathbf{1} \mathbf{j}$ and $\mathbf{1}$, organozinc reagent was prepared by the addition of anhydrous $\mathrm{ZnCl}_{2}(293 \mathrm{mg}, 2.15$ $\mathrm{mmol}$ ) dissolved in $5 \mathrm{ml}$ THF to a solution of Grignard reagent $(2.06 \mathrm{mmol})$. 
The volume of the organozinc reagent $(2.06 \mathrm{mmol})$ was adjusted by addition of THF to give a final volume of $15 \mathrm{ml}$, then subsequently cooled to $-10{ }^{\circ} \mathrm{C}$ and stirred. The monoarylbismuth ditosylate $(0.936 \mathrm{mmol})$ was added at once to the stirred organozinc solution at $-10{ }^{\circ} \mathrm{C}$ and the reaction mixture was stirred for 1 hour and 40 minutes and then allowed to warm to room temperature. The work up is identical to Procedure A. Further purification or recrystallization methods are described below for each compound.

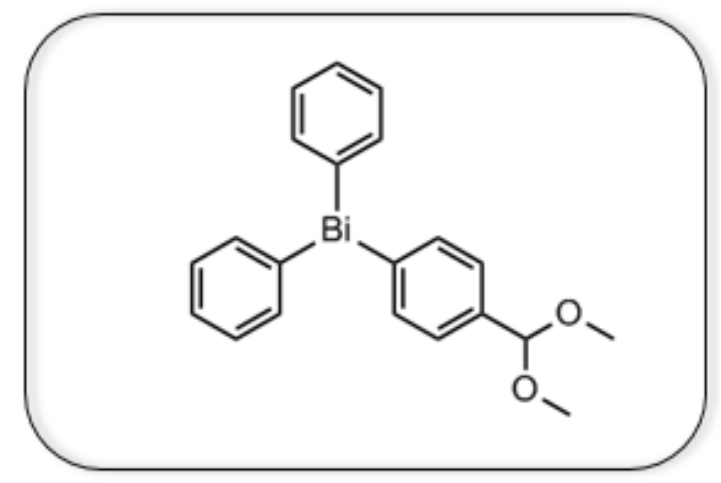

Diphenyl(4-dimethoxymethylphenyl)bismuthane (1a): Yield: $94 \%$. This compound was obtained according to procedure A from (4-benzaldehyde dimethyl acetal)magnesium(II) bromide $(1.123 \mathrm{mmol})$ and diphenyl bismuth tosylate $(0.5 \mathrm{~g}, 0.936 \mathrm{mmol})$. Purified by chromatography and eluted with a gradient mixture of solvents (hexane/ethyl acetate $100 / 0$ to 20/80).

White solid. ${ }^{1} \mathbf{H}$ NMR ( $\left.\mathbf{C D C l}_{3}\right): \delta 7.78-7.75\left(\mathrm{~m}, 6 \mathrm{H}, \mathrm{C}_{6} H_{5}\right), 7.48\left(\mathrm{~d}, J=7.9 \mathrm{~Hz}, 2 \mathrm{H}, \mathrm{C}_{6} H_{4}-\mathrm{CH}\left(\mathrm{OCH}_{3}\right)_{2}\right)$, 7.40 (m, 4H, Ar), 7.35-7.32 (m, 2H, $\left.\mathrm{C}_{6} H_{5}\right), 5.37$ (s, $1 \mathrm{H}, \mathrm{C}_{6} \mathrm{H}_{4}-\mathrm{CH}\left(\mathrm{OCH}_{3}\right)_{2}, 3.35\left(\mathrm{~s}, 6 \mathrm{H}, \mathrm{C}_{6} \mathrm{H}_{4}-\mathrm{CH}\left(\mathrm{OCH}_{3}\right)_{2}\right)$. ${ }^{13} \mathbf{C ~ N M R}\left(\mathbf{C D C l}_{3}\right): \delta 155.4,155.0,137.5,137.5(\mathrm{Ar}, \mathrm{CH}), 137.4\left(C_{6} \mathrm{H}_{5}, C \mathrm{H}\right), 130.5(\mathrm{Ar}, \mathrm{CH}), 128.7\left(C_{6} \mathrm{H}_{4}^{-}\right.$ $\left.\mathrm{CH}\left(\mathrm{OCH}_{3}\right)_{2}, \mathrm{CH}\right), 127.7\left(\mathrm{C}_{6} \mathrm{H}_{5}, \mathrm{CH}\right), 103.3\left(\mathrm{C}_{6} \mathrm{H}_{4}-\mathrm{CH}\left(\mathrm{OCH}_{3}\right)_{2}, 52.8\left(\mathrm{C}_{6} \mathrm{H}_{4}-\mathrm{CH}\left(\mathrm{OCH}_{3}\right)_{2}\right)\right.$. Anal. Calc. for $\mathbf{B i O}_{2} \mathbf{C}_{21} \mathbf{H}_{21}$ : C, 49.04; H, 4.12. Found: C, 48.76; H, 3.96.

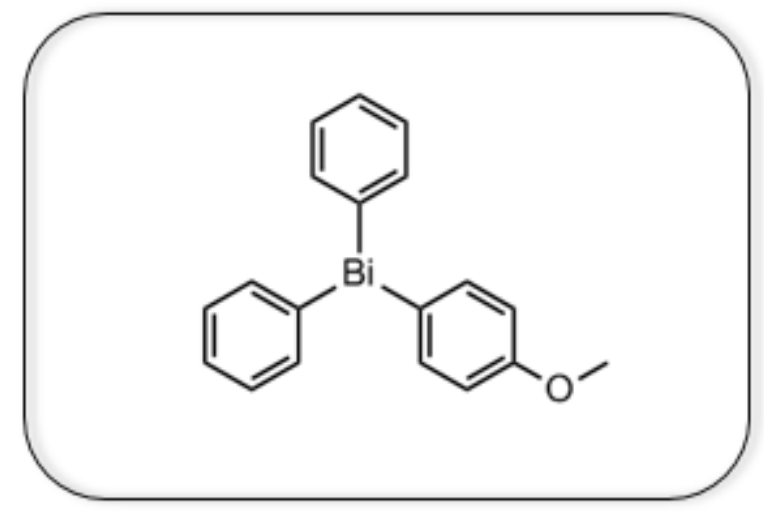

\section{Diphenyl(4-methoxylphenyl)bismuthane}

(1b): Yield: $99 \%$. This compound was obtained according to procedure A from (4-methoxyphenyl)magnesium(II) bromide $(1.123 \mathrm{mmol})$ and diphenylbismuth tosylate $(0.5 \mathrm{~g}, 0.936 \mathrm{mmol})$. Purified by chromatography and eluted with a gradient mixture of solvents (hexane/ethyl acetate $100 / 0$ to $95 / 5$.

Yellowish oil. ${ }^{1} \mathbf{H}$ NMR $\left(\mathbf{C D C l}_{3}\right): \delta 7.77\left(\mathrm{~d}, J=7.0 \mathrm{~Hz}, 4 \mathrm{H}, \mathrm{C}_{6} H_{5}\right), 7.68\left(\mathrm{~d}, J=8.5 \mathrm{~Hz}, 2 \mathrm{H}, \mathrm{C}_{6} H_{4}-\mathrm{OCH}_{3}\right)$, $7.42\left(\mathrm{~d}, J=7.0 \mathrm{~Hz}, 4 \mathrm{H}, \mathrm{C}_{6} H_{5}\right), 7.36-7.33\left(\mathrm{~m}, 2 \mathrm{H}, \mathrm{C}_{6} H_{5}\right), 6.96\left(\mathrm{~d}, J=8.5 \mathrm{~Hz}, 2 \mathrm{H}, \mathrm{C}_{6} H_{4}-\mathrm{OCH}_{3}\right), 3.81(\mathrm{~s}, 3 \mathrm{H}$, $\left.\left.\mathrm{C}_{6} \mathrm{H}_{4}-\mathrm{OCH}\right)_{3}\right) .{ }^{13} \mathbf{C}$ NMR $\left(\mathbf{C D C l}_{3}\right): \delta 159.3,154.8,145.7,138.8\left(\mathrm{C}_{6} \mathrm{H}_{4}-\mathrm{OCH}_{3}, \mathrm{CH}\right), 137.4(\mathrm{Ph}, \mathrm{CH}), 130.4$ $(\mathrm{Ph}, \mathrm{CH}), 127.6(\mathrm{Ph}, \mathrm{CH}), 116.4\left(\mathrm{C}_{6} \mathrm{H}_{4}-\mathrm{OCH}_{3}, \mathrm{CH}\right), 55.0\left(\mathrm{C}_{6} \mathrm{H}_{4}-\mathrm{OCH}_{3}\right)$. Anal. Calc. for $\mathbf{B i O C}_{19} \mathrm{H}_{17}$ : $\mathrm{C}$, 48.52; H, 3.64. Found: C, 48.65; H, 3.68. 


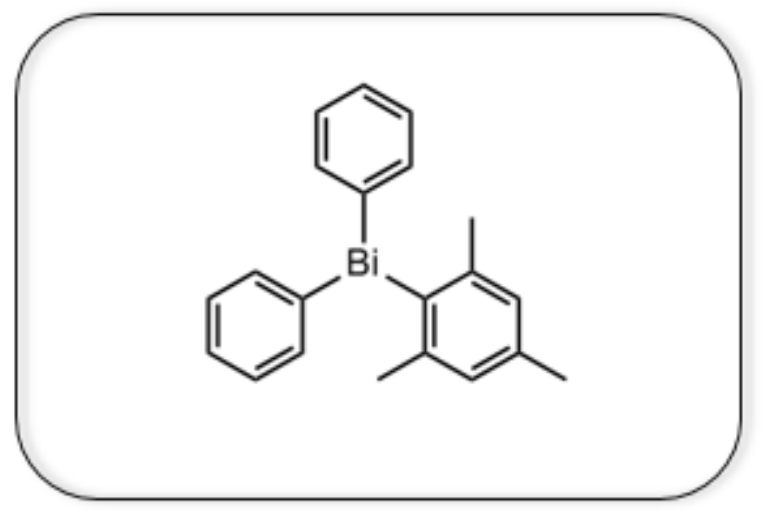

Diphenyl(2-mesityl)bismuthane (1c): Yield: 97\%. This compound was obtained according to procedure A from (mesityl)magnesium(II) bromide (1.123 mmol) and diphenyl bismuth tosylate $(0.5 \mathrm{~g}, 0.936$ $\mathrm{mmol})$. Recrystallized from cold hexanes.

White solid. ${ }^{1} \mathbf{H}$ NMR (CDCl $)$ : $\delta 7.86\left(\mathrm{~d}, J=6.5 \mathrm{~Hz}, 4 \mathrm{H}, \mathrm{C}_{6} H_{5}\right), 7.40-7.37\left(\mathrm{~m}, 4 \mathrm{H}, \mathrm{C}_{6} H_{5}\right), 7.32-7.29(\mathrm{~m}$, $\left.2 \mathrm{H}, \mathrm{C}_{6} H_{5}\right), 7.04\left(\mathrm{~s}, 2 \mathrm{H}, \mathrm{C}_{6} \mathrm{H}_{2}\right), 2.31\left(\mathrm{~s}, 3 \mathrm{H}, \mathrm{C}_{6} \mathrm{H}_{2}-p \mathrm{CH}_{3}\right), 2.26\left(\mathrm{~s}, 6 \mathrm{H}, \mathrm{C}_{6} \mathrm{H}_{2}-o \mathrm{CH}_{3}\right) .{ }^{13} \mathbf{C ~ N M R}\left(\mathbf{C D C l}_{3}\right): \delta$ 157.4, 152.1, 146.2, 138.2, $137.5(\mathrm{Ph}, \mathrm{CH}), 130.2(\mathrm{Ph}, \mathrm{CH}), 129.3\left(\mathrm{C}_{6} \mathrm{H}_{2}, C \mathrm{H}\right), 127.4(\mathrm{Ph}, \mathrm{CH}), 28.5\left(\mathrm{C}_{6} \mathrm{H}_{2}-\right.$ $\left.o C \mathrm{H}_{3}\right), 21.1\left(\mathrm{C}_{6} \mathrm{H}_{2}-p C \mathrm{H}_{3}\right)$. Anal. Calc. for $\mathbf{B i C}_{\mathbf{2 1}} \mathbf{H}_{21}$ : C, 52.29; H, 4.39. Found: C, 52.19; H, 4.29.

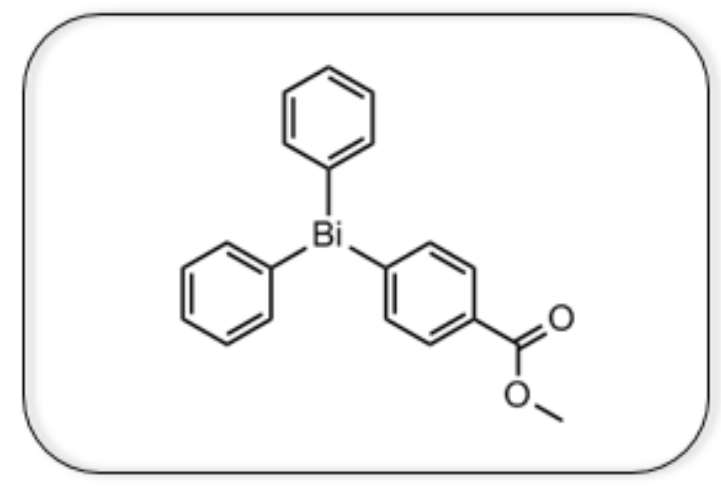

Diphenyl(4-methoxycarbonylphenyl)bismuthane (1d): Yield: $83 \%$. This compound was obtained according to procedure A from (4-methoxycarbonylphenyl)zinc(II) halide $(1.123 \mathrm{mmol})$ and diphenylbismuth tosylate $(0.5 \mathrm{~g}$, $0.936 \mathrm{mmol})$. Recrystallized from cold hexanes.

White solid. ${ }^{1} \mathbf{H}$ NMR $\left(\mathbf{C D C l}_{3}\right): \delta 8.02\left(\mathrm{~d}, J=8.1 \mathrm{~Hz}, 2 \mathrm{H}, \mathrm{C}_{6} H_{4}-\mathrm{COOMe}\right), 7.84\left(\mathrm{~d}, J=8.1 \mathrm{~Hz}, 2 \mathrm{H}, \mathrm{C}_{6} H_{4^{-}}\right.$ COOMe), $7.74\left(\mathrm{~d}, J=6.6, \mathrm{~Hz}, 4 \mathrm{H}, \mathrm{C}_{6} H_{5}\right), 7.43-7.40\left(\mathrm{~m}, 4 \mathrm{H}, \mathrm{C}_{6} H_{5}\right), 7.36-7.32\left(\mathrm{~m}, 2 \mathrm{H}, \mathrm{C}_{6} H_{5}\right), 3.91(\mathrm{~s}, 3 \mathrm{H}$, $\left.\mathrm{C}_{6} \mathrm{H}_{4}-\mathrm{COOCH}_{3}\right) .{ }^{13} \mathbf{C} \mathbf{N M R}\left(\mathbf{C D C l}_{3}\right): \delta 167.3,161.6,155.3,137.6\left(\mathrm{C}_{6} \mathrm{H}_{4}-\mathrm{COOMe}, \mathrm{CH}\right), 137.5(\mathrm{Ph}, \mathrm{CH})$, $131.0\left(\mathrm{C}_{6} \mathrm{H}_{4}-\mathrm{COOMe}, \mathrm{CH}\right), 130.6(\mathrm{Ph}, \mathrm{CH}), 129.4,127.9(\mathrm{Ph}, \mathrm{CH}), 52.1\left(\mathrm{C}_{6} \mathrm{H}_{4}-\mathrm{COOCH}_{3}\right)$. Anal. Calc. for $\mathbf{B i O}_{2} \mathbf{C}_{20} \mathbf{H}_{17}:$ C, 48.20; H, 3.44. Found: C, 48.60; H, 3.26. 


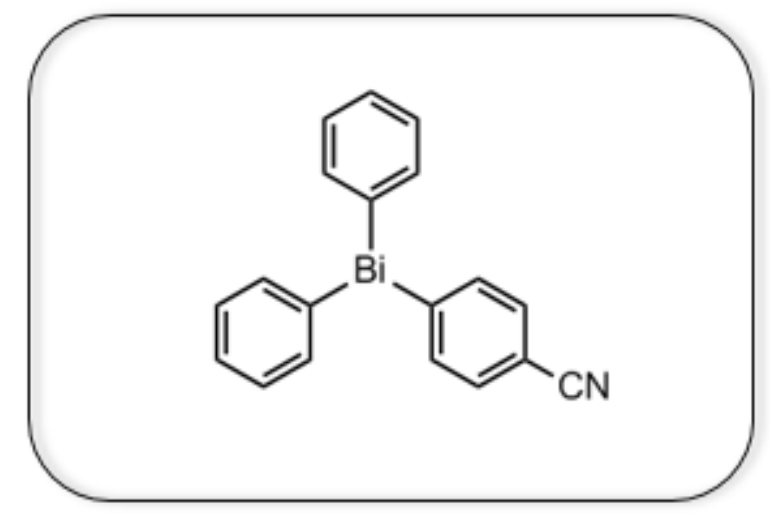

Diphenyl(4-cyanophenyl)bismuthane (1e): Yield: $84 \%$. This compound was obtained according to procedure A from (4-cyanophenyl)zinc(II) halide $(1.123 \mathrm{mmol})$ and diphenylbismuth tosylate $(0.5 \mathrm{~g}$, $0.936 \mathrm{mmol})$. Purified by column chromatography and eluted with a gradient mixture of solvents (hexane/ethyl acetate $100 / 0$ to $25 / 75$.

White solid. ${ }^{1} \mathbf{H}$ NMR (CDCl $)$ ): $\delta 7.87\left(\mathrm{~d}, J=8.1 \mathrm{~Hz}, 2 \mathrm{H}, \mathrm{C}_{6} H_{4} \mathrm{CN}\right), 7.73\left(\mathrm{~d}, J=6.6 \mathrm{~Hz}, 4 \mathrm{H}, \mathrm{C}_{6} H_{5}\right), 7.60$ $\left(\mathrm{d}, J=8.1 \mathrm{~Hz}, 2 \mathrm{H}, \mathrm{C}_{6} H_{4} \mathrm{CN}\right), 7.44\left(\mathrm{t}, J=7.3 \mathrm{~Hz}, 4 \mathrm{H}, \mathrm{C}_{6} H_{5}\right), 7.37\left(\mathrm{~m}, 2 \mathrm{H}, \mathrm{C}_{6} H_{5}\right) .{ }^{13} \mathbf{C} \mathbf{N M R}\left(\mathbf{C D C l}_{3}\right): \delta$ 161.5, 155.7, $138.1\left(\mathrm{C}_{6} \mathrm{H}_{4} \mathrm{CN}, \mathrm{CH}\right), 137.4(\mathrm{Ph}, \mathrm{CH}), 133.2\left(\mathrm{C}_{6} \mathrm{H}_{4} \mathrm{CN}, \mathrm{CH}\right), 130.8(\mathrm{Ph}, \mathrm{CH}), 128.1(\mathrm{Ph}, \mathrm{CH})$, 119.1, 111.3. Anal. Calc. for $\mathbf{B i N C}_{\mathbf{1 9}} \mathbf{H}_{\mathbf{1 4}}$ : C, 49.04; H, 3.03; N, 3.01. Found: C, 49.14; H, 2.96; N, 2.93.

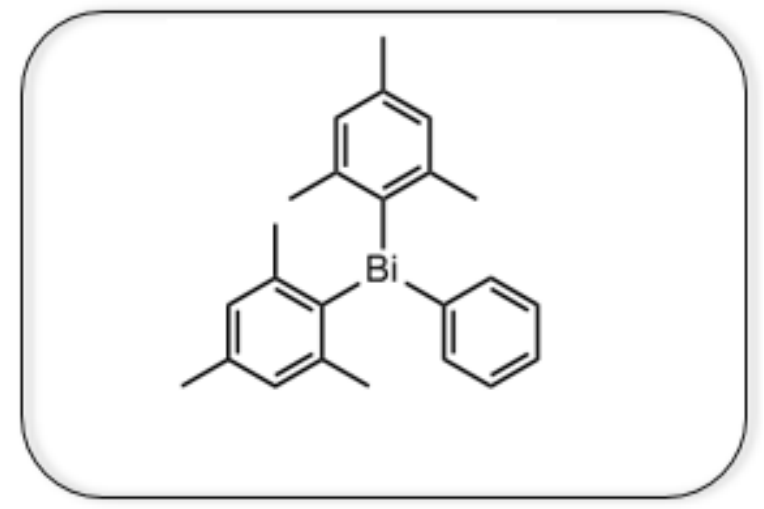

Dimesitylphenylbismuthane (1f): Yield: 94\%. This compound was obtained according to procedure $\mathbf{B}$ from phenylmagnesium(II) bromide $(1.123 \mathrm{mmol})$ and dimesitylbismuth iodide $(0.537 \mathrm{~g}, 0.936 \mathrm{mmol} \mathrm{mmol})$. Recrystallized from hexanes.

White solid. ${ }^{1} \mathbf{H}$ NMR (CDCl $\left.\mathbf{C}_{3}\right): \delta 7.96\left(\mathrm{~d}, J=6.4 \mathrm{~Hz}, 2 \mathrm{H}, \mathrm{C}_{6} H_{5}\right), 7.35-7.32\left(\mathrm{~m}, 2 \mathrm{H}, \mathrm{C}_{6} H_{5}\right), 7.28(\mathrm{~d}, J=7.2$ $\left.\mathrm{Hz}, 1 \mathrm{H}, \mathrm{C}_{6} H_{5}\right), 7.00\left(\mathrm{~s}, 4 \mathrm{H}, \mathrm{C}_{6} H_{2}\right), 2.29$ (overlapping singlets, $\left.18 \mathrm{H}, \mathrm{C}_{6} \mathrm{H}_{2}-\mathrm{CH}_{3}\right) .{ }^{13} \mathbf{C} \mathbf{N M R}\left(\mathbf{C D C l}_{3}\right): \delta$ 155.9, 150.6, 145.5, $138.7(\mathrm{Ph}, \mathrm{CH}), 137.4,129.8(\mathrm{Ph}, \mathrm{CH}), 129.2\left(\mathrm{C}_{6} \mathrm{H}_{2}, \mathrm{CH}\right), 127.1(\mathrm{Ph}, \mathrm{CH}), 27.9\left(\mathrm{C}_{6} \mathrm{H}_{2}-\right.$ $\left.\mathrm{CH}_{3}\right), 21.0\left(\mathrm{C}_{6} \mathrm{H}_{2}-\mathrm{CH}_{3}\right)$. Anal. Calc. for $\mathbf{B i C}_{24} \mathbf{H}_{27}$ C, 54.96; H, 5.19. Found: C, 54.94; H, 4.80. 


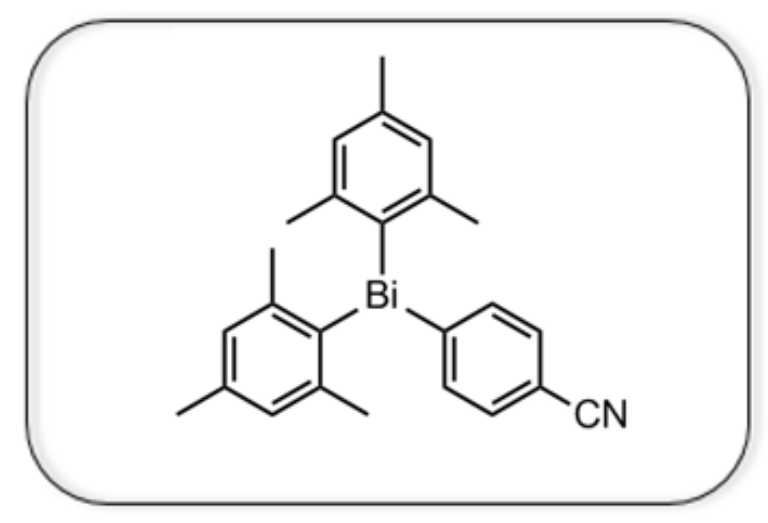

Dimesityl(4-cyanophenyl)bismuthane (1g): Yield: $92 \%$. This compound was obtained according to procedure $\mathbf{B}$ from (4-cyanophenyl)zinc(II) halide $(1.123 \mathrm{mmol})$ and dimesitylbismuth iodide $(0.537 \mathrm{~g}$, $0.936 \mathrm{mmol})$. Recrystallized from hot ethanol.

White solid. ${ }^{1} \mathbf{H}$ NMR (CDCl $): \delta 8.03\left(\mathrm{~d}, J=8.0 \mathrm{~Hz}, 2 \mathrm{H}, \mathrm{C}_{6} H_{4} \mathrm{CN}\right), 7.51\left(\mathrm{~d}, J=8.0 \mathrm{~Hz}, 2 \mathrm{H}, \mathrm{C}_{6} H_{4} \mathrm{CN}\right)$, $7.01\left(\mathrm{~s}, 4 \mathrm{H}, \mathrm{C}_{6} H_{2}\right), 2.27\left(\mathrm{~s}, 6 \mathrm{H}, \mathrm{C}_{6} \mathrm{H}_{2}-p \mathrm{CH}_{3}\right), 2.22\left(\mathrm{~s}, 12 \mathrm{H}, \mathrm{C}_{6} \mathrm{H}_{2}-o \mathrm{CH}_{3}\right) .{ }^{13} \mathbf{C ~ N M R}\left(\mathbf{C D C l}_{3}\right): \delta 156.9,156.6$, 145.3, $139.3\left(\mathrm{C}_{6} \mathrm{H}_{4} \mathrm{CN}, \mathrm{CH}\right), 138.0,132.5\left(\mathrm{C}_{6} \mathrm{H}_{4} \mathrm{CN}, \mathrm{CH}\right), 129.6\left(\mathrm{C}_{6} \mathrm{H}_{2}, \mathrm{CH}\right)$, 119.2, 110.7, $27.8\left(\mathrm{C}_{6} \mathrm{H}_{2}-\right.$ $\left.o \mathrm{CH}_{3}\right), 21.0\left(\mathrm{C}_{6} \mathrm{H}_{2}-p C \mathrm{H}_{3}\right)$. Anal. Calc. for $\mathbf{B i C}_{25} \mathbf{H}_{26} \mathbf{N}$ : C, 54.65; H, 4.77; N, 2.35. Found: C, 54.83; H, $4.88 ; \mathrm{N}, 2.40$.

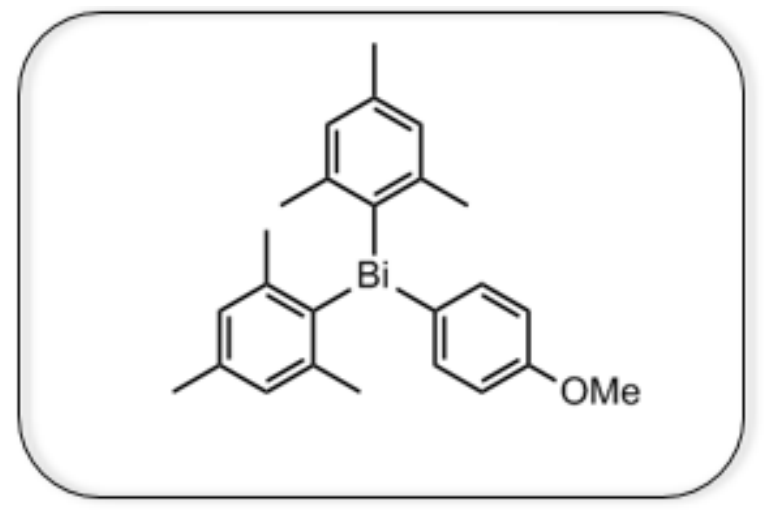

\section{Dimesityl(4-methoxyphenylbismuthane}

(1h):

Yield: 94\%. This compound was obtained according to procedure B from (4-methoxyphenyl) magnesium(II) bromide $(1.123 \mathrm{mmol})$ and dimesitylbismuth iodide $(0.537 \mathrm{~g}, 0.936 \mathrm{mmol})$. Recrystallized from cold hexanes.

White solid. ${ }^{1} \mathbf{H}$ NMR $\left(\mathbf{C D C l}_{3}\right): \delta 7.82\left(\mathrm{~d}, J=8.5 \mathrm{~Hz}, 2 \mathrm{H}, \mathrm{C}_{6} H_{4}-\mathrm{OCH}_{3}\right), 6.98\left(\mathrm{~s}, 4 \mathrm{H}, \mathrm{C}_{6} H_{2}\right), 6.87(\mathrm{~d}, J=$ $\left.8.5 \mathrm{~Hz}, 2 \mathrm{H}, \mathrm{C}_{6} \mathrm{H}_{4}-\mathrm{OCH}_{3}\right), 3.79\left(\mathrm{~s}, 3 \mathrm{H}, \mathrm{C}_{6} \mathrm{H}_{4}-\mathrm{OCH}_{3}\right), 2.27$ (overlapping singlets, $\left.18 \mathrm{H}, \mathrm{C}_{6} \mathrm{H}_{2}-\mathrm{CH}_{3}\right) .{ }^{13} \mathbf{C} \mathbf{~ N M R}$ $\left(\mathbf{C D C l}_{3}\right): \delta 158.9,155.5,145.5,140.6,139.9\left(\mathrm{C}_{6} \mathrm{H}_{4}-\mathrm{OCH}_{3}, \mathrm{CH}\right), 137.4,129.1\left(\mathrm{C}_{6} \mathrm{H}_{2}, \mathrm{CH}\right), 115.8\left(\mathrm{C}_{6} \mathrm{H}_{4}-\right.$ $\left.\mathrm{OCH}_{3}, \mathrm{CH}\right), 54.9\left(\mathrm{C}_{6} \mathrm{H}_{4}-\mathrm{OCH}_{3}\right), 27.8\left(\mathrm{C}_{6} \mathrm{H}_{2}-\mathrm{CH}_{3}\right), 21.0\left(\mathrm{C}_{6} \mathrm{H}_{2}-\mathrm{CH}_{3}\right)$. Anal. Calc. for $\mathbf{B i O C}_{25} \mathbf{H}_{29}$ : C, 54.15; $\mathrm{H}, 5.27$. Found: C, 54.10; H, 5.15. 


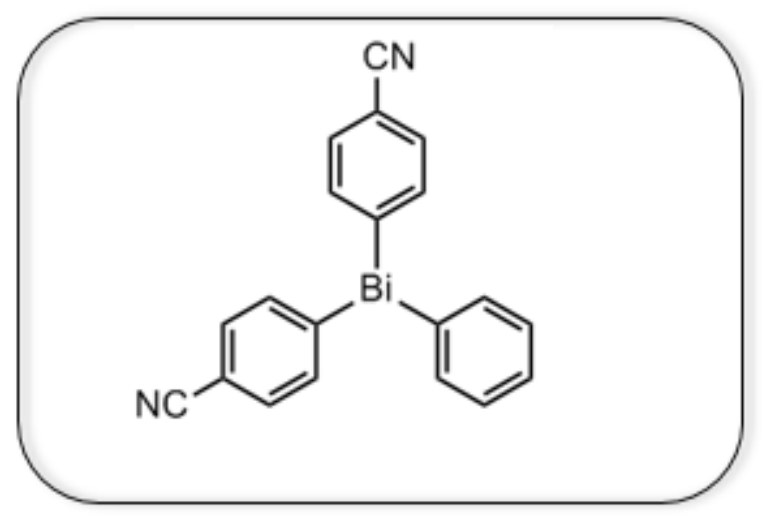

Di(4-cyanophenyl)phenylbismuthane (1i): Yield: $87 \%$. This compound was obtained according to procedure $\mathbf{C}$ from (4-cyanophenyl)zinc(II) halide (2.06 mmol) and phenylbismuth ditosylate $(0.587 \mathrm{~g}, 0.936$ mmol). Recrystallized from toluene/hexanes.

White solid. ${ }^{1} \mathbf{H}$ NMR (CDCl $): \delta 7.84\left(\mathrm{~d}, J=8.0 \mathrm{~Hz}, 4 \mathrm{H}, \mathrm{C}_{6} H_{4} \mathrm{CN}\right), 7.69\left(\mathrm{~d}, J=6.5 \mathrm{~Hz}, 2 \mathrm{H}, \mathrm{C}_{6} H_{5}\right), 7.64$ $\left(\mathrm{d}, J=8.0 \mathrm{~Hz}, 4 \mathrm{H}, \mathrm{C}_{6} H_{4} \mathrm{CN}\right), 7.49-7.46\left(\mathrm{~m}, 2 \mathrm{H}, \mathrm{C}_{6} H_{5}\right), 7.43-7.37,\left(\mathrm{~m}, 1 \mathrm{H}, \mathrm{C}_{6} H_{5}\right) .{ }^{13} \mathbf{C} \mathbf{N M R}\left(\mathbf{C D C l}_{3}\right): \delta$ 161.8, 156.3, $138.0\left(\mathrm{C}_{6} \mathrm{H}_{4} \mathrm{CN}, \mathrm{CH}\right), 137.3(\mathrm{Ph}, \mathrm{CH}), 133.5\left(\mathrm{C}_{6} \mathrm{H}_{4} \mathrm{CN}, \mathrm{CH}\right), 131.2(\mathrm{Ph}, \mathrm{CH}), 128.6(\mathrm{Ph}, \mathrm{CH})$, 118.8, 111.9. Anal. Calc. for $\mathbf{B i N}_{\mathbf{2}} \mathbf{C}_{\mathbf{2}} \mathbf{H}_{13}$ : C, 48.99; H, 2.67; N, 5.71. Found: C, 49.15; H, 2.62; N, 5.87.

The synthesis of this compound was attempted through procedure A utilizing phenyl magnesium bromide and bis(4-cyanophenyl)bismuth tosylate. The yield for this reaction was only $45 \%$, as the corresponding tosylate was not obtained in analytical purity.

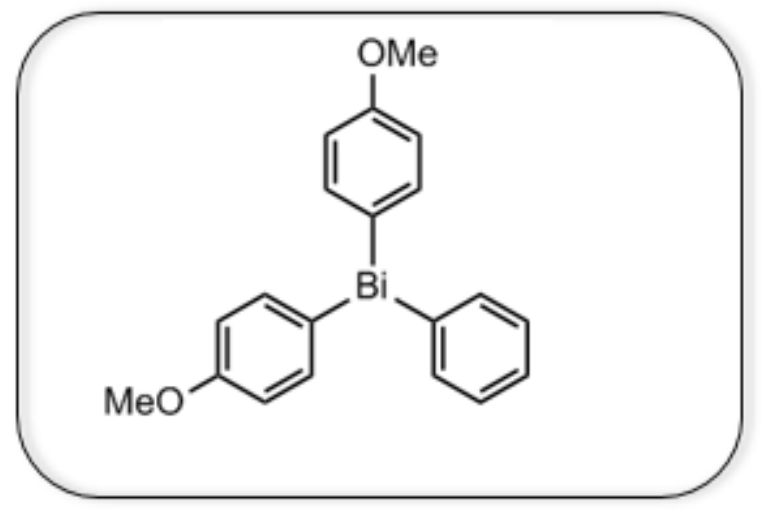

Di(4-methoxyphenyl)phenylbismuthane $\quad(1 \mathbf{j})$ : Yield: 91\%. This compound was obtained according to procedure C from (4methoxyphenyl)magnesium(II) bromide (2.06 mmol) and phenylbismuth ditosylate $(0.587 \mathrm{~g}, 0.936 \mathrm{mmol})$. Purified by column chromatography and eluted with a gradient mixture of solvents (hexane/ethyl acetate $100 / 0$ to $25 / 75$.

White solid. ${ }^{1} \mathbf{H}$ NMR $\left(\mathbf{C D C l}_{3}\right): \delta 7.74\left(\mathrm{~d}, J=7.5,2 \mathrm{H}, \mathrm{C}_{6} H_{5}\right), 7.64\left(\mathrm{~d}, J=8.6,4 \mathrm{H}, \mathrm{C}_{6} H_{4}-\mathrm{OCH}_{3}\right), 7.40-7.37$ $\left(\mathrm{m}, J=7.5,2 \mathrm{H}, \mathrm{C}_{6} H_{5}\right), 7.34-7.31,\left(\mathrm{~m}, 1 \mathrm{H}, \mathrm{C}_{6} H_{5}\right), 6.94,\left(\mathrm{~d}, J=8.6,4 \mathrm{H}, \mathrm{C}_{6} H_{4}-\mathrm{OCH}_{3}\right), 3.80\left(\mathrm{~s}, 6 \mathrm{H}, \mathrm{C}_{6} \mathrm{H}_{4}-\right.$ $\left.\mathrm{OCH}_{3}\right) .{ }^{13} \mathbf{C}$ NMR $\left(\mathbf{C D C l}_{3}\right): 159.3,154.6,145.3,138.8\left(\mathrm{C}_{6} \mathrm{H}_{4}-\mathrm{OCH}_{3}, \mathrm{CH}\right), 137.4(\mathrm{Ph}, \mathrm{CH}), 130.3(\mathrm{Ph}$, $\mathrm{CH})$, $127.6(\mathrm{Ph}, \mathrm{CH}), 116.3\left(\mathrm{C}_{6} \mathrm{H}_{4}-\mathrm{OCH}_{3}, \mathrm{CH}\right), 55.0\left(\mathrm{C}_{6} \mathrm{H}_{4}-\mathrm{OCH}_{3}\right)$. Anal. Calc. for $\mathbf{B i O}_{2} \mathbf{C}_{20} \mathbf{H}_{19}$ : C, 48.01; $\mathrm{H}, 3.83$. Found: $\mathrm{C}, 48.24 ; \mathrm{H}, 3.66$.

The synthesis of this compound was attempted through procedure A utilizing phenyl magnesium bromide and bis(4-methoxyphenyl)bismuth tosylate. The yield for this reaction was $87 \%$, however the corresponding tosylate was not obtained in analytical purity. 


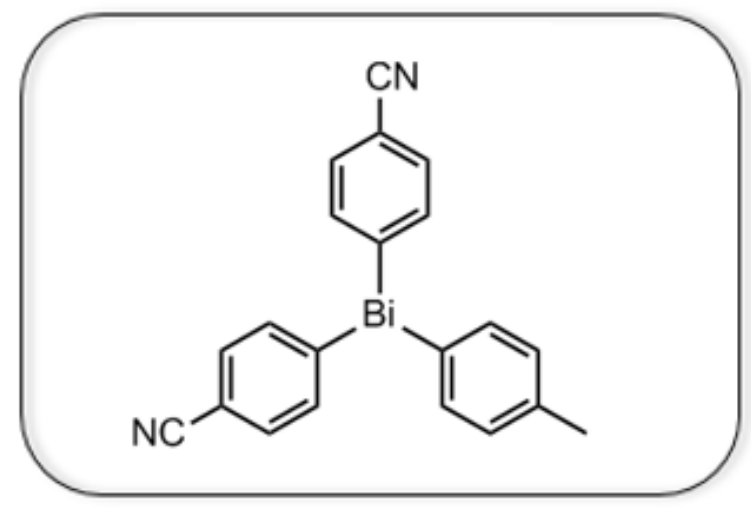

Di(4-cyanophenyl)(p-tolyl)bismuthane (1k): Yield: $87 \%$. This compound was obtained according to procedure $\mathbf{C}$ from (4-cyanophenyl)zinc(II) iodide $(2.06 \mathrm{mmol})$ and ( $p$-tolyl)bismuth ditosylate $(0.547$ g, $0.936 \mathrm{mmol})$. Purified by column chromatography and eluted with a gradient mixture of solvents (hexane/ethyl acetate $100 / 0$ to $25 / 75$.

White solid. ${ }^{1} \mathbf{H}$ NMR (CDCl 3 ): $\delta 7.84\left(\mathrm{~d}, J=8.2 \mathrm{~Hz}, 4 \mathrm{H}, \mathrm{C}_{6} H_{4} \mathrm{CN}\right), 7.63\left(\mathrm{~d}, J=8.2 \mathrm{~Hz}, 4 \mathrm{H}, \mathrm{C}_{6} H_{4} \mathrm{CN}\right)$, $7.57\left(\mathrm{~d}, J=7.8 \mathrm{~Hz}, 2 \mathrm{H}, \mathrm{C}_{6} H_{4}-p \mathrm{CH}_{3}\right), 7.29\left(\mathrm{~d}, J=7.8 \mathrm{~Hz}, 2 \mathrm{H}, \mathrm{C}_{6} H_{4}-p \mathrm{CH}_{3}\right), 2.35\left(\mathrm{~s}, 3 \mathrm{H}, \mathrm{C}_{6} \mathrm{H}_{4}-\mathrm{CH}_{3}\right) .{ }^{13} \mathbf{C}$ NMR $\left(\mathbf{C D C l}_{3}\right): \delta 161.6,152.8,138.6,138.0\left(\mathrm{C}_{6} \mathrm{H}_{4} \mathrm{CN}, C \mathrm{H}\right), 137.3\left(\mathrm{C}_{6} \mathrm{H}_{4}-p \mathrm{CH}_{3}, C \mathrm{H}\right), 133.5\left(\mathrm{C}_{6} \mathrm{H}_{4} \mathrm{CN}\right.$, $C \mathrm{H}), 132.0\left(\mathrm{C}_{6} \mathrm{H}_{4}-p \mathrm{CH}_{3}, C \mathrm{H}\right), 118.8,111.7,21.5\left(\mathrm{C}_{6} \mathrm{H}_{4}-p C \mathrm{H}_{3}\right)$. Anal. Calc. for $\mathbf{B i N}_{2} \mathbf{C}_{21} \mathbf{H}_{15}: \mathrm{C}, 50.01 ; \mathrm{H}$, $3.00 ; \mathrm{N}, 5.55$. Found: C, 50.01; H, 2.87; N, 5.46.

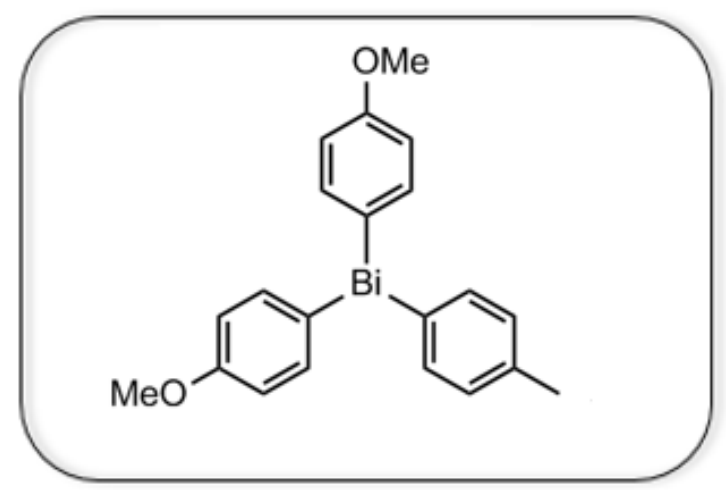

Di(4-methoxyphenyl)(p-tolyl)bismuthane (11): Yield: $52 \%$. This compound was obtained according to procedure $\mathbf{C}$ from (4-methoxyphenyl)magnesium(II) bromide $(2.06 \mathrm{mmol})$ and ( $p$-tolyl) bismuth ditosylate (0.547 g, $0.936 \mathrm{mmol})$. Purified by column chromatography and eluted with a gradient mixture of solvents (hexane/ethyl acetate $100 / 0$ to $20 / 80$.

White solid. ${ }^{1} \mathbf{H}$ NMR (CDCl $)$ ): $\delta$ 7.65-7.61 (m, 6H, Ar), $7.20\left(\mathrm{~d}, J=7.5 \mathrm{~Hz}, 2 \mathrm{H}, \mathrm{C}_{6} H_{4}-p \mathrm{CH}_{3}\right), 6.93(\mathrm{~d}, J$ $\left.=8.4 \mathrm{~Hz}, 4 \mathrm{H}, \mathrm{C}_{6} \mathrm{H}_{4}-\mathrm{OCH}_{3}\right), 3.80\left(\mathrm{~s}, 6 \mathrm{H}, \mathrm{C}_{6} \mathrm{H}_{4}-\mathrm{OCH}_{3}\right), 2.34\left(\mathrm{~s}, 3 \mathrm{H}, \mathrm{C}_{6} \mathrm{H}_{4}-\mathrm{CH}_{3}\right) .{ }^{13} \mathbf{C} \mathbf{N M R}\left(\mathbf{C D C l}_{3}\right): \delta 159.2$, 150.9, 145.1, $138.7\left(\mathrm{C}_{6} \mathrm{H}_{4}-\mathrm{OCH}_{3}, C \mathrm{H}\right), 137.4\left(\mathrm{C}_{6} \mathrm{H}_{4}-p \mathrm{CH}_{3}, C \mathrm{H}\right), 137.3,131.2\left(\mathrm{C}_{6} \mathrm{H}_{4}-p \mathrm{CH}_{3}, C \mathrm{H}\right), 116.3$ $\left(\mathrm{C}_{6} \mathrm{H}_{4}-\mathrm{OCH}_{3}, \mathrm{CH}\right), 55.0\left(\mathrm{C}_{6} \mathrm{H}_{4}-\mathrm{OCH}_{3}\right), 21.5\left(\mathrm{C}_{6} \mathrm{H}_{4}-\mathrm{CH}_{3}\right)$. Anal. Calc. for $\mathbf{B i O}_{2} \mathbf{C}_{21} \mathbf{H}_{21}: \mathrm{C}, 49.04 ; \mathrm{H}, 4.12$. Found: C, 49.06; H, 3.90 
6. EA Report and NMR Spectra for $3 b$

\section{NMR Spectra and EA Report of Compound 3b}<smiles>Cc1ccc([BH2-]OS(=O)(=O)c2ccc(C)cc2)cc1</smiles>

Chemical Formula: $\mathrm{BiO}_{6} \mathrm{C}_{21} \mathrm{H}_{21} \mathrm{~S}_{2}$

Molecular Weight: 642.49

Elemental Analysis: C: 39.26; Bi: 32.53; H: 3.29; O: 14.94; S: 9.98

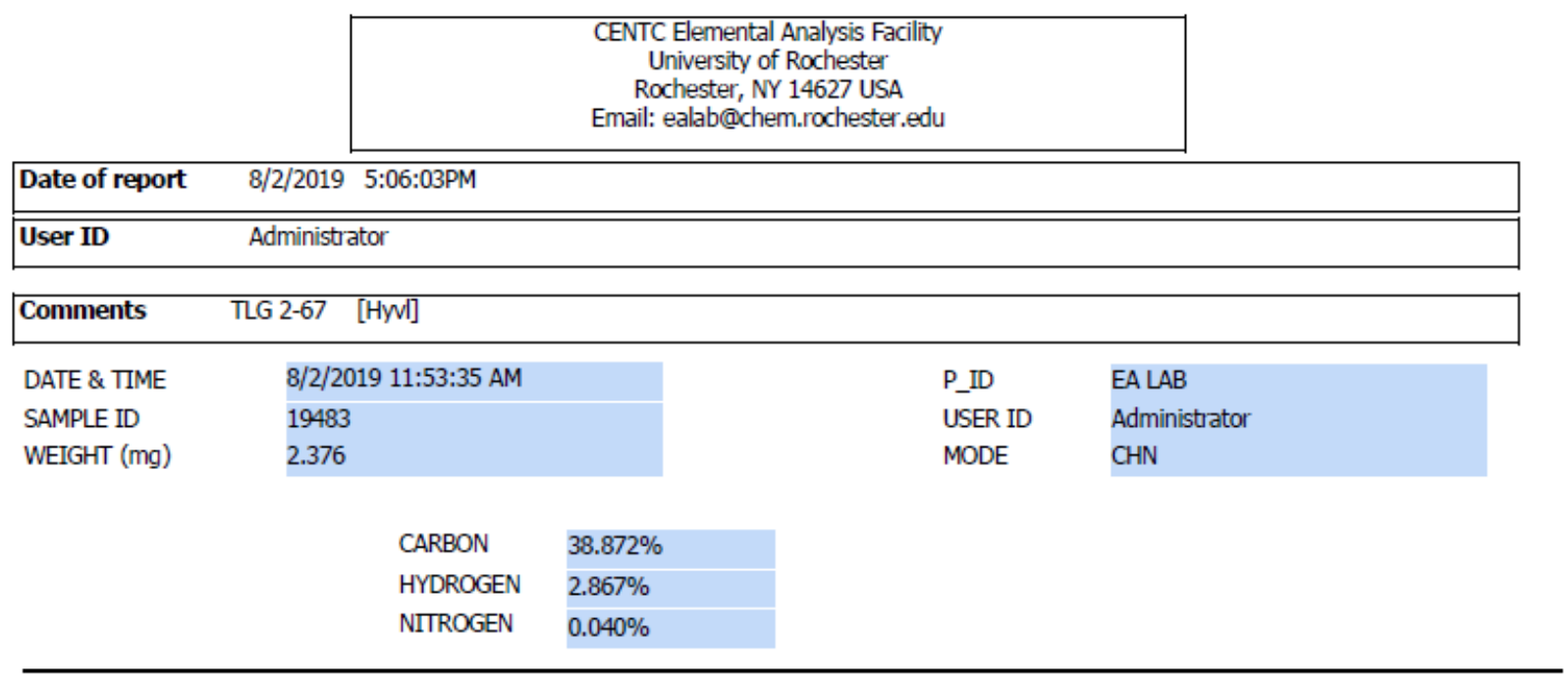

Acknowledgment

Analytical data were obtained from the CENTC Elemental Analysis Facility at the University of Rochester, funded by NSF CHE-0650456.

Instrumentation

Microanalysis samples were weighed with a PerkinElmer Model AD6000 Autobalance and their compositions were determined with a PerkinElmer 2400 Series II Analyzer.

Figure S1. EA Report of 3b 


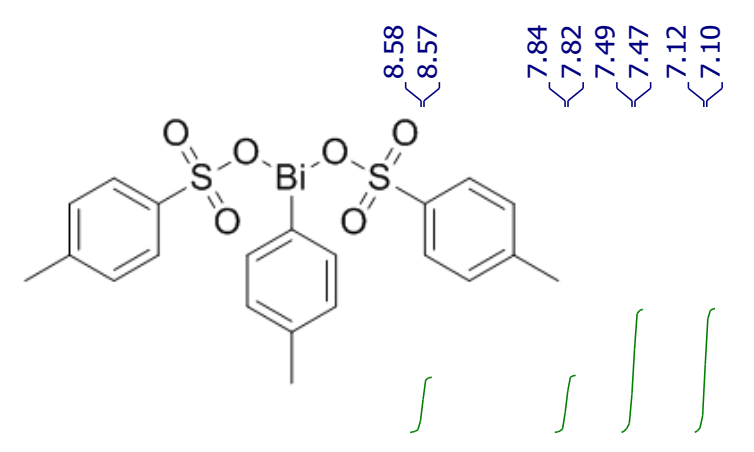

$\stackrel{\infty}{\stackrel{\infty}{N}}$

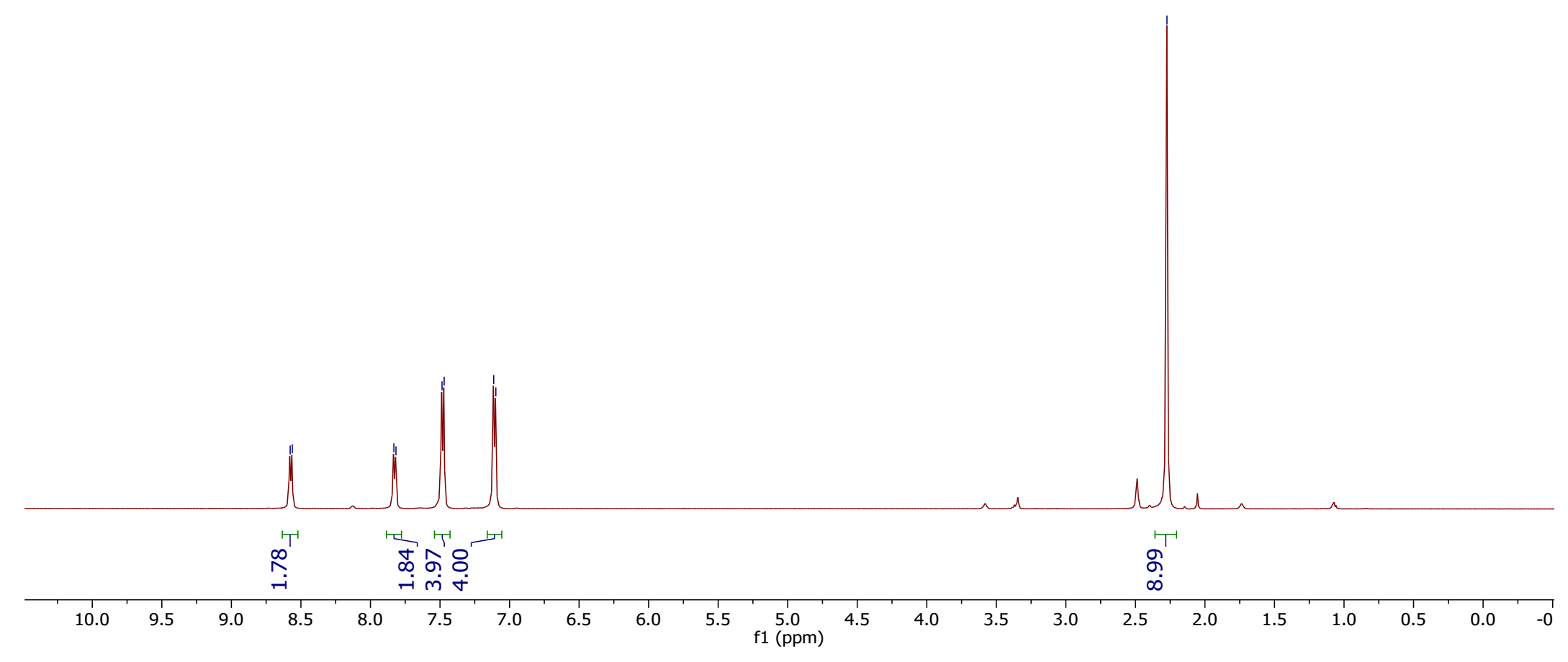

Figure S2. ${ }^{1} \mathrm{HNMR}$ of $\mathbf{3 b}$ in $\mathrm{d}_{6}$-DMSO 


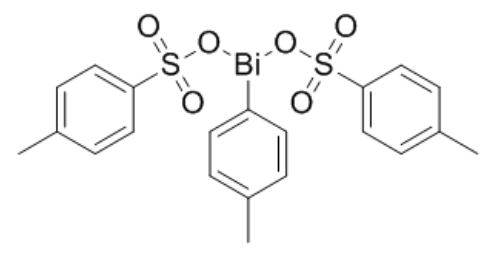

$\underbrace{-10}$
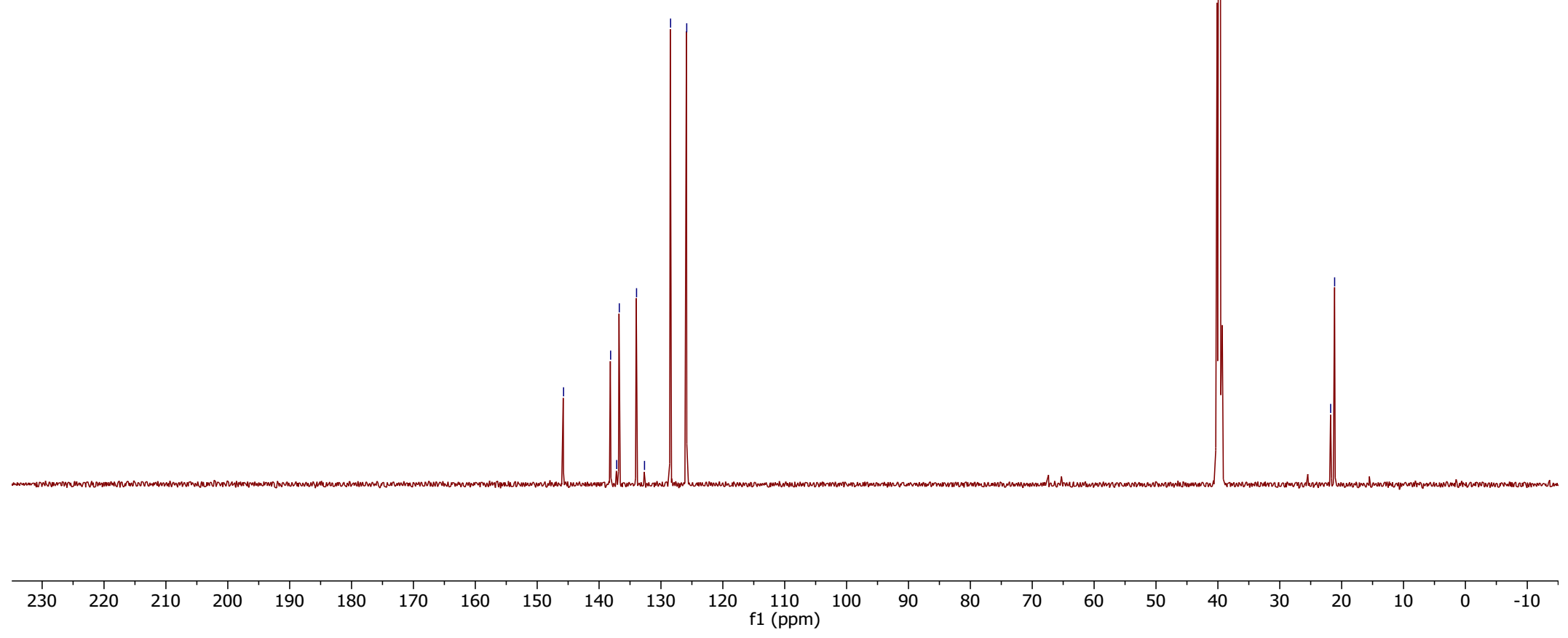

Figure S3. ${ }^{13} \mathrm{C}\{\mathrm{H}\}$ NMR of $\mathbf{3 b}$ in $\mathrm{d}_{6}-\mathrm{DMSO}$

S15 

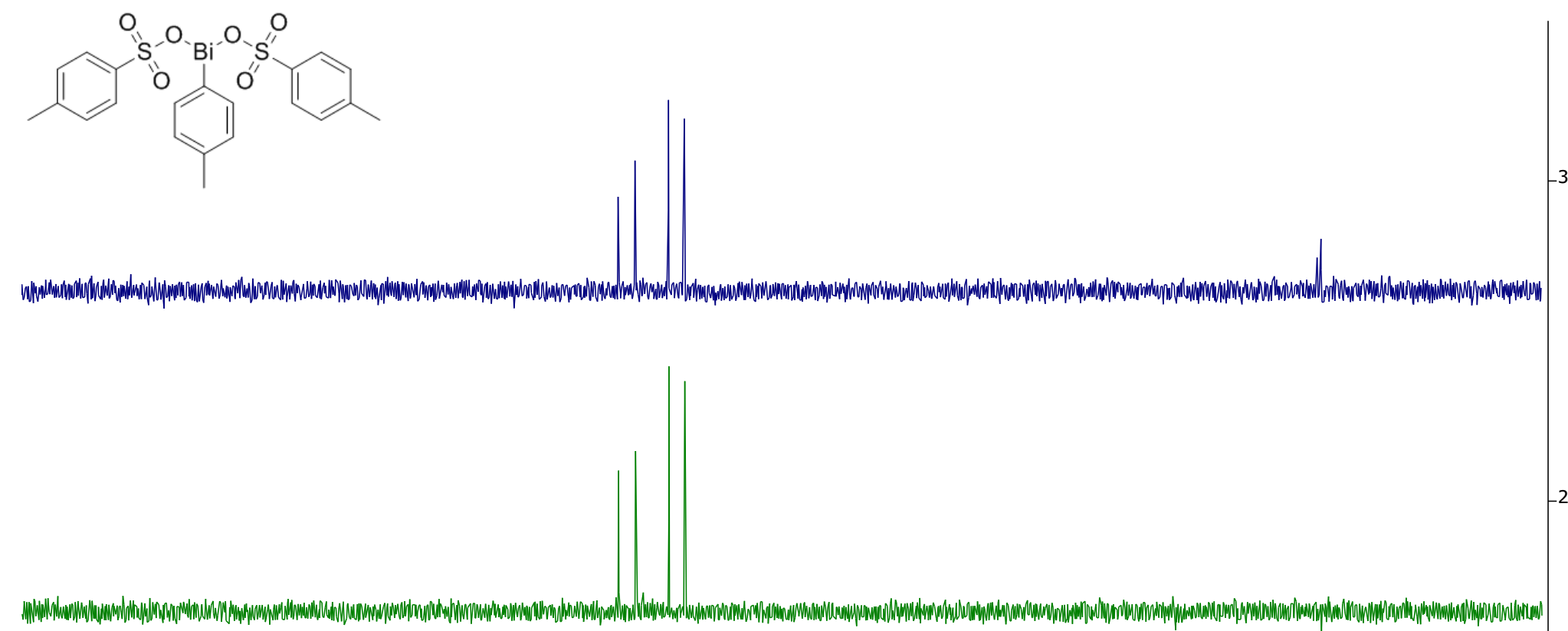

4.

$\begin{array}{lllllllllllllllllllllllllllllllllllll}230 & 220 & 210 & 200 & 190 & 180 & 170 & 160 & 150 & 140 & 130 & 120 & 110 & 100 & 90 & 80 & 70 & 60 & 50 & 40 & 30 & 20 & 10 & 0 & -10\end{array}$

Figure S4. ${ }^{13} \mathrm{C}$ NMR DEPT of $\mathbf{3} \mathbf{b}$ in $\mathrm{d}_{6}$-DMSO 


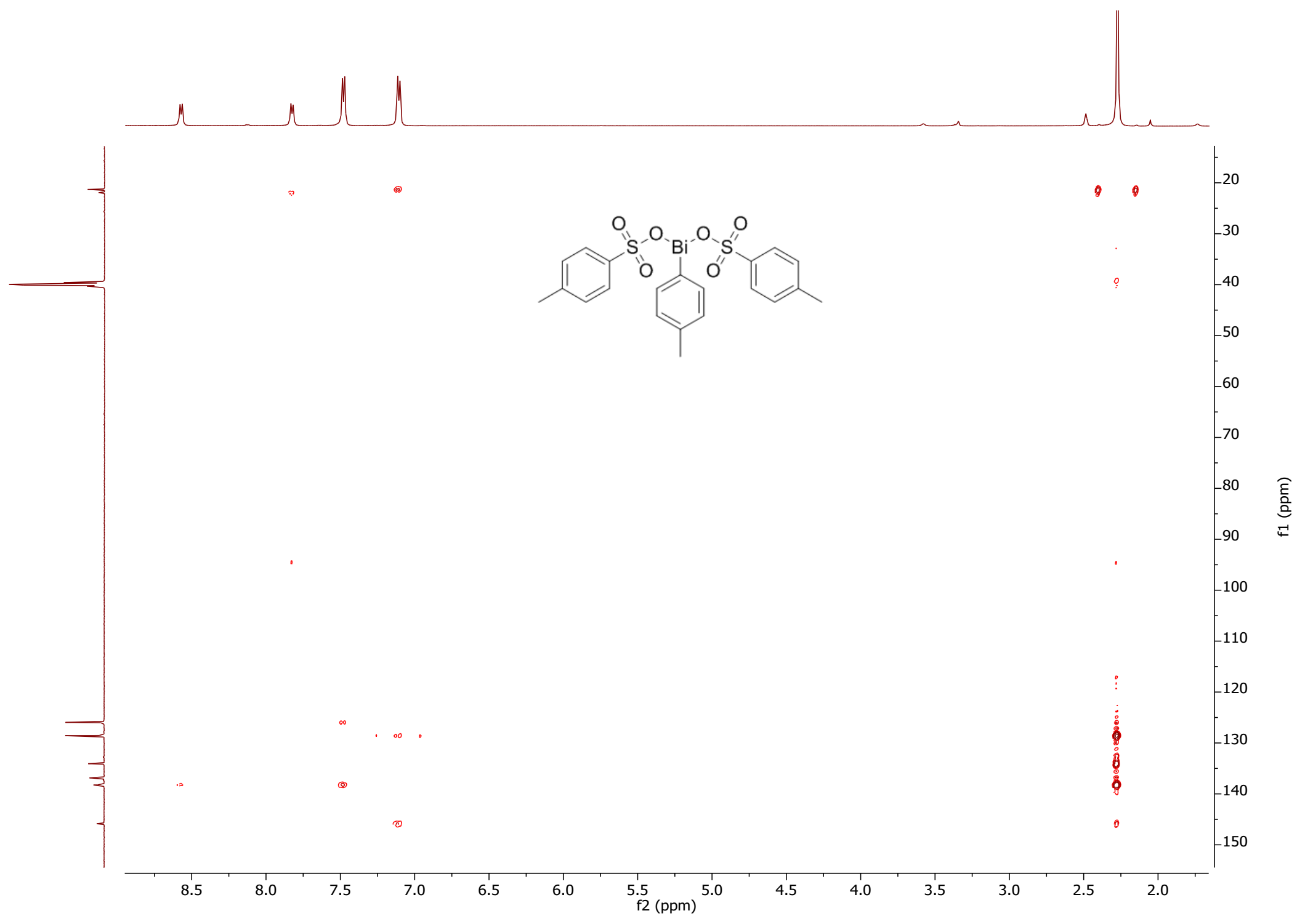

Figure S5. 2D HSQC of $\mathbf{3 b}$ in $\mathrm{d}_{6}$-DMSO 


\section{EA Report and NMR Spectra for Compounds 1}

\section{NMR Spectra and EA Report of Compound 1a}<smiles>COC(OC)c1ccc([Bi](c2ccccc2)c2ccccc2)cc1</smiles>

Chemical Formula: $\mathrm{BiO}_{2} \mathrm{C}_{21} \mathrm{H}_{21}$

Molecular Weight: 514.38

Elemental Analysis: C: 49.04; Bi: 40.63; H: 4.12; O: 6.22

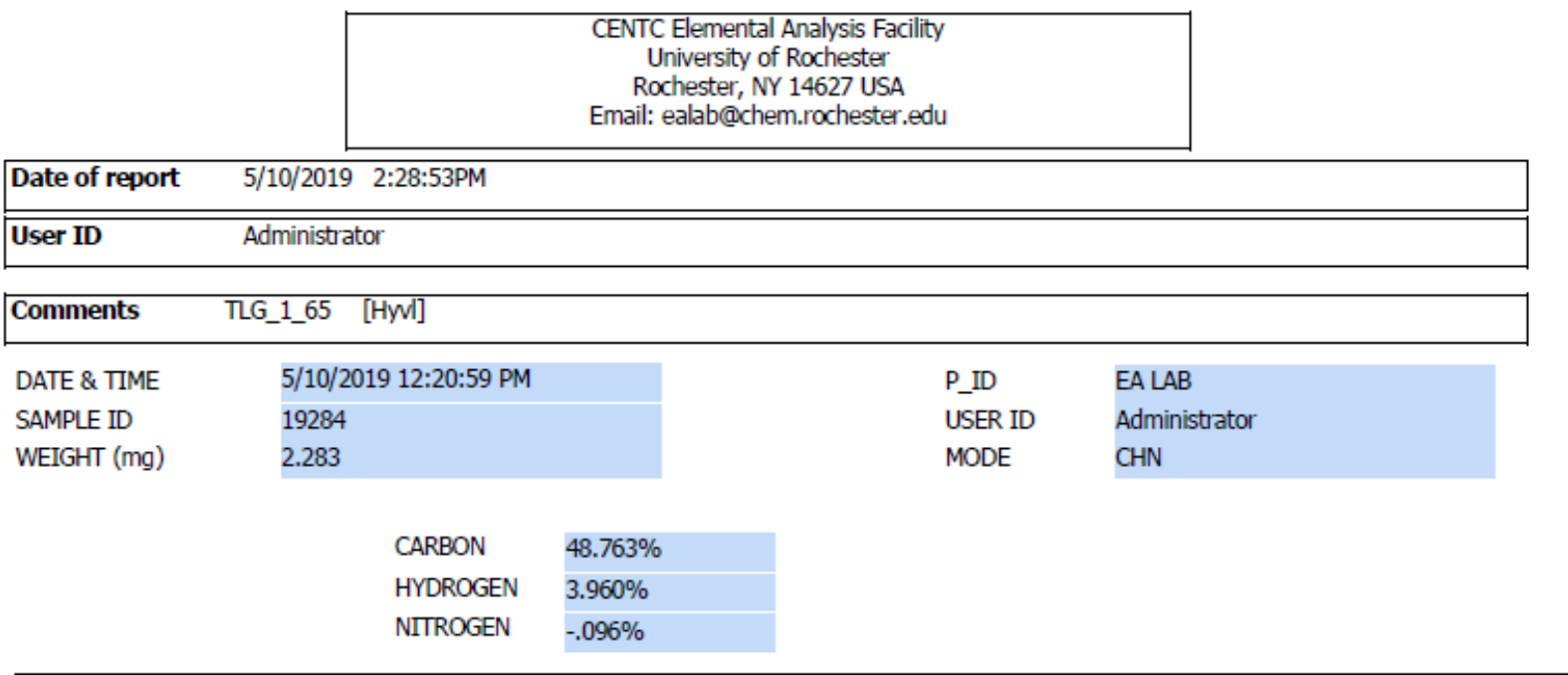

Acknowledgment

Analytical data were obtained from the CENTC Elemental Analysis Facility at the University of Rochester, funded by NSF CHE-0650456.

\section{Instrumentation}

Microanalysis samples were weighed with a PerkinElmer Model AD6000 Autobalance and their compositions were determined with a PerkinElmer 2400 Series II Analyzer.

Figure S6. EA Report of 1a 

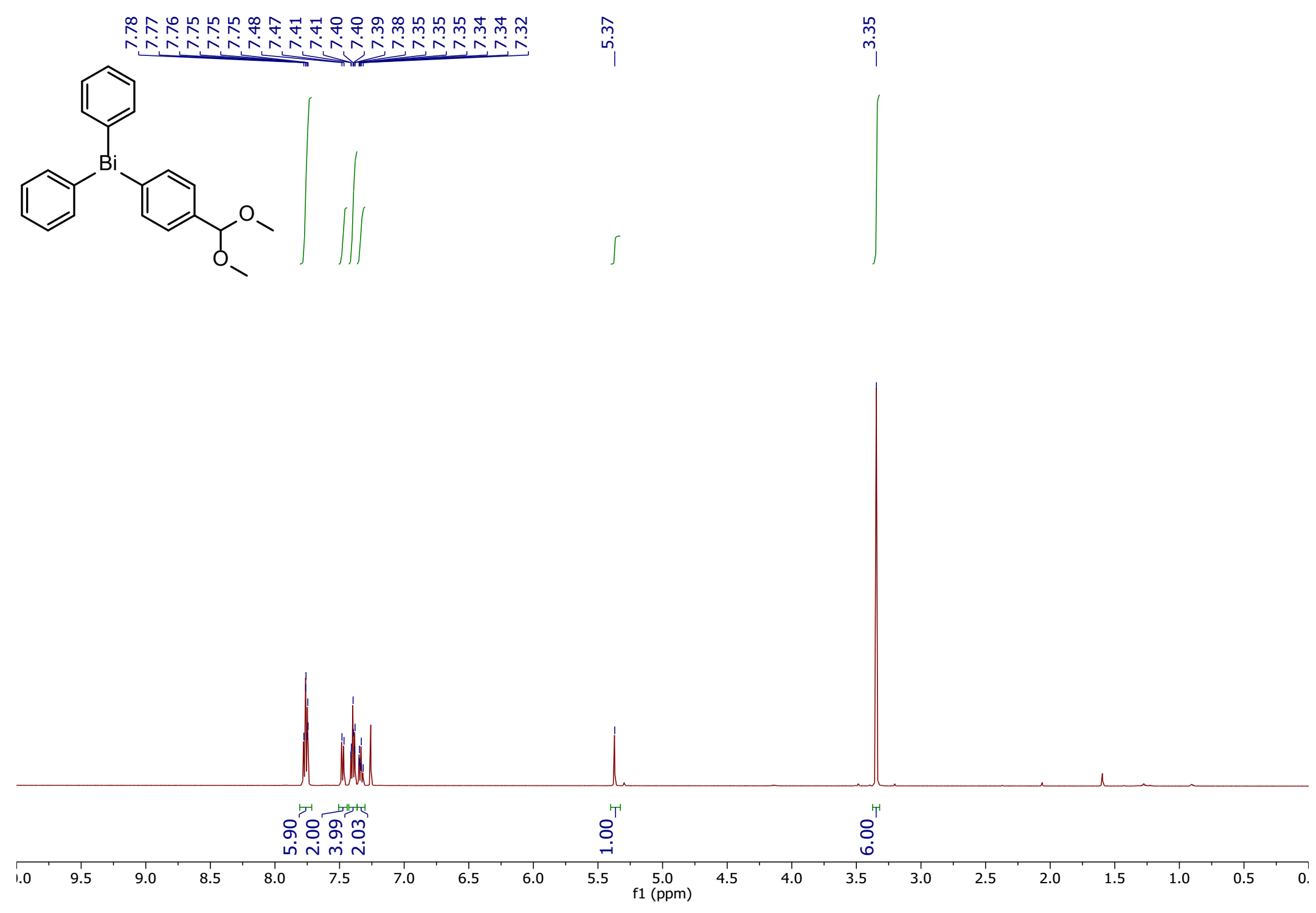

Figure S7. ${ }^{1} \mathrm{HNMR}$ of $\mathbf{1 a}$ in $\mathrm{CDCl}_{3}$ 


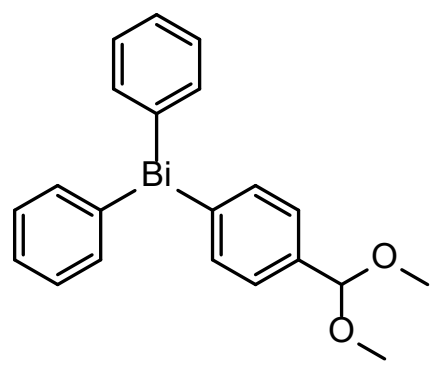

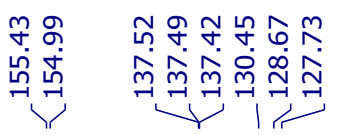

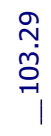

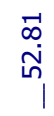

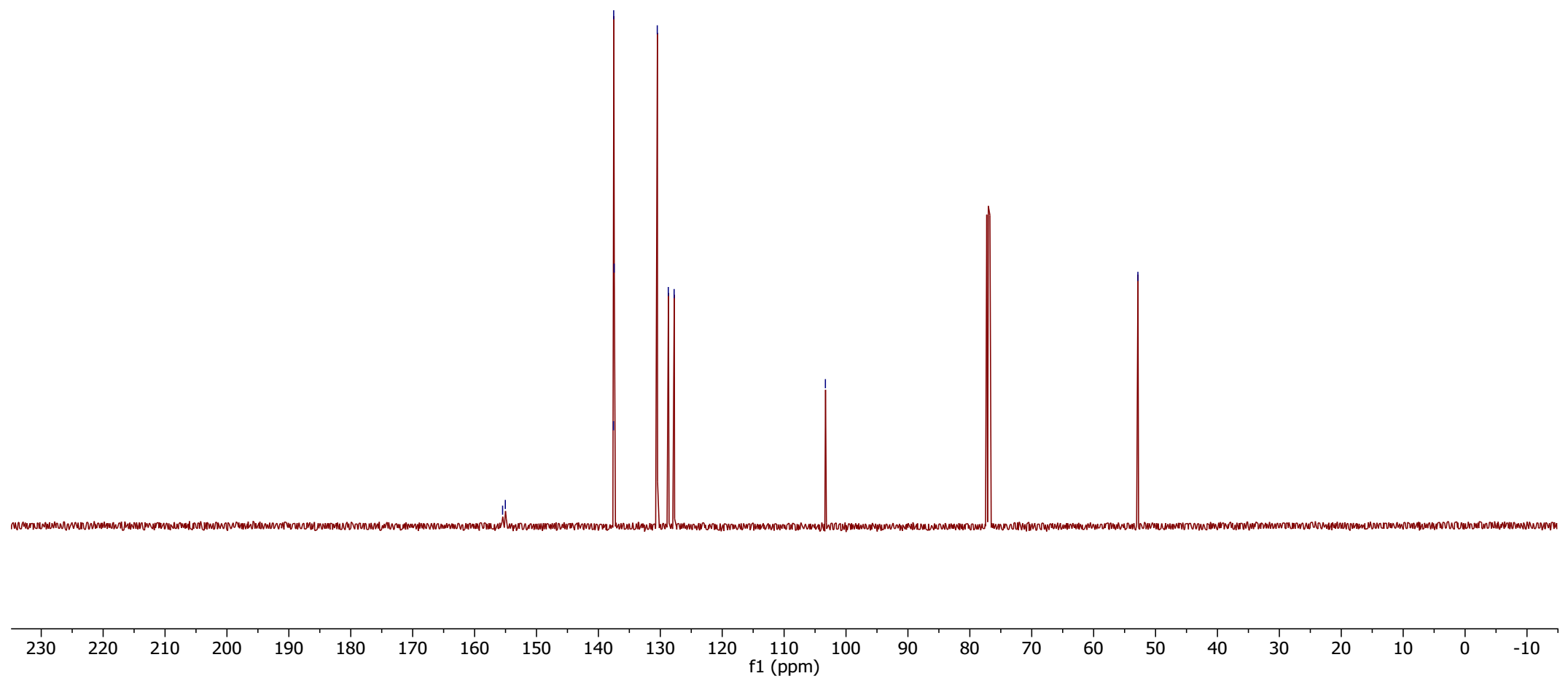

Figure S8. ${ }^{13} \mathrm{C}\left\{{ }^{1} \mathrm{H}\right\}$ NMR of $\mathbf{1 a}$ in $\mathrm{CDCl}_{3}$

S20 
<smiles>COC(OC)c1ccc([Bi](c2ccccc2)c2ccccc2)cc1</smiles>

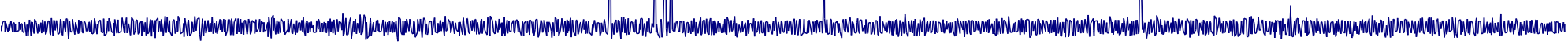
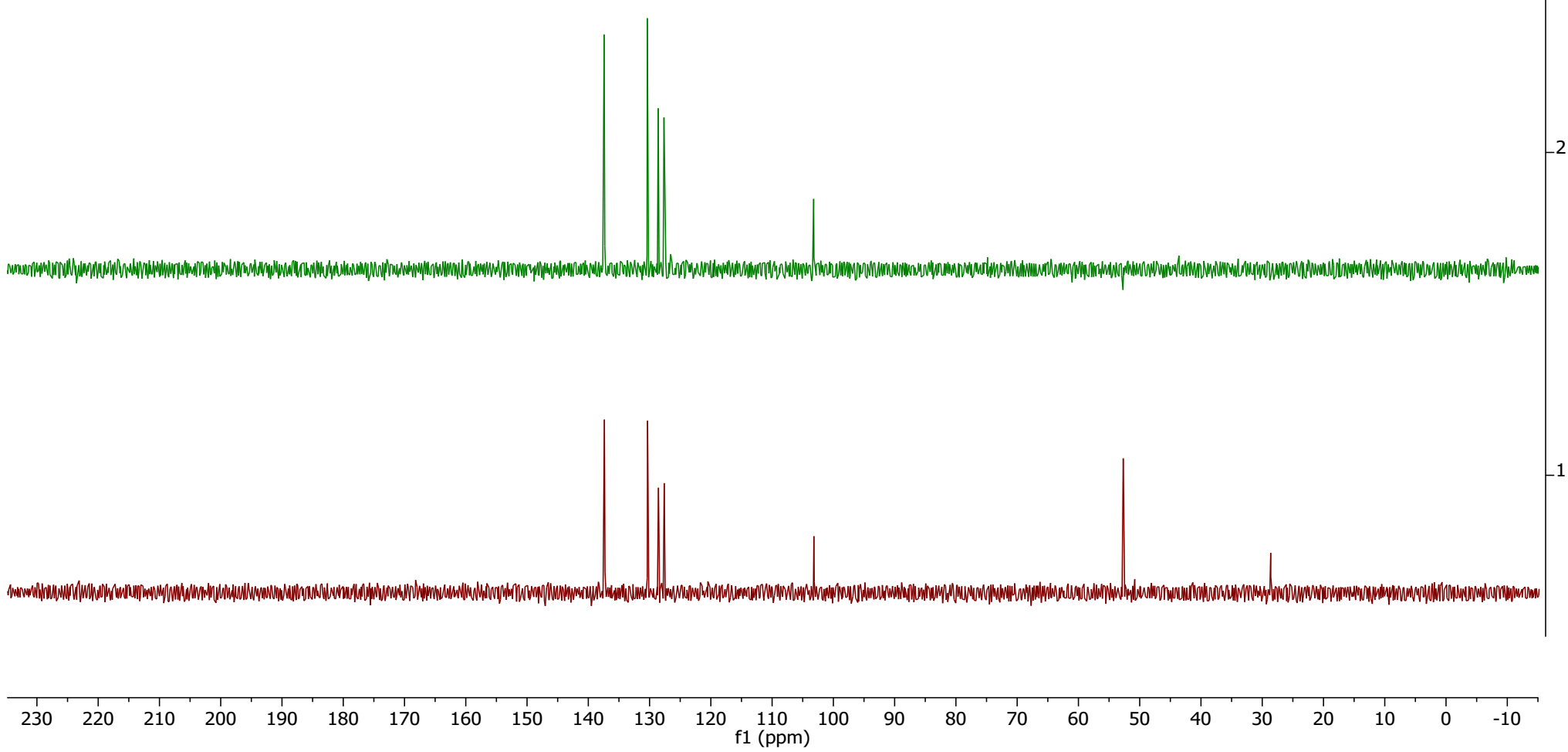

Figure S9. ${ }^{13} \mathrm{C}$ DEPT of $\mathbf{1 a}$ in $\mathrm{CDCl}_{3}$

S21 


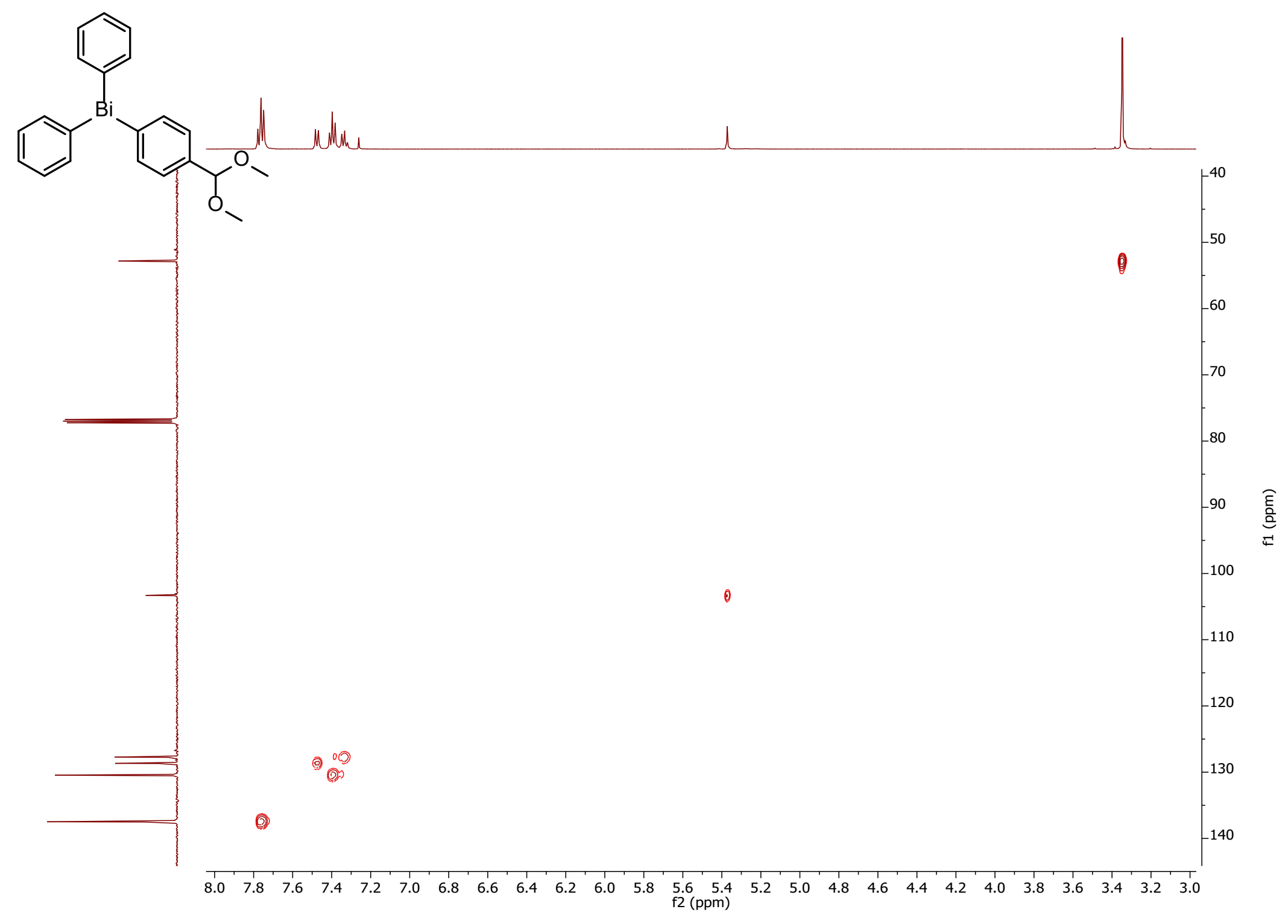

Figure S10. 2D HSQC of $1 \mathrm{a}$ in $\mathrm{CDCl}_{3}$ 


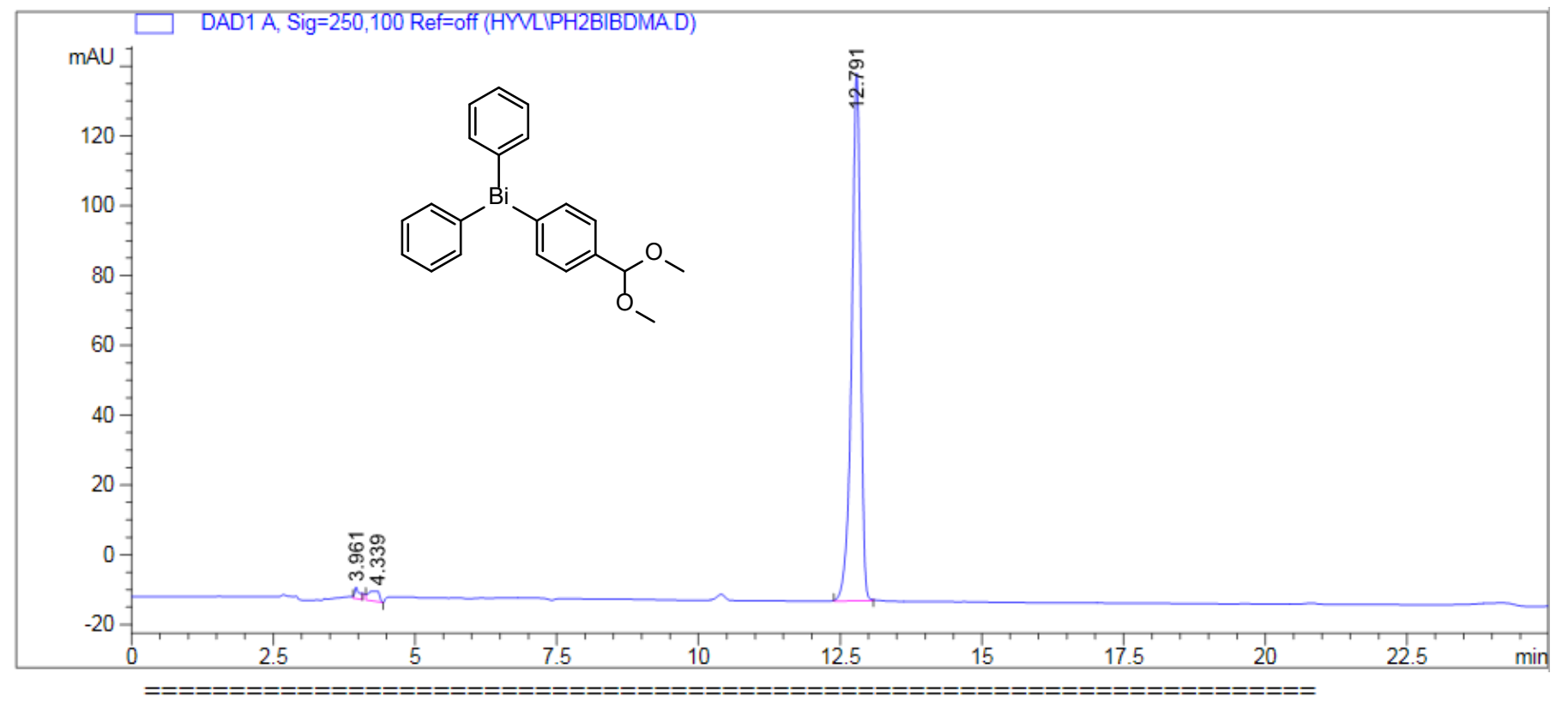

Area Percent Report

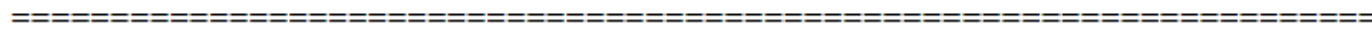

$\begin{array}{lccc}\text { Sorted By } & : & \text { Signal } \\ \text { Multiplier: } & : & 1.0000 & \\ \text { Dilution: } & : & 1.0000 & \\ \text { Sample Amount: } & : & 20.00000 \text { [ng/ul] } & \text { (not used in calc.) }\end{array}$

Use Multiplier \& Dilution Factor with ISTDs

Signal 1: DAD1 A, Sig=250,100 Ref=off

\begin{tabular}{|c|c|c|c|c|c|c|}
\hline $\begin{array}{c}\text { Peak } \\
\#\end{array}$ & $\begin{array}{c}\text { RetTime } \\
\text { [min] }\end{array}$ & Type & $\begin{array}{c}\text { Width } \\
\text { [min] }\end{array}$ & $\begin{array}{c}\text { Area } \\
{\left[\mathrm{mAU}^{\star} s\right]}\end{array}$ & $\begin{array}{l}\text { Height } \\
\text { [mAU] }\end{array}$ & $\begin{array}{c}\text { Area } \\
\frac{8}{\delta}\end{array}$ \\
\hline-- & -1 & & -1 & ------- & -------1 & ------ \\
\hline 1 & 3.961 & $\mathrm{BB}$ & 0.0811 & 19.33534 & 3.26655 & 1.1250 \\
\hline 2 & 4.339 & BV & 0.1627 & 38.95380 & 3.05713 & 2.2664 \\
\hline 3 & 12.791 & $\mathrm{BB}$ & 0.1660 & 1660.45557 & 151.28766 & 96.6086 \\
\hline
\end{tabular}

Figure S11. HPLC Chromatogram of 1a 


\section{NMR Spectra and EA Report of Compound 1b}<smiles>COc1ccc([Bi](c2ccccc2)c2ccccc2)cc1</smiles>

Chemical Formula: $\mathrm{BiOC}_{19} \mathrm{H}_{17}$

Molecular Weight: 490.36

Elemental Analysis: C: 48.52; Bi: 44.43; H: 3.64; O: 3.40

\begin{tabular}{|c|c|c|c|c|c|}
\hline \multirow[b]{2}{*}{ Date of report } & \multirow{2}{*}{\multicolumn{2}{|c|}{$5 / 10 / 2019 \quad 2: 34: 38 \mathrm{PM}$}} & & & \\
\hline & & & & & \\
\hline User ID & Administrat & & & & \\
\hline Comments & TLG_1_146 & [Hyvl] & & & \\
\hline DATE \& TME & $5 / 10 / 20$ & 19 1:54:48 PM & & P_ID & EA LAB \\
\hline SAMPLE ID & 19295 & & & USER ID & Administrator \\
\hline WEIGHT (mg) & 2.538 & & & MODE & $\mathrm{CHN}$ \\
\hline & & CARBON & $48.645 \%$ & & \\
\hline & & HYDROGEN & $3.680 \%$ & & \\
\hline & & NITROGEN & $-.012 \%$ & & \\
\hline
\end{tabular}

\section{Special Handling}

The sample was combusted in a tin capsule that was crimp-sealed with a die apparatus.

\section{Acknowledgment}

Analytical data were obtained from the CENTC Elemental Analysis Facility at the University of Rochester, funded by NSF CHE-0650456.

Figure S12. EA report for $\mathbf{1 b}$ 


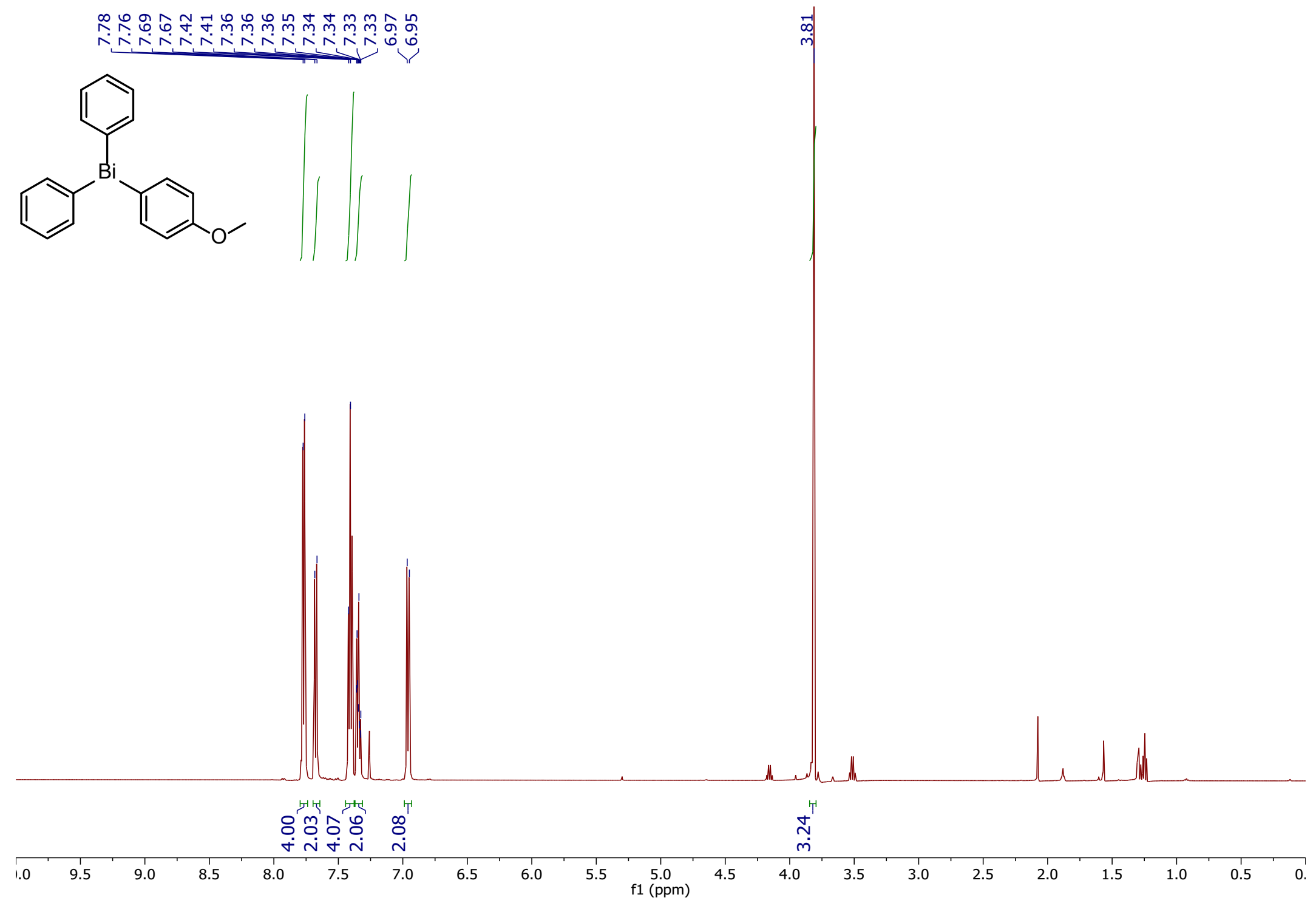

Figure S13. ${ }^{1} \mathrm{H}$ NMR of $\mathbf{1 b}$ in $\mathrm{CDCl}_{3}$ 


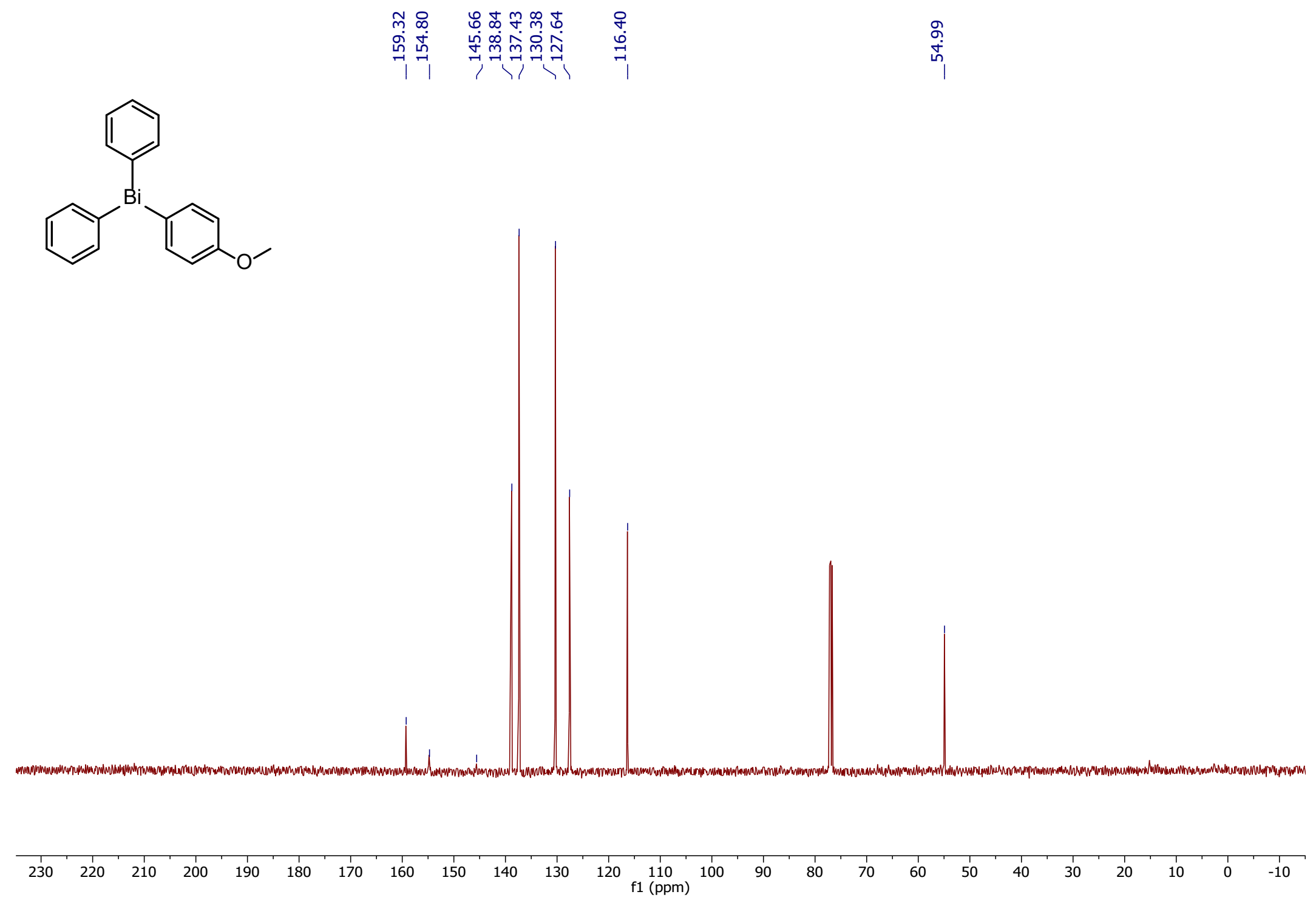

Figure S14. ${ }^{13} \mathrm{C}\left\{{ }^{1} \mathrm{H}\right\}$ NMR of $\mathbf{1 b}$ in $\mathrm{CDCl}_{3}$ 


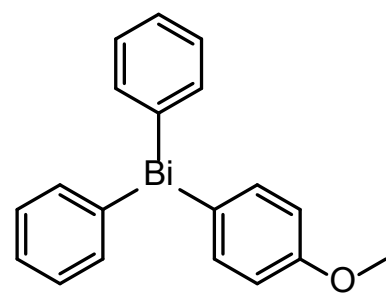

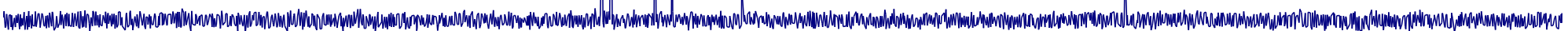

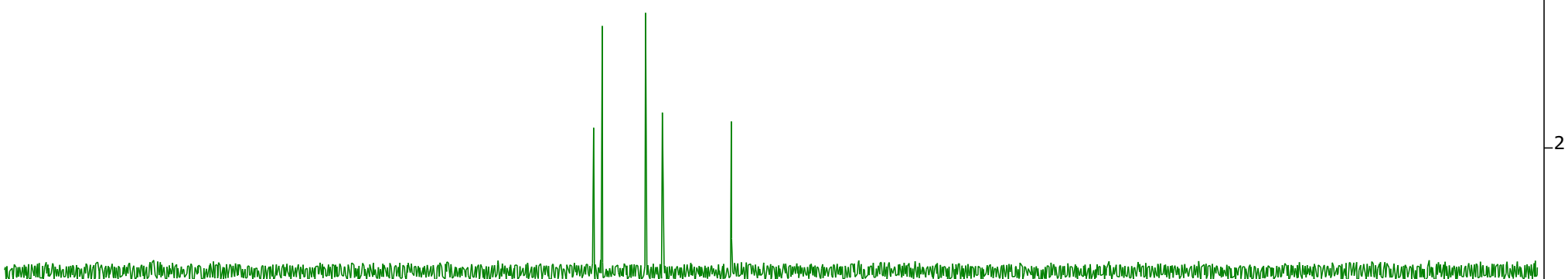

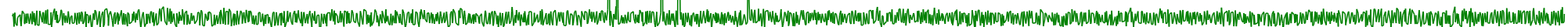

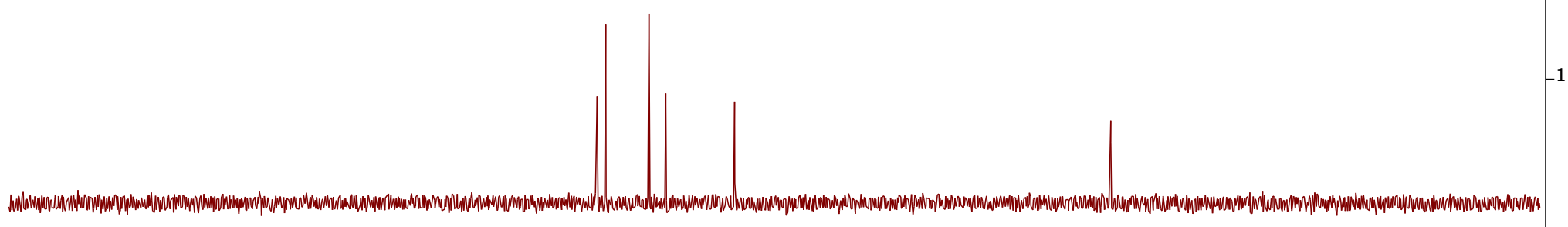

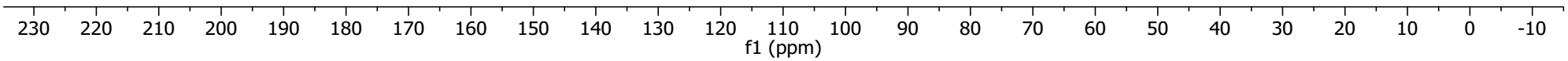

Figure S15. ${ }^{13} \mathrm{C}$ DEPT of $\mathbf{1 b}$ in $\mathrm{CDCl}_{3}$ 


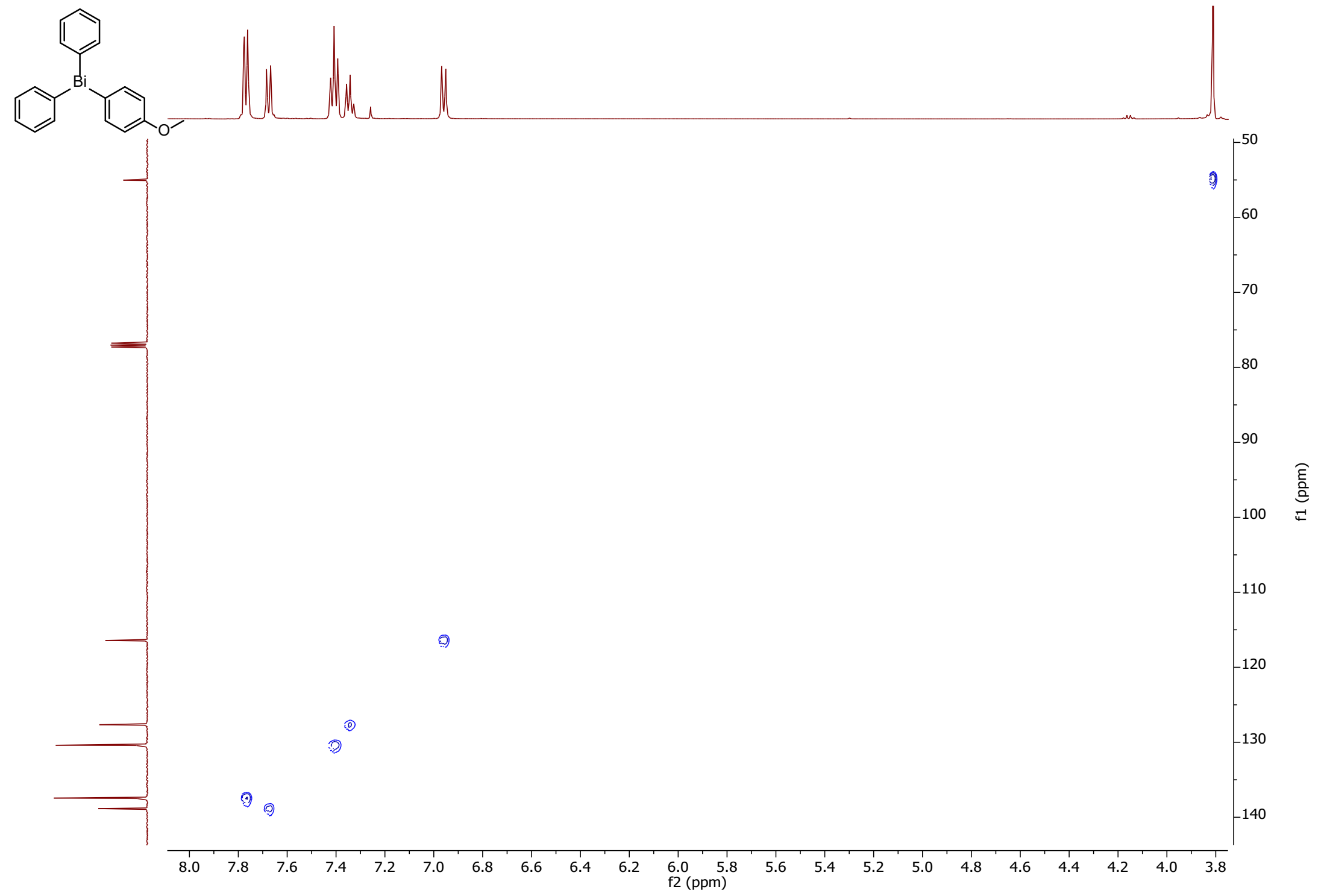

Figure S16. 2D HSQC of $\mathbf{1 b}$ in $\mathrm{CDCl}_{3}$ 


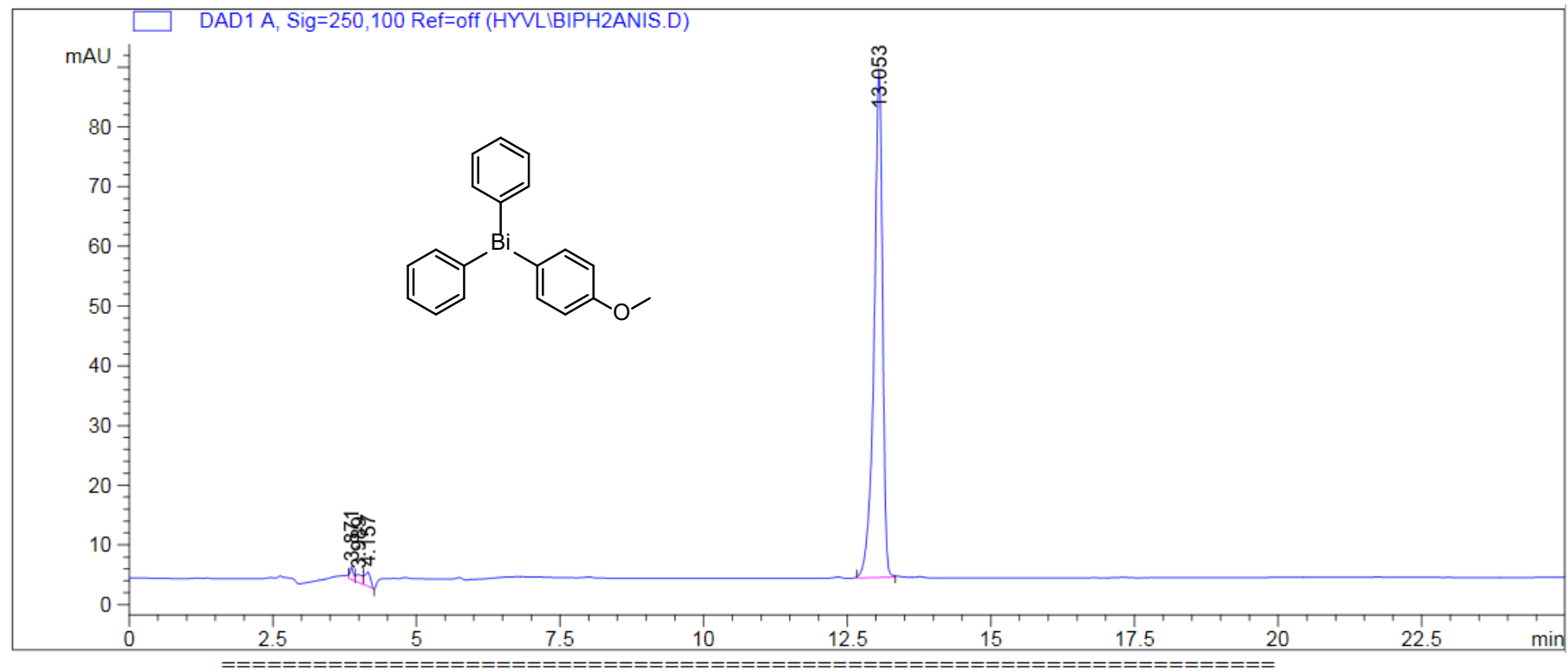

Area Percent Report

$\begin{array}{lcccc}\text { Sorted By } & : & \text { Signal } & & \\ \text { Multiplier: } & : & 1.0000 & \\ \text { Dilution: } & : & 1.0000 & \\ \text { Sample Amount: } & : & 20.00000 & \text { [ng/ul] } & \text { (not used in calc.) } \\ \text { Use Multiplier \& Dilution Factor with } & \text { ISTDs } & & \end{array}$

Signal 1: DAD1 A, Sig=250,100 Ref=off

\begin{tabular}{|c|c|c|c|c|c|c|}
\hline $\begin{array}{c}\text { Peak } \\
\#\end{array}$ & $\begin{array}{l}\text { RetTime } \\
\text { [min] }\end{array}$ & Type & $\begin{array}{c}\text { Width } \\
\text { [min] }\end{array}$ & $\begin{array}{c}\text { Area } \\
{\left[\mathrm{mAU}^{\star} \mathrm{S}\right]}\end{array}$ & $\begin{array}{l}\text { Height } \\
\text { [mAU] }\end{array}$ & $\begin{array}{c}\text { Area } \\
\frac{\circ}{\partial}\end{array}$ \\
\hline 1 & 3.871 & $\mathrm{BV}$ & 0.0615 & 8.75000 & 2.06416 & 0.9597 \\
\hline 2 & 3.989 & VV & 0.1130 & 11.17959 & 1.31753 & 1.2261 \\
\hline 3 & 4.157 & VV & 0.1015 & 15.79875 & 2.37831 & 1.7327 \\
\hline 4 & 13.053 & $\mathrm{BB}$ & 0.1543 & 876.05383 & 84.94643 & 96.0815 \\
\hline
\end{tabular}

Figure S17. HPLC Chromatogram of $\mathbf{1 b}$ 


\section{NMR Spectra and EA Report of Compound 1c}<smiles>Cc1cc(C)c([Bi](c2ccccc2)c2ccccc2)c(C)c1</smiles>

Chemical Formula: $\mathrm{BiC}_{21} \mathrm{H}_{21}$

Molecular Weight: 482.38

Elemental Analysis: C: 52.29; Bi: 43.32; H: 4.39

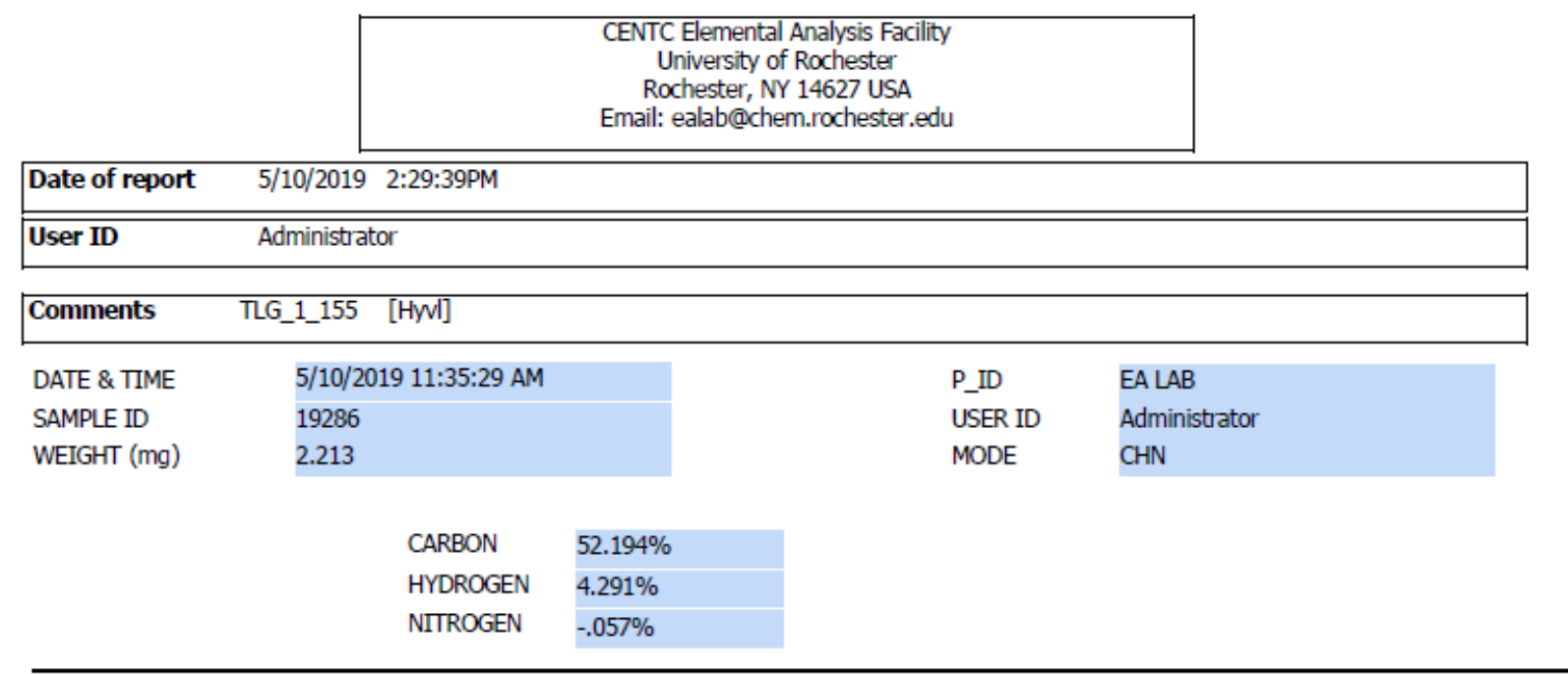

\section{Acknowledgment}

Analytical data were obtained from the CENTC Elemental Analysis Facility at the University of Rochester, funded by NSF CHE-0650456.

\section{Instrumentation}

Microanalysis samples were weighed with a PerkinElmer Model AD6000 Autobalance and their compositions were determined with a PerkinElmer 2400 Series II Analyzer.

Figure S18. EA report for 1c 


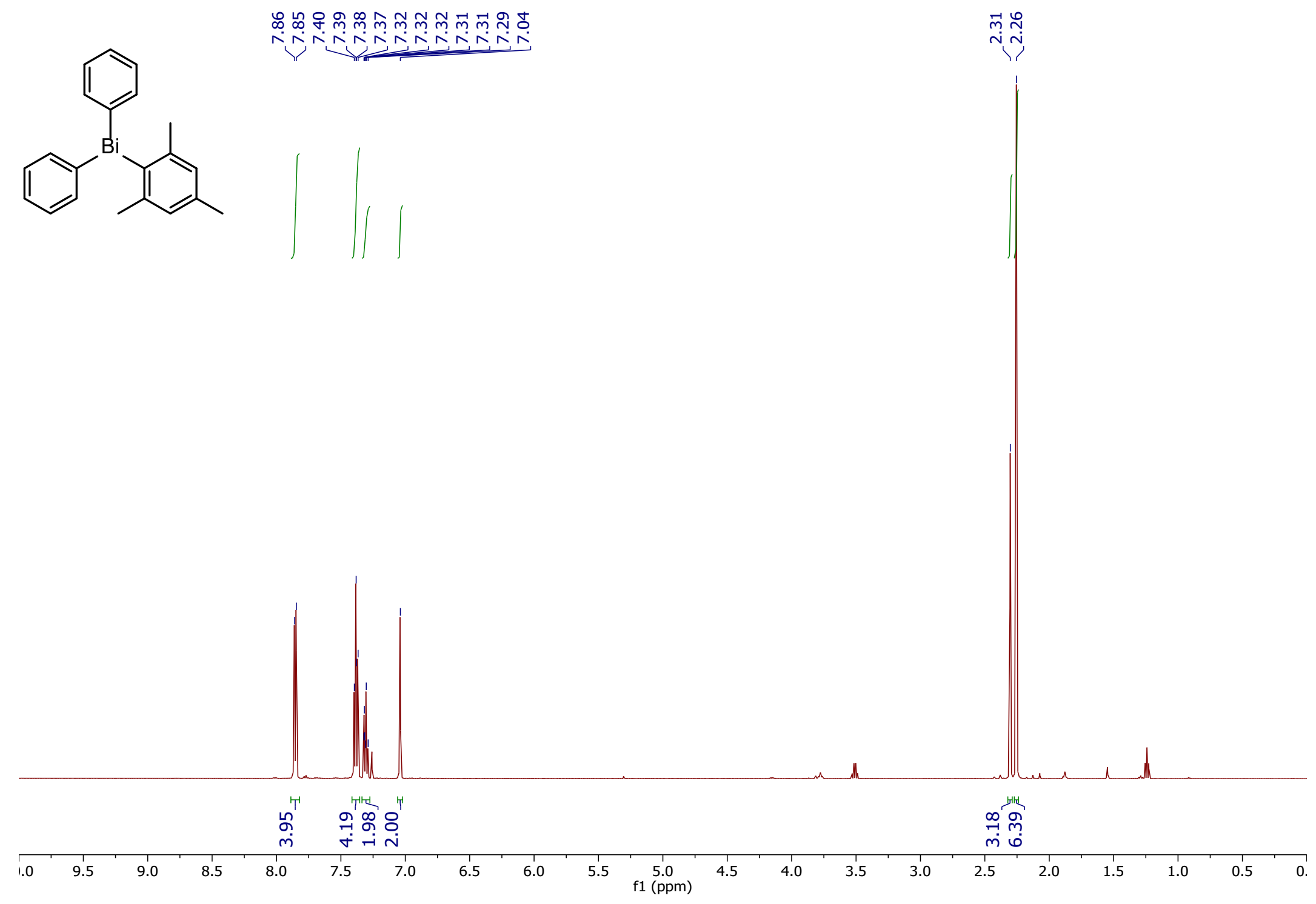

Figure S19. ${ }^{1} \mathrm{H} \mathrm{NMR}$ of $1 \mathrm{c} \mathrm{CDCl}_{3}$ 


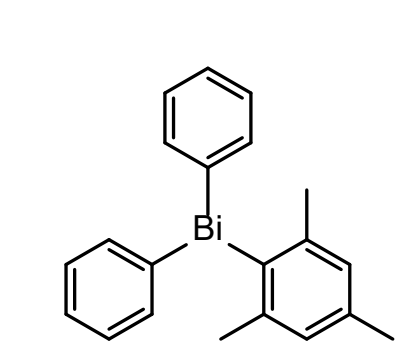

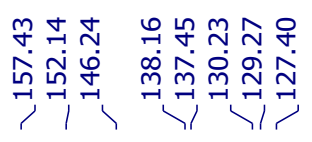

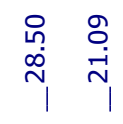
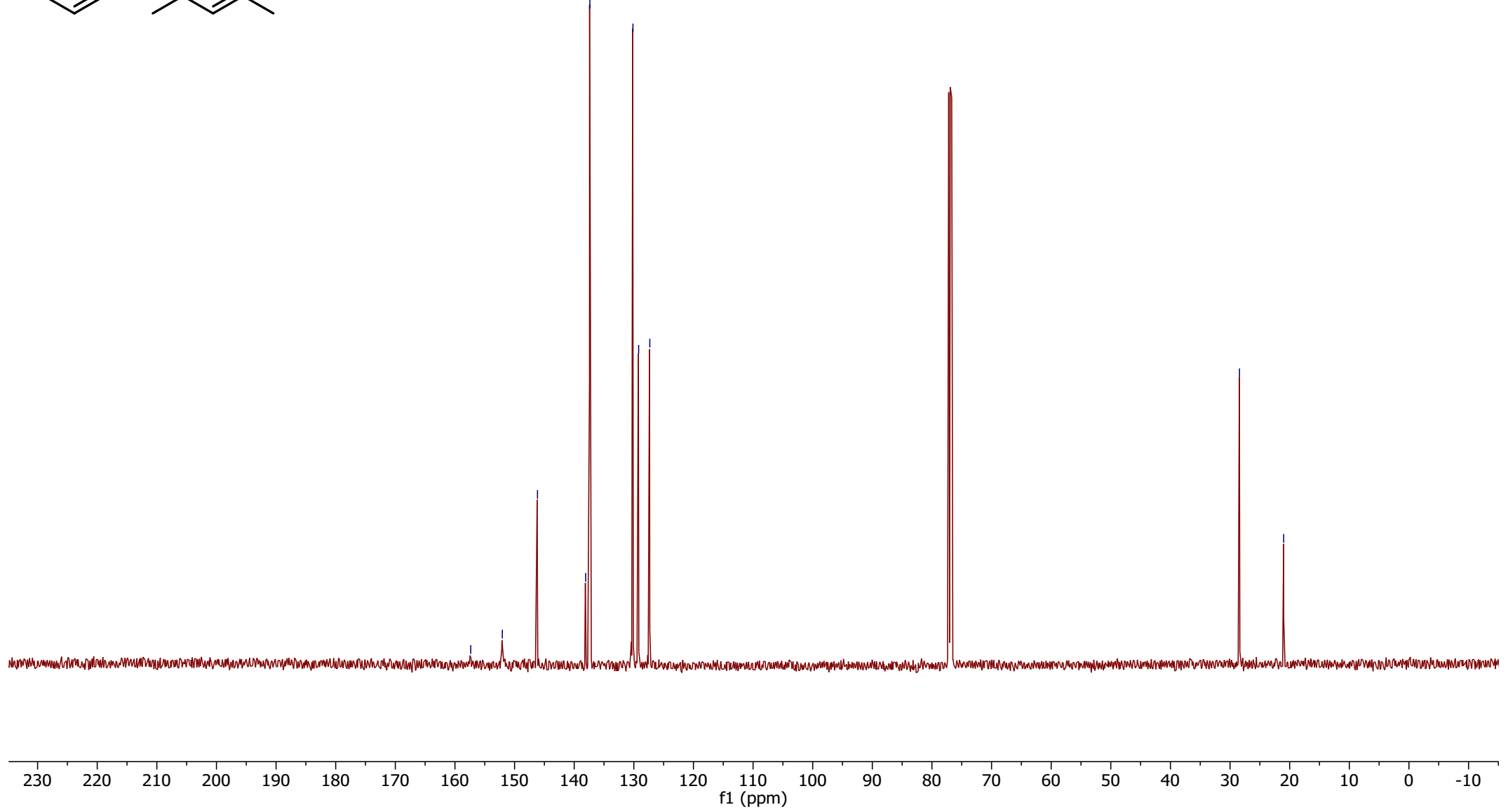

Figure S20. ${ }^{13} \mathrm{C}\left\{{ }^{1} \mathrm{H}\right\}$ NMR of $1 \mathrm{c}$ in $\mathrm{CDCl}_{3}$

S32 


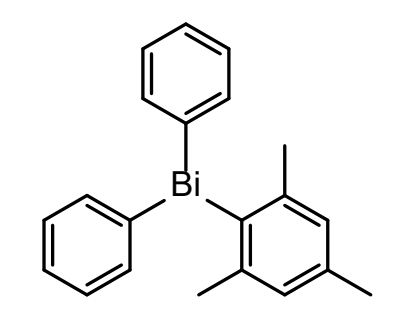

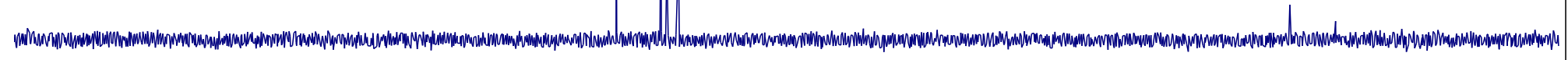

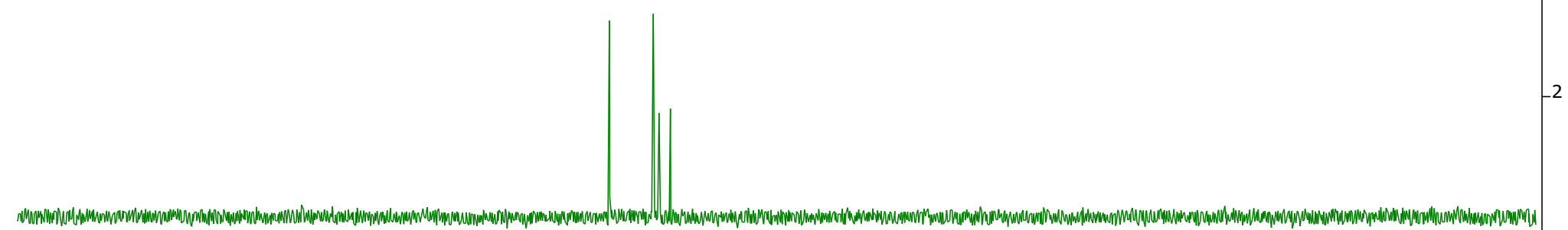

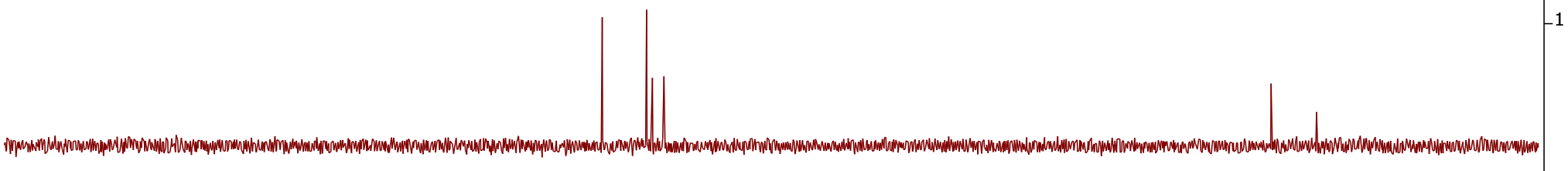

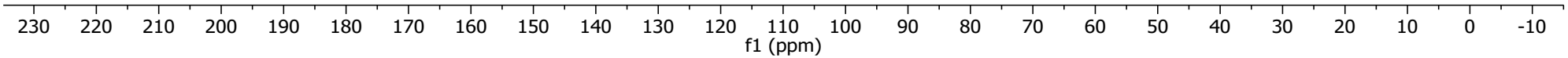

Figure S21. ${ }^{13} \mathrm{C}$ DEPT of $1 \mathrm{c}$ in $\mathrm{CDCl}_{3}$ 


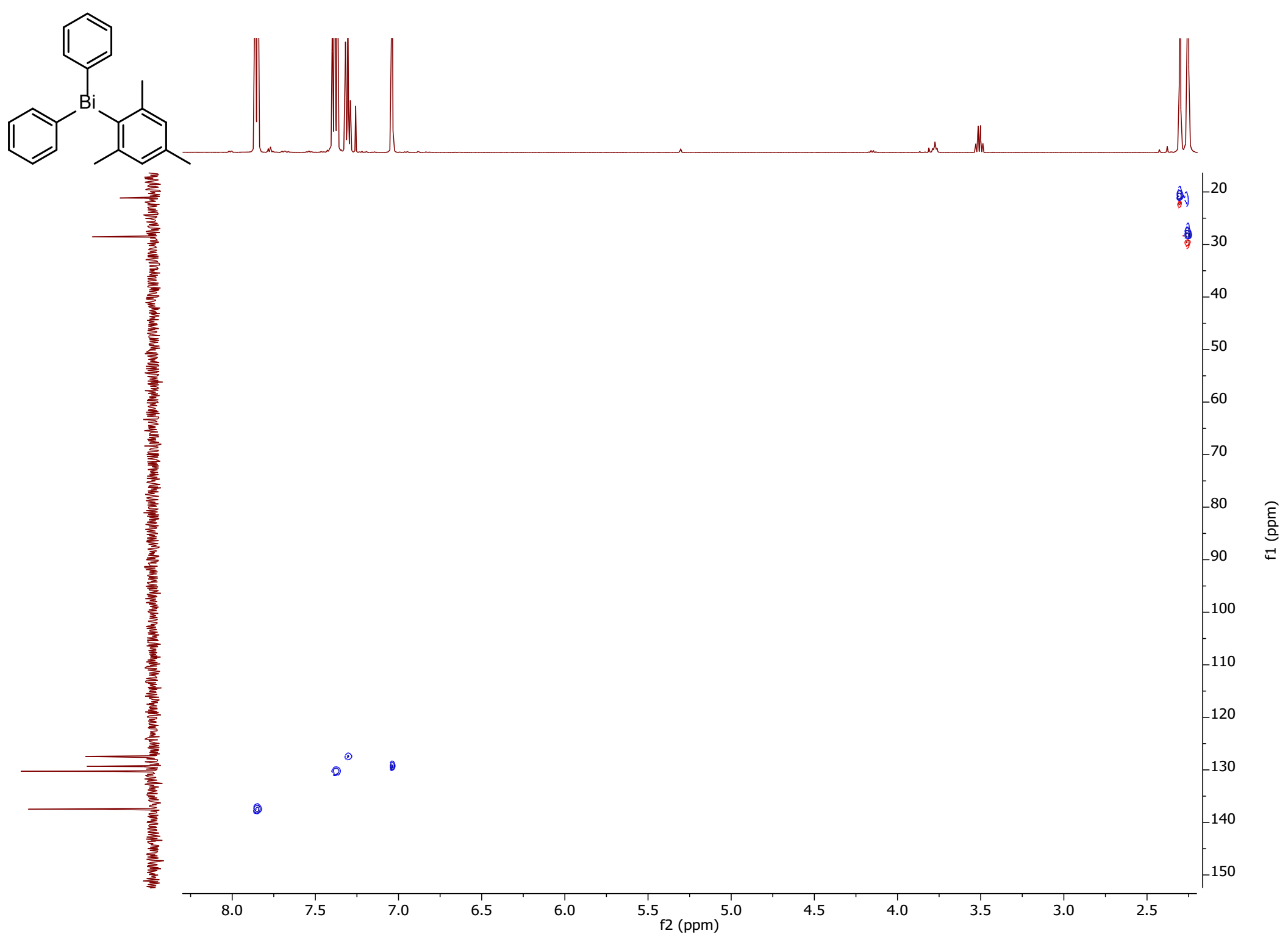

Figure S22. 2D HSQC of $1 \mathrm{c}$ in $\mathrm{CDCl}_{3}$ 


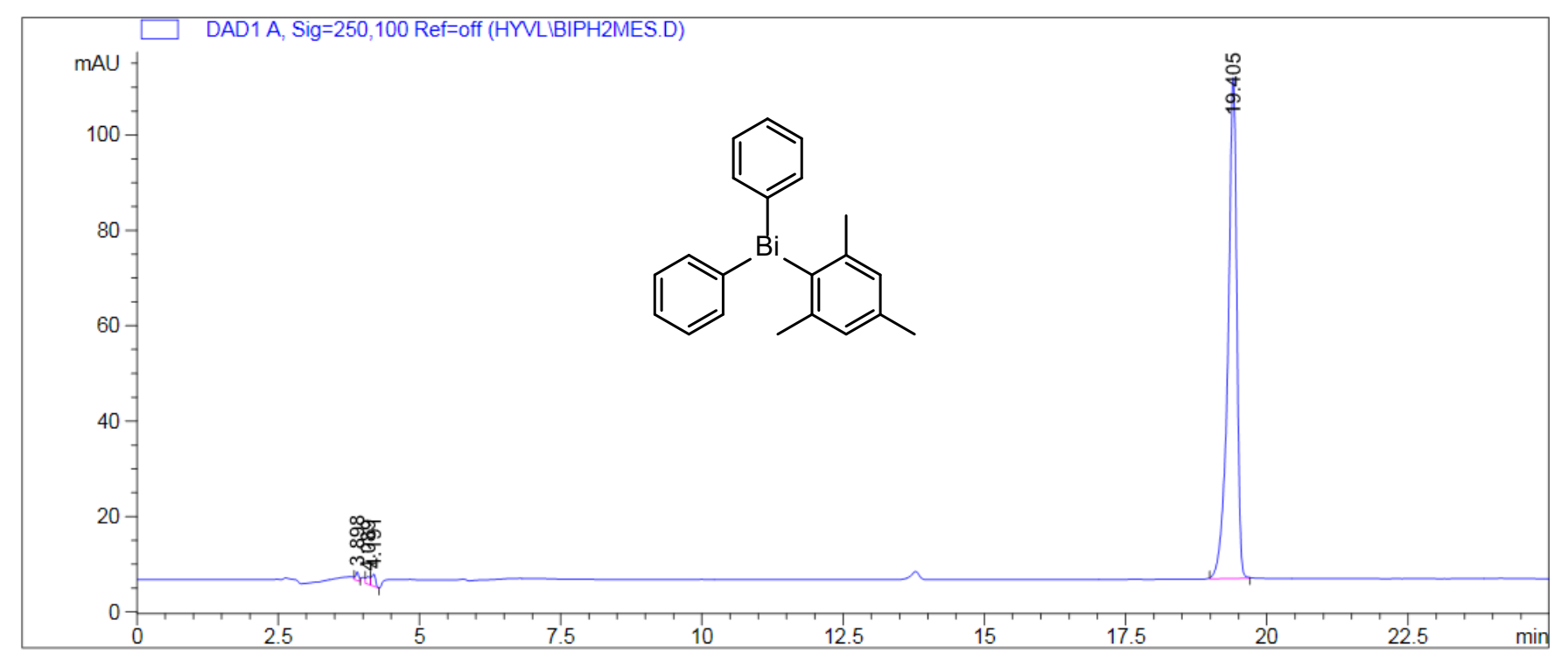

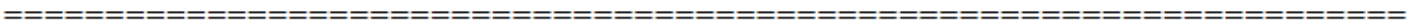

Area Percent Report

Sorted By

Multiplier:

Dilution:

Signal

Sample Amount:

: 1.0000

$: \quad 1.0000$

20.00000

Use Multiplier \& Dilution Factor with ISTDs

[ng/ul] (not used in calc.)

Signal 1: DAD1 A, Sig=250,100 Ref=off

\begin{tabular}{|c|c|c|c|c|c|c|}
\hline $\begin{array}{c}\text { Peak } \\
\#\end{array}$ & $\begin{array}{c}\text { RetTime } \\
\text { [min] }\end{array}$ & Type & $\begin{array}{c}\text { Width } \\
\text { [min] }\end{array}$ & $\begin{array}{c}\text { Area } \\
{\left[\mathrm{mAU}^{*} \mathrm{~S}\right]}\end{array}$ & $\begin{array}{l}\text { Height } \\
\text { [mAU] }\end{array}$ & $\begin{array}{c}\text { Area } \\
\quad \&\end{array}$ \\
\hline & & & & ------- & ---- & 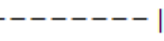 \\
\hline 1 & 3.898 & $\mathrm{BV}$ & 0.0569 & 6.90854 & 1.79331 & 0.5888 \\
\hline 2 & 4.089 & VV & 0.0740 & 8.17104 & 1.58943 & 0.6964 \\
\hline 3 & 4.191 & VV & 0.0726 & 12.11588 & 2.49681 & 1.0325 \\
\hline 4 & 19.405 & $\mathrm{BB}$ & 0.1612 & 1146.20776 & 105.09236 & 97.6823 \\
\hline
\end{tabular}

Figure S23. HPLC Chromatogram of 1c 


\section{NMR Spectra and EA Report of Compound 1d}<smiles>COC(=O)c1ccc([Bi](c2ccccc2)c2ccccc2)cc1</smiles>

Chemical Formula: $\mathrm{BiO}_{2} \mathrm{C}_{20} \mathrm{H}_{17}$

Molecular Weight: 498.33

Elemental Analysis: C: 48.20; Bi: 41.94; H: 3.44; O: 6.42

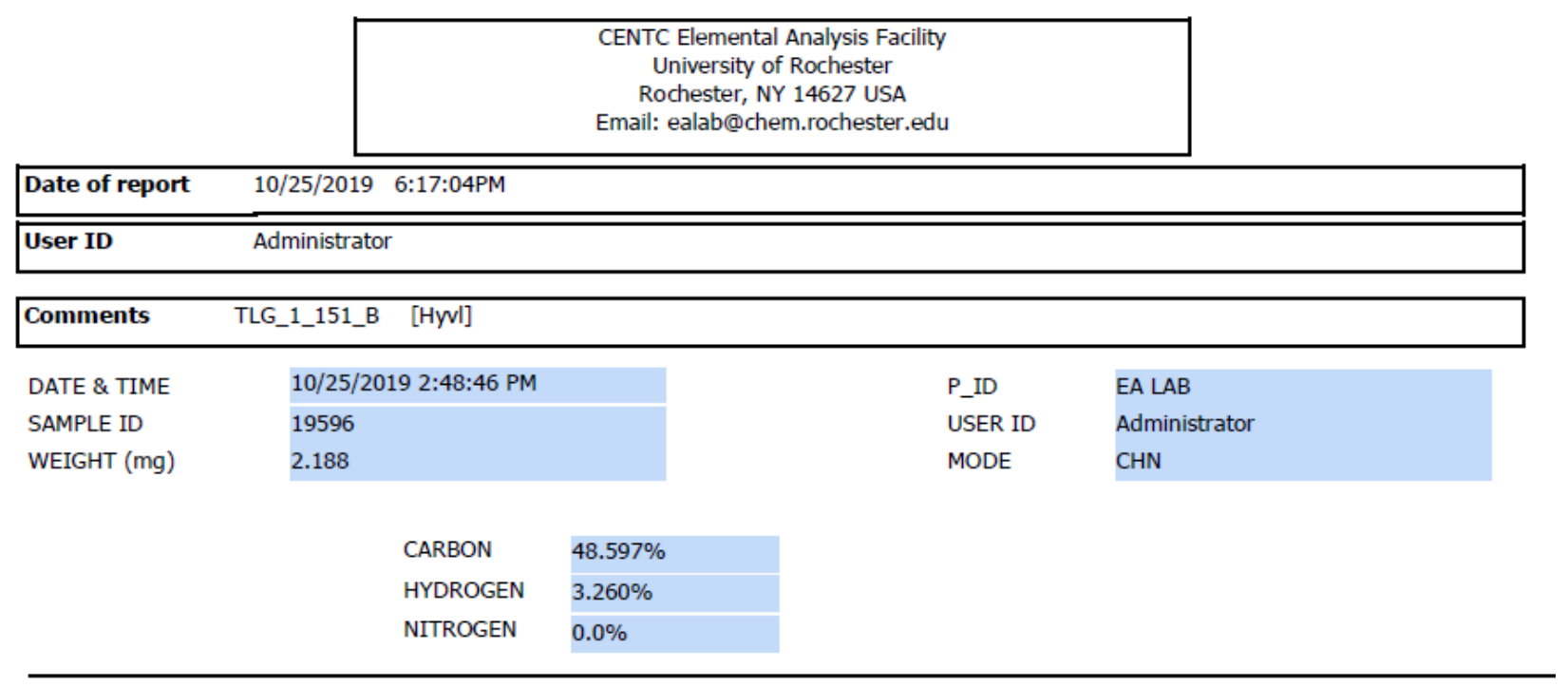

Acknowledgment

Analytical data were obtained from the CENTC Elemental Analysis Facility at the University of Rochester, funded by NSF CHE-0650456.

Figure S24. EA report for $1 d$ 


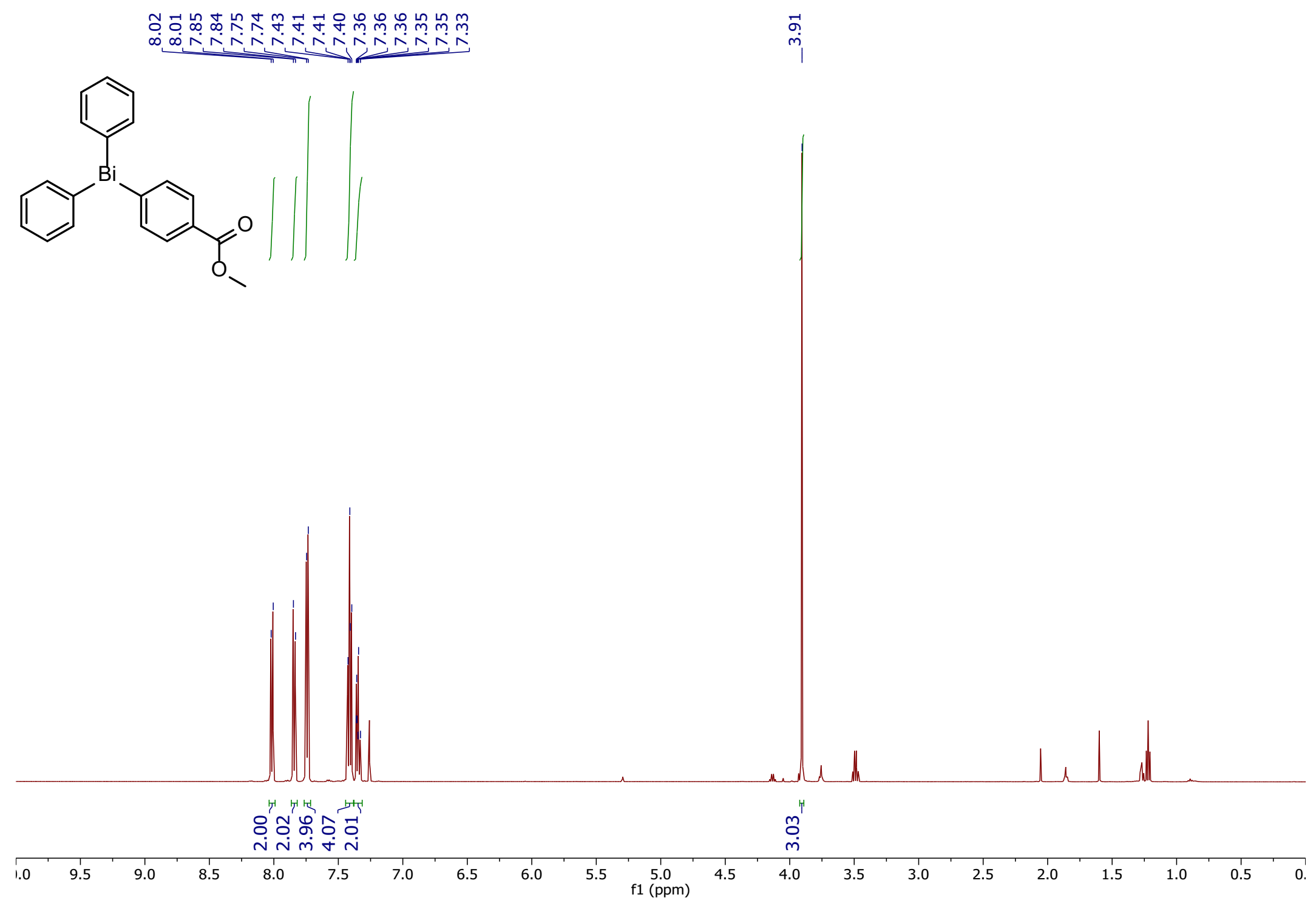

Figure S25. ${ }^{1} \mathrm{H}$ NMR of $\mathbf{1 d}$ in $\mathrm{CDCl}_{3}$ 


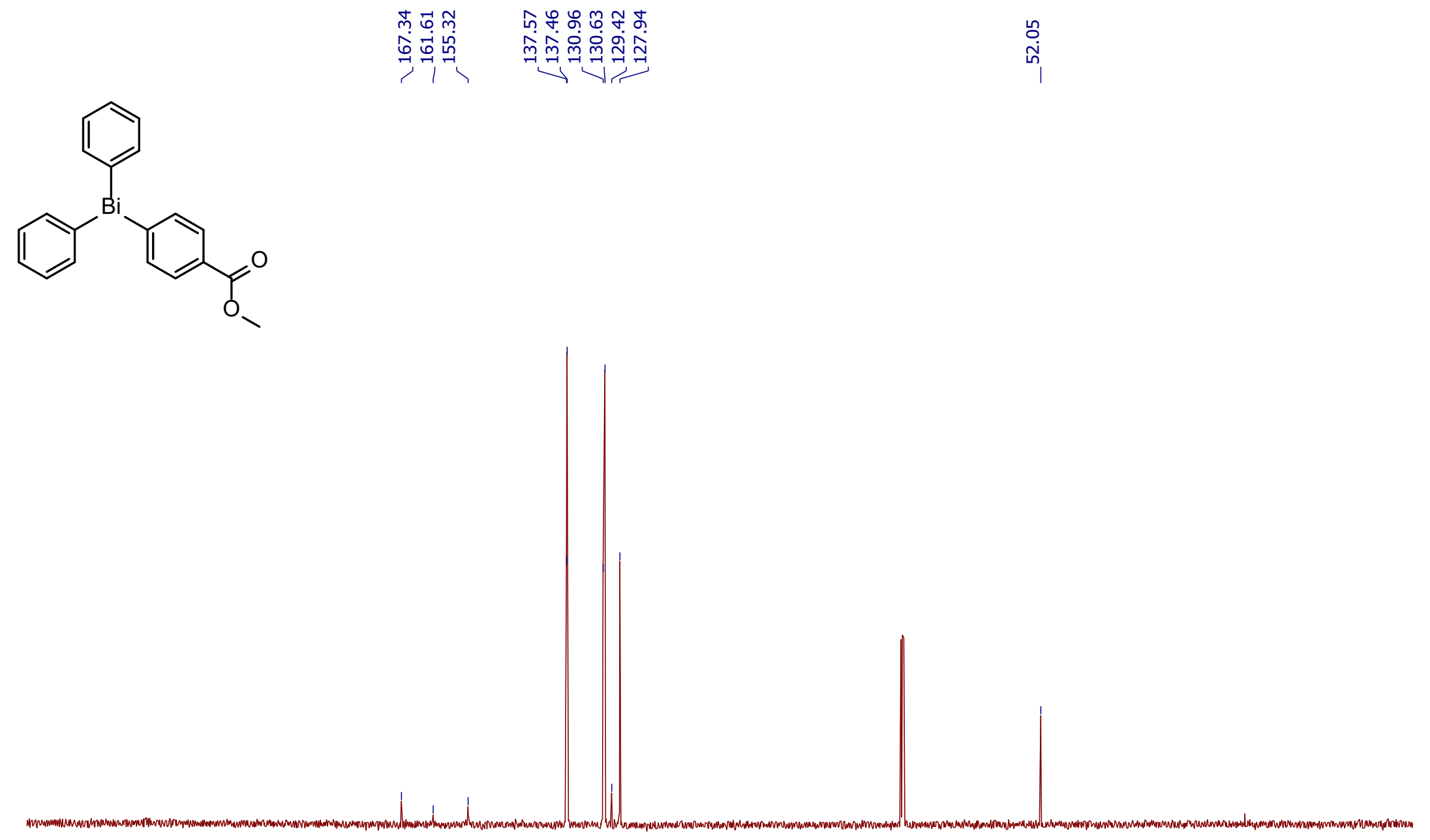

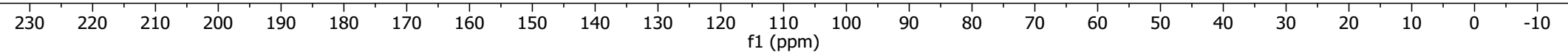

Figure S26. ${ }^{13} \mathrm{C}\left\{{ }^{1} \mathrm{H}\right\}$ NMR of $\mathbf{1 d}$ in $\mathrm{CDCl}_{3}$ 


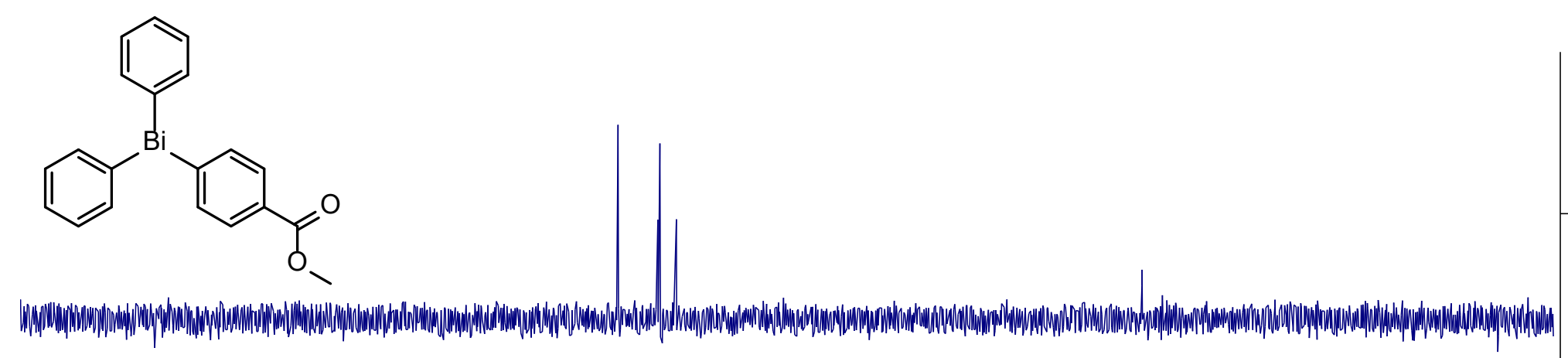

I.

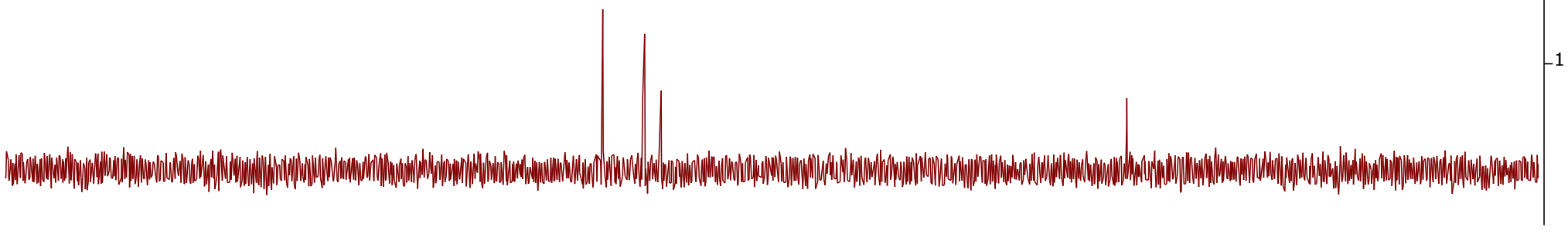

\begin{tabular}{lllllllllllllllllllllllll}
\hline 230 & 220 & 210 & 200 & 190 & 180 & 170 & 160 & 150 & 140 & 130 & 120 & 110 & 100 & 90 & 80 & 70 & 60 & 50 & 40 & 30 & 20 & 10 & 0 & -10
\end{tabular}

Figure S27. ${ }^{13} \mathrm{C}$ DEPT of $\mathbf{1 d}$ in $\mathrm{CDCl}_{3}$ 


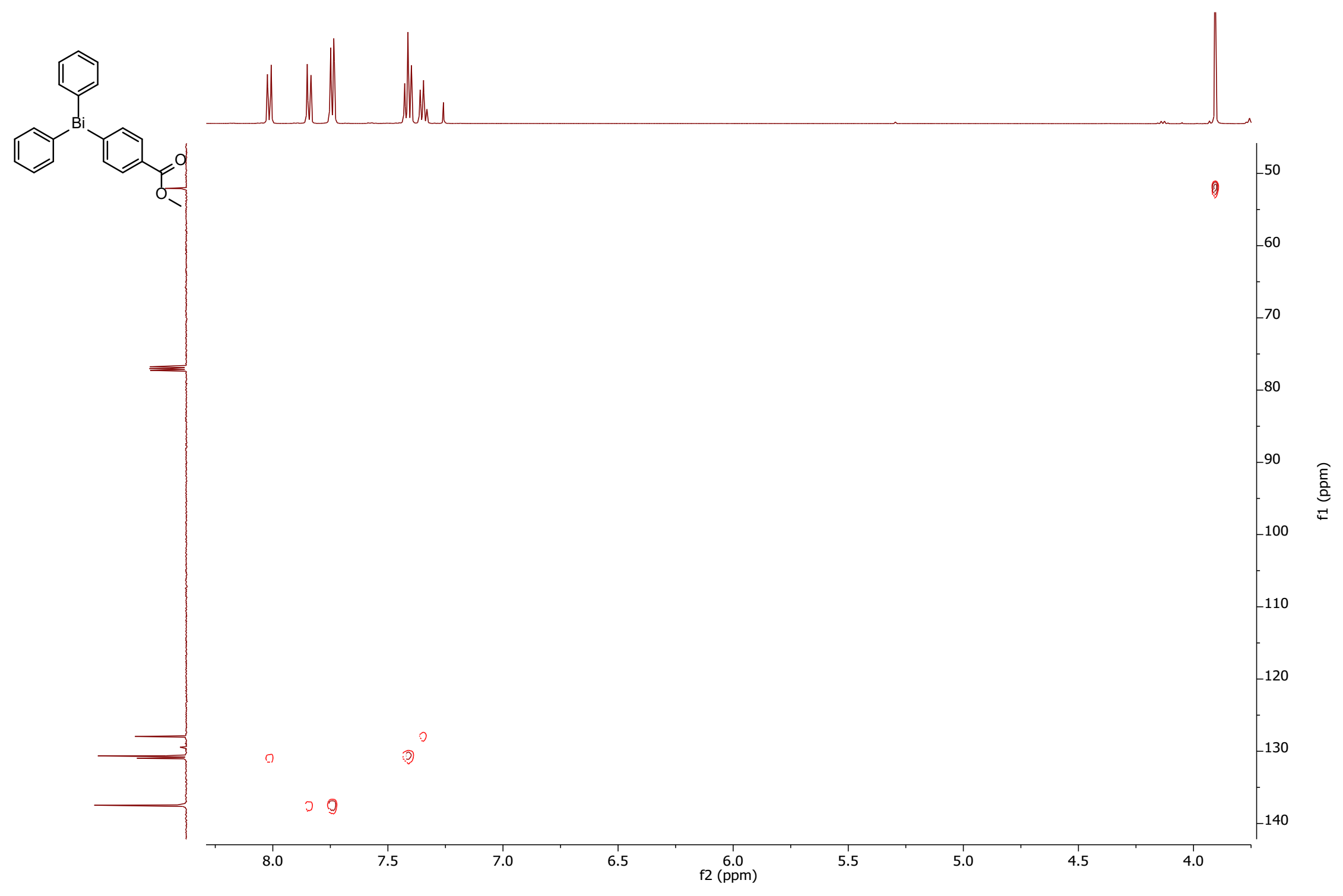

Figure S28. 2D HSQC of $\mathbf{1 d}$ in $\mathrm{CDCl}_{3}$ 


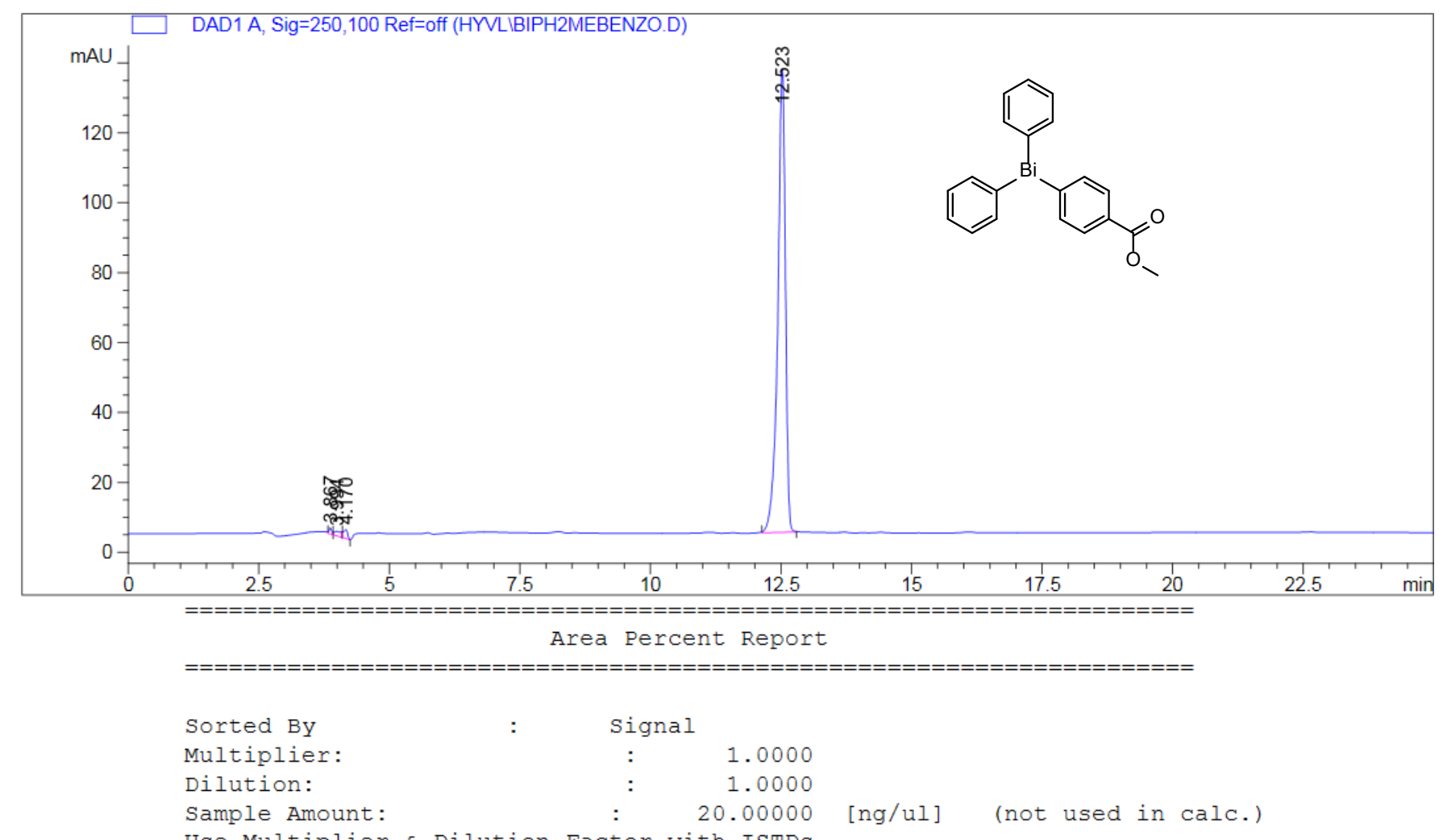

Use Multiplier \& Dilution Factor with ISTDs

Signal 1: DAD1 A, Sig=250,100 Ref=off

\begin{tabular}{|c|c|c|c|c|c|c|}
\hline $\begin{array}{c}\text { Peak } \\
\#\end{array}$ & $\begin{array}{c}\text { RetTime } \\
\text { [min] }\end{array}$ & Type & $\begin{array}{c}\text { Width } \\
\text { [min] }\end{array}$ & $\begin{array}{c}\text { Area } \\
{\left[\mathrm{mAU}^{*} \mathrm{~s}\right]}\end{array}$ & $\begin{array}{l}\text { Height } \\
\text { [mAU] }\end{array}$ & $\begin{array}{c}\text { Area } \\
\&\end{array}$ \\
\hline 1 & 67 & $\mathrm{BV}$ & 74 & 6.39290 & 1.71725 & 0.4643 \\
\hline 2 & 3.994 & VB & 0.1 & 12.02068 & 1.34342 & 0.8730 \\
\hline 3 & 4.170 & $\mathrm{BV}$ & 0.0775 & 13.59000 & 2.57776 & 0.9870 \\
\hline 4 & 12.523 & $\mathrm{BB}$ & 0.1522 & 1344.91504 & 132.69420 & 97.6757 \\
\hline
\end{tabular}

Figure S29. HPLC Chromatogram of 1d 


\section{NMR Spectra and EA Report of Compound 1e}<smiles>N#Cc1ccc([Bi](c2ccccc2)c2ccccc2)cc1</smiles>

Chemical Formula: $\mathrm{BiNC}_{19} \mathrm{H}_{14}$

Molecular Weight: 465.31

Elemental Analysis: C: 49.04; Bi: 44.91; H: 3.03; N: 3.01

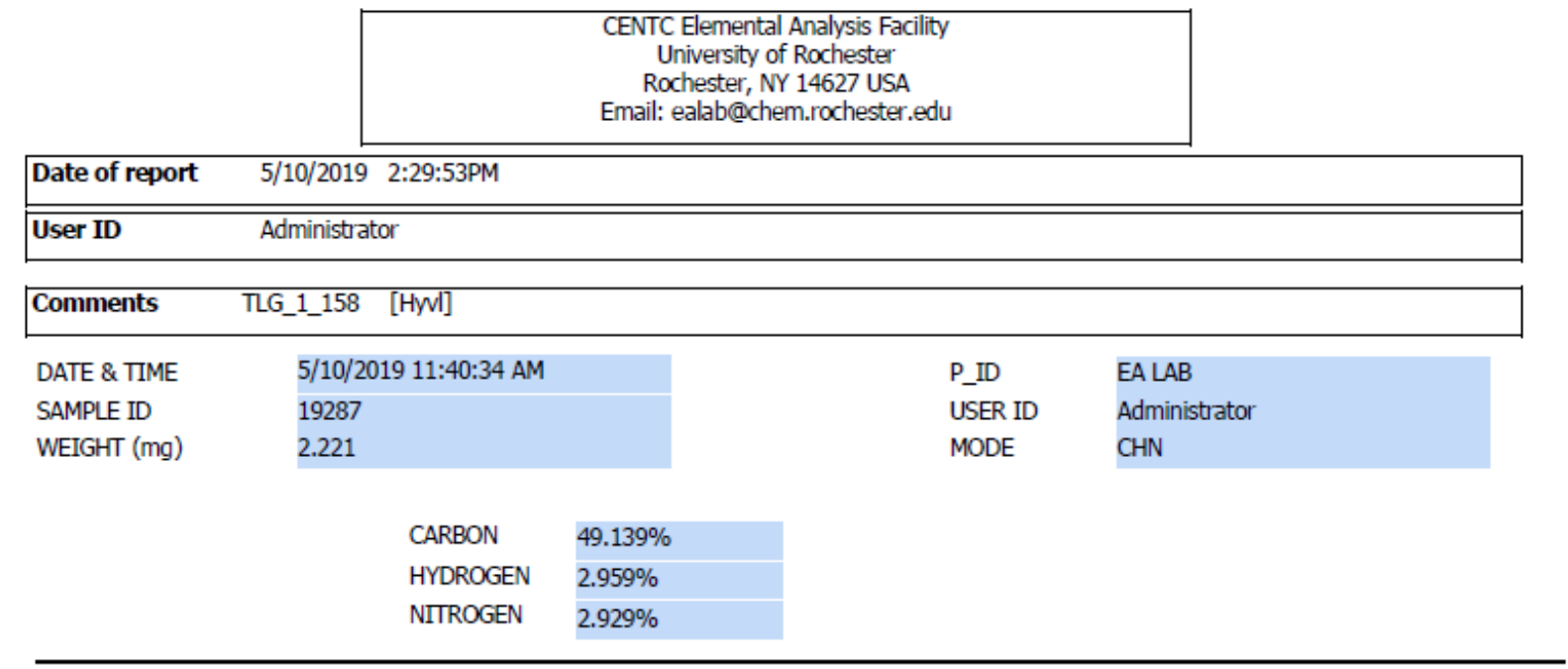

\section{Acknowledgment}

Analytical data were obtained from the CENTC Elemental Analysis Facility at the University of Rochester, funded by NSF CHE-0650456.

\section{Instrumentation}

Microanalysis samples were weighed with a PerkinElmer Model AD6000 Autobalance and their compositions were determined with a PerkinElmer 2400 Series II Analyzer.

Figure S30. EA report for 1e 


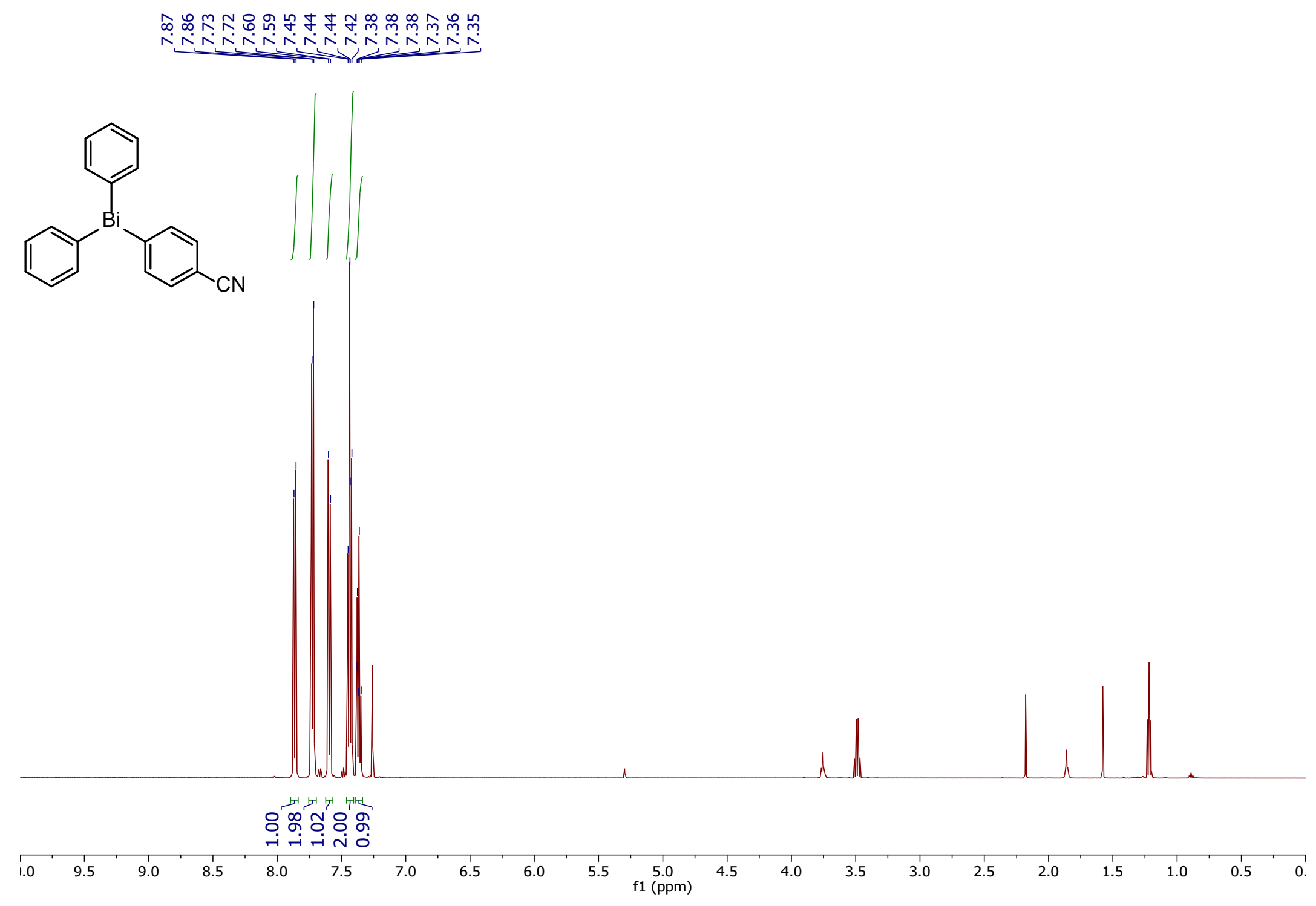

Figure S31. ${ }^{1} \mathrm{H}$ NMR of $1 e$ in $\mathrm{CDCl}_{3}$ 


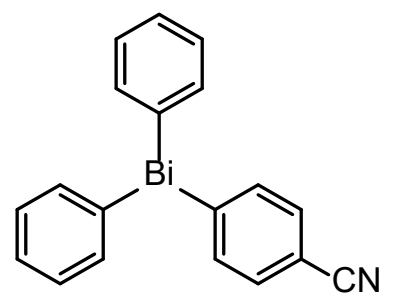

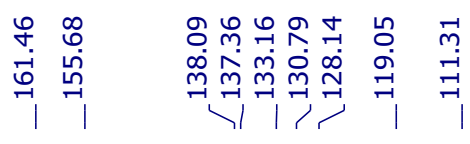

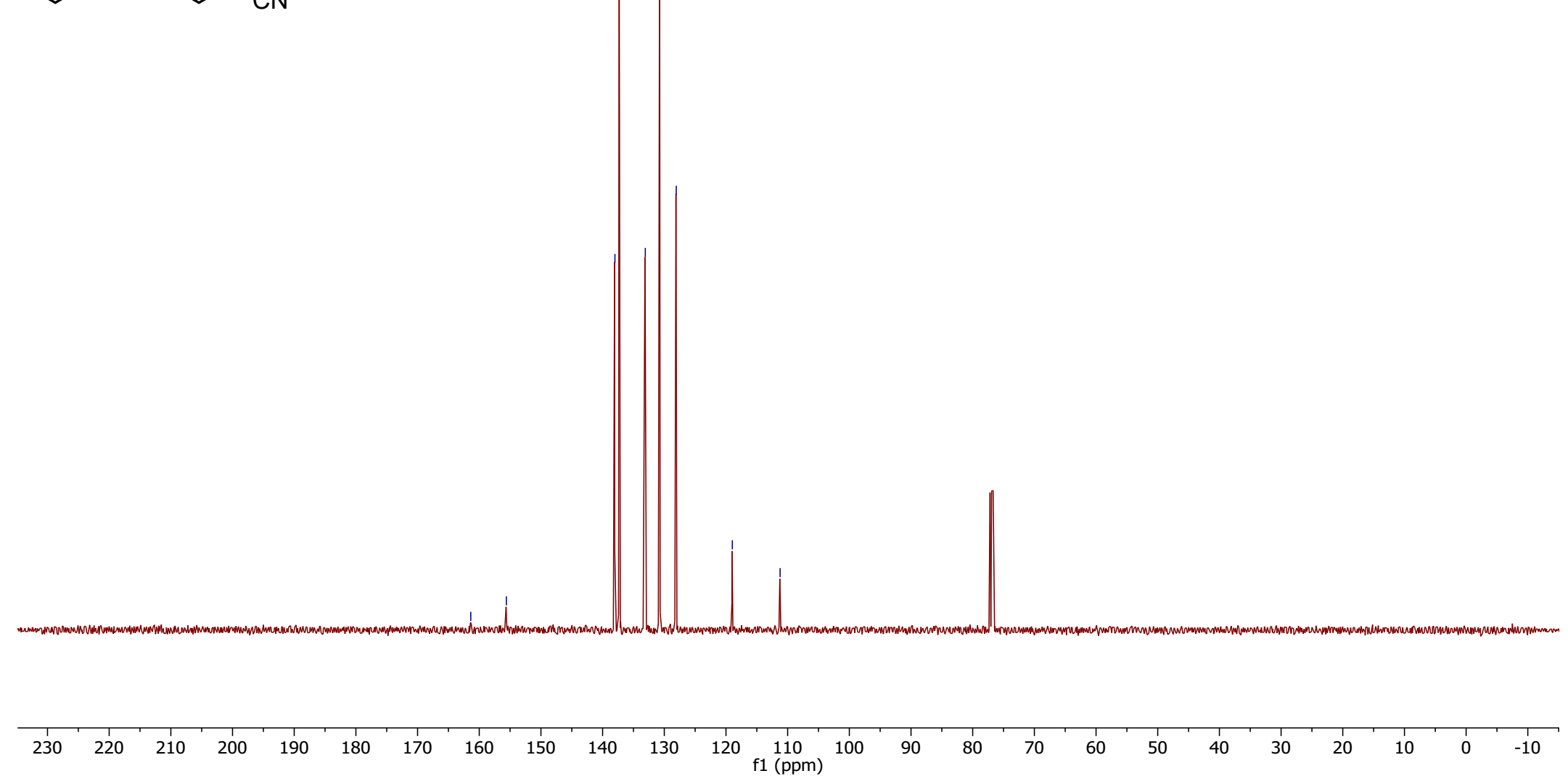

Figure S32. ${ }^{13} \mathrm{C}\left\{{ }^{1} \mathrm{H}\right\}$ NMR of $1 \mathrm{e}$ in $\mathrm{CDCl}_{3}$

S44 

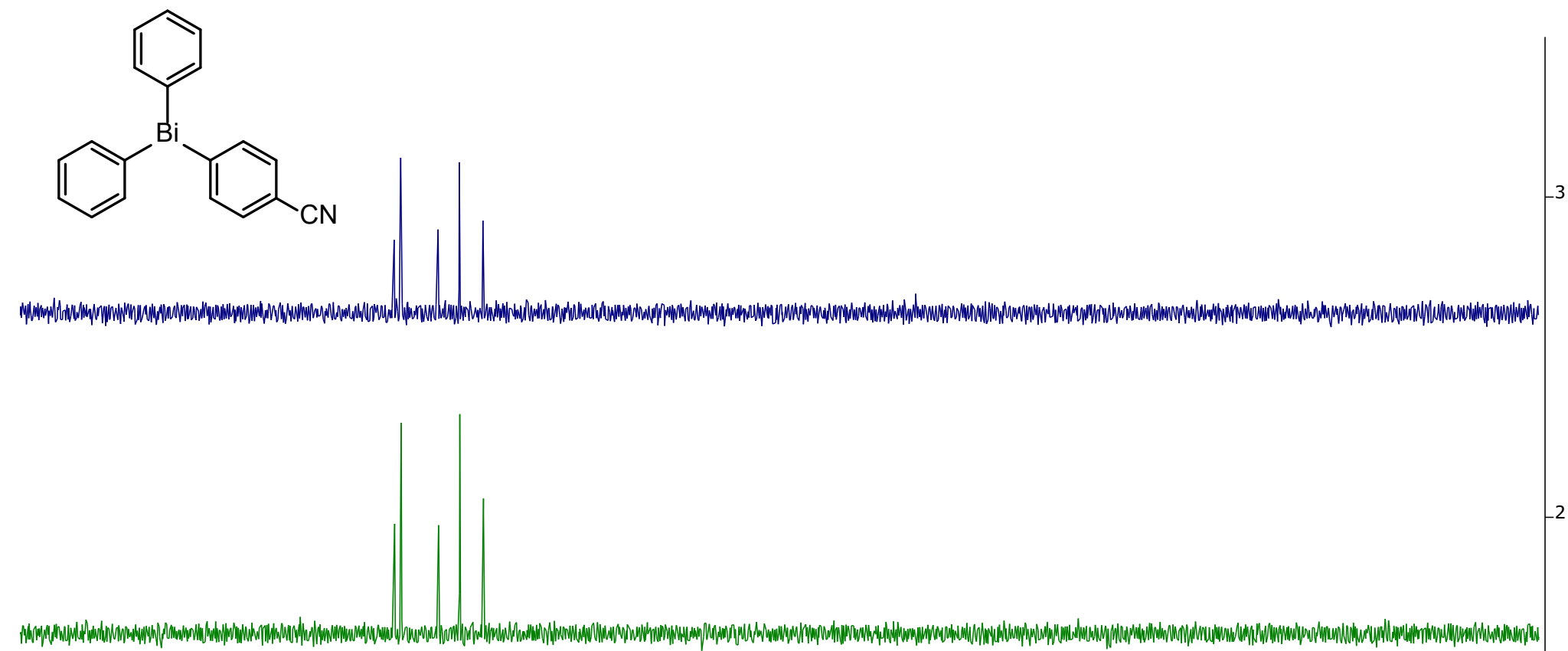

I.

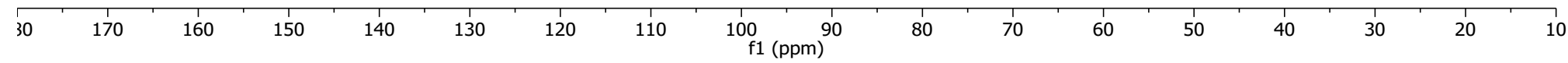

Figure S33. ${ }^{13} \mathrm{C}$ DEPT of $1 \mathrm{e}$ in $\mathrm{CDCl}_{3}$ 


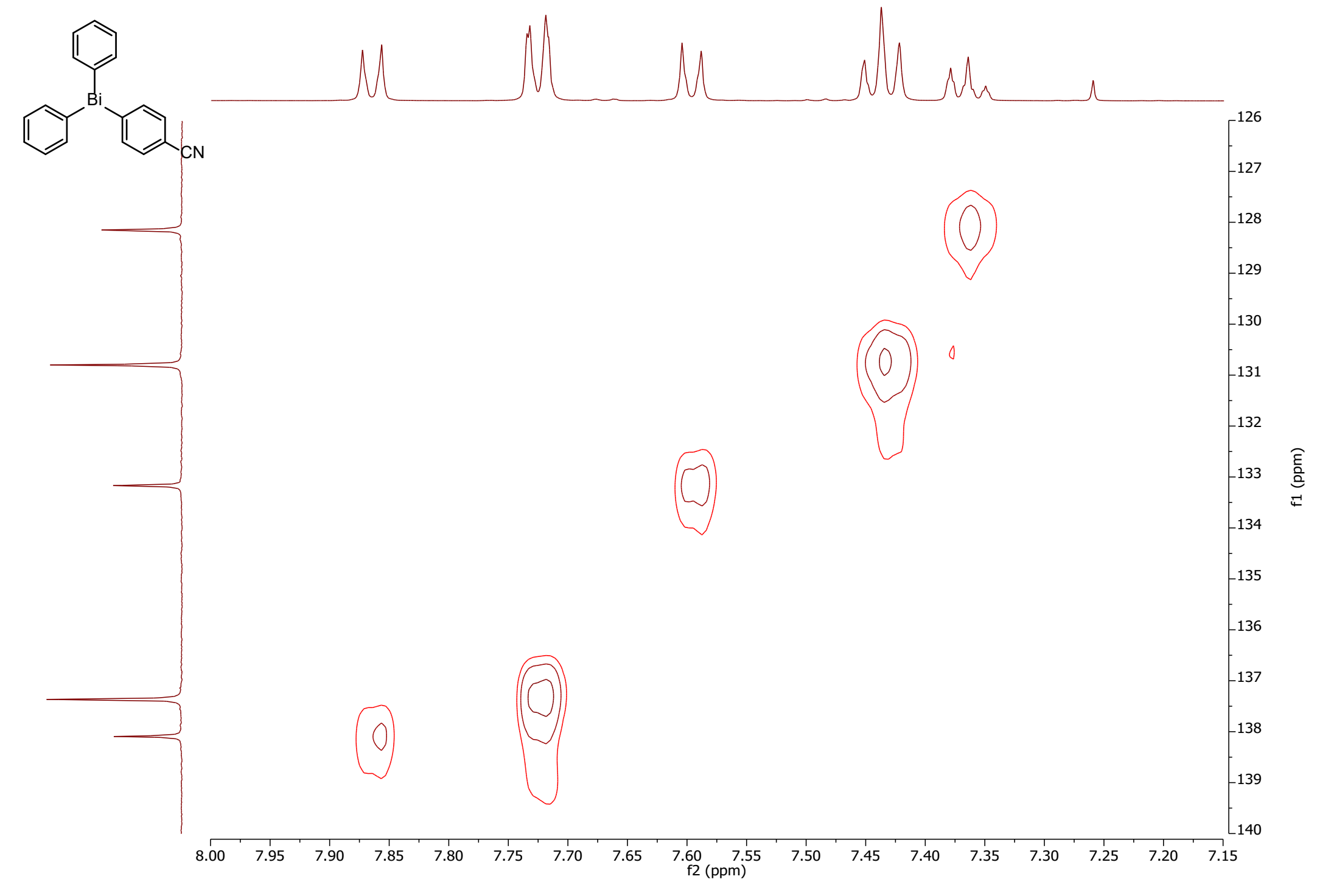

Figure S34. 2D HSQC of $1 e$ in $\mathrm{CDCl}_{3}$ 


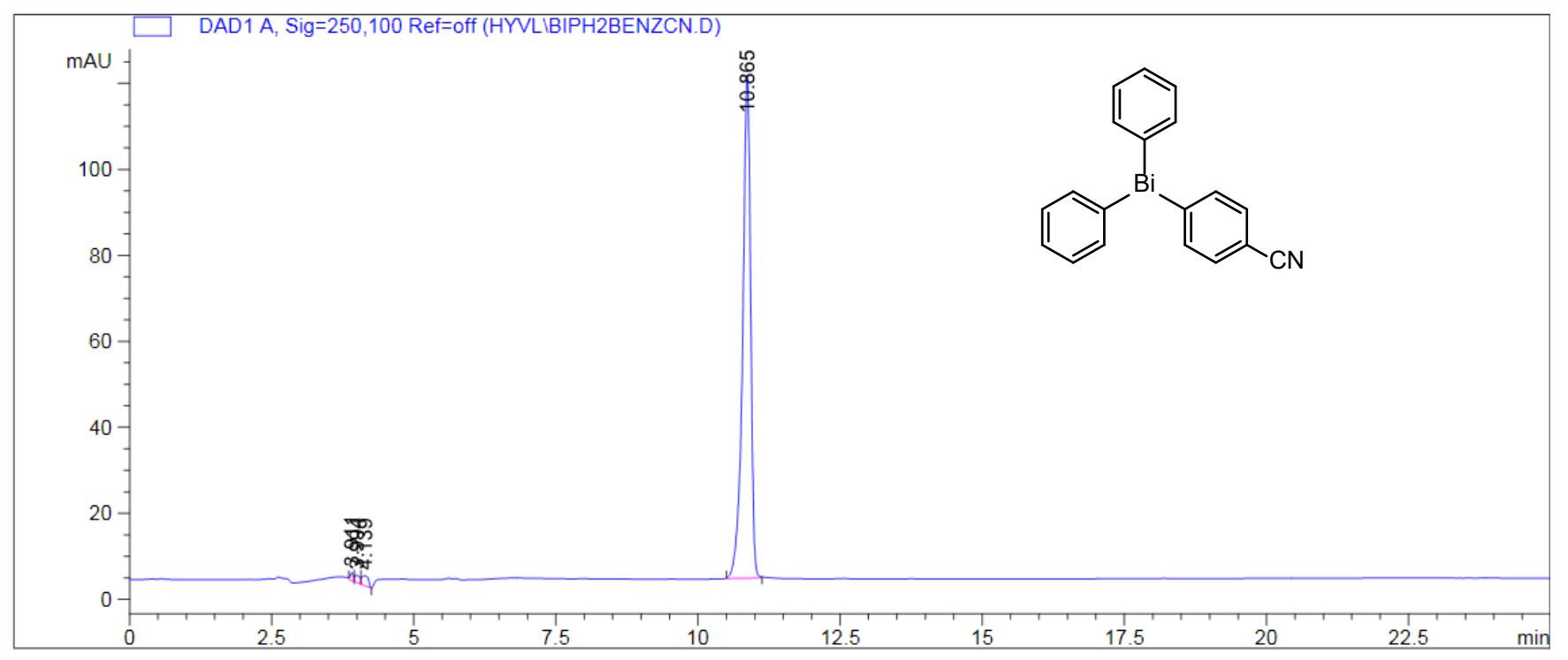

Area Percent Report

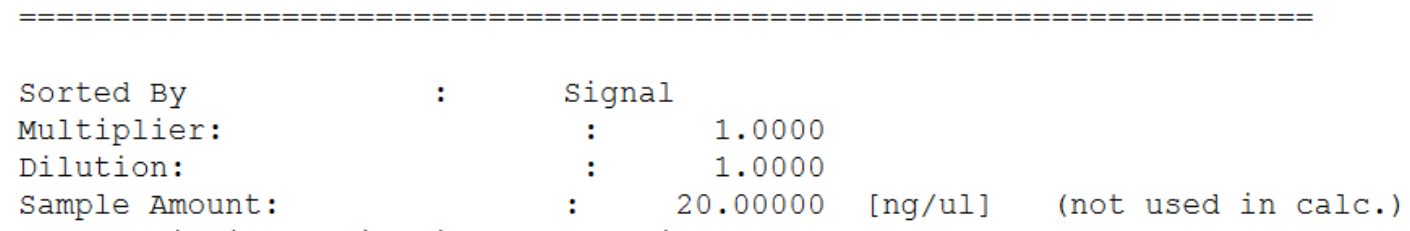

Signal 1: DAD1 A, Sig=250,100 Ref=off

\begin{tabular}{|c|c|c|c|c|c|c|}
\hline $\begin{array}{c}\text { Peak } \\
\#\end{array}$ & $\begin{array}{c}\text { RetTime } \\
\text { [min] }\end{array}$ & Type & $\begin{array}{c}\text { Width } \\
\text { [min] }\end{array}$ & $\begin{array}{c}\text { Area } \\
{\left[\mathrm{mAU}{ }^{\star} \mathrm{s}\right]}\end{array}$ & $\begin{array}{l}\text { Height } \\
\text { [mAU] }\end{array}$ & $\begin{array}{c}\text { Area } \\
\frac{\circ}{0}\end{array}$ \\
\hline 1 & $\begin{array}{ll}3 & 911\end{array}$ & BV & 0.0556 & 6.93817 & 185545 & م. 5882 \\
\hline 2 & 3.994 & VV & 0.0904 & 10.98717 & 1.67865 & 0.9315 \\
\hline 3 & 4.139 & VV & 0.1026 & 17.91453 & 2.30757 & 1.5188 \\
\hline 4 & 10.865 & $\mathrm{BB}$ & 0.1480 & 1143.66382 & 117.06699 & 96.9614 \\
\hline
\end{tabular}

Figure S35. HPLC Chromatogram of 1e 


\section{NMR Spectra and EA Report of Compound $1 f$}<smiles>Cc1cc(C)c([Bi](c2ccccc2)c2c(C)cc(C)cc2C)c(C)c1</smiles>

Chemical Formula: $\mathrm{BiC}_{24} \mathrm{H}_{27}$

Molecular Weight: 524.46

Elemental Analysis: C: 54.96; Bi: 39.85; H: 5.19

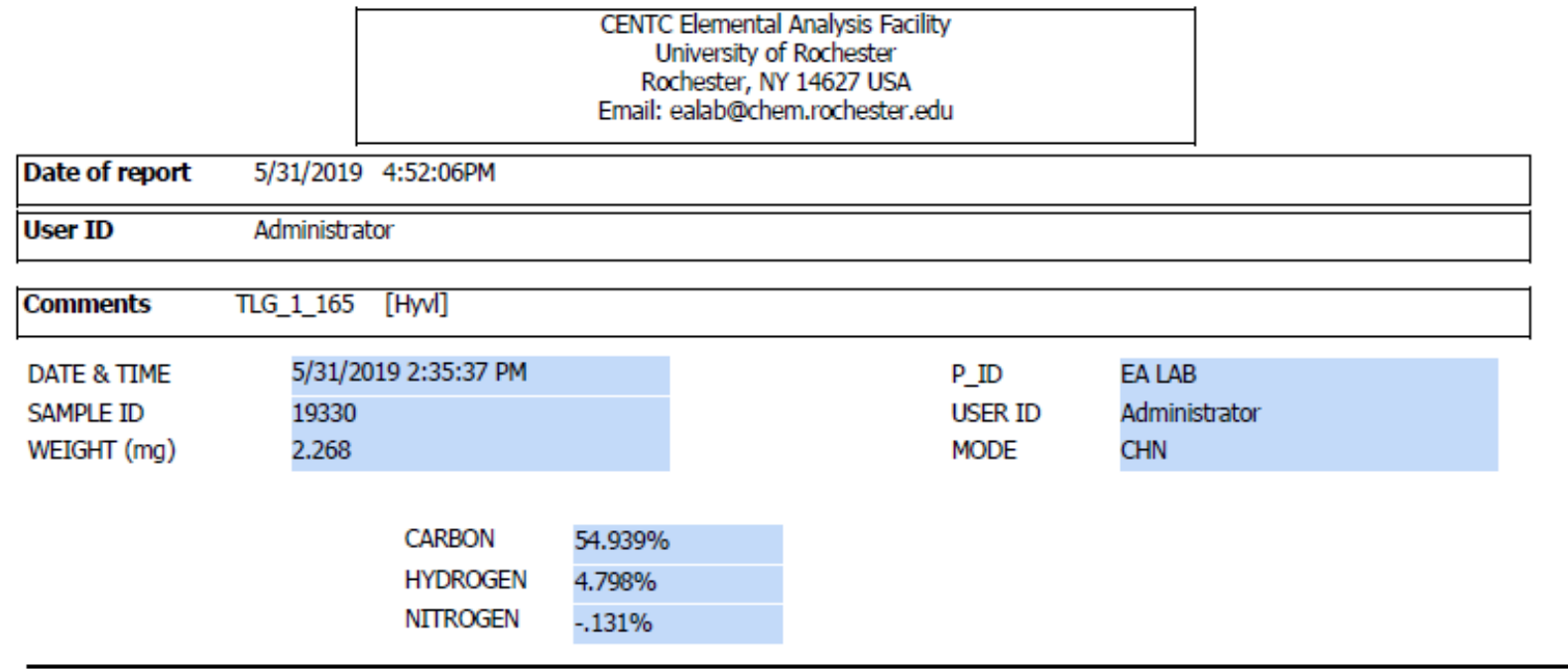

Acknowledgment

Analytical data were obtained from the CENTC Elemental Analysis Facility at the University of Rochester, funded by NSF CHE-0650456.

\section{Instrumentation}

Microanalysis samples were weighed with a PerkinElmer Model AD6000 Autobalance and their compositions were determined with a PerkinElmer 2400 Series II Analyzer.

Figure S36. EA report for $\mathbf{1 f}$ 


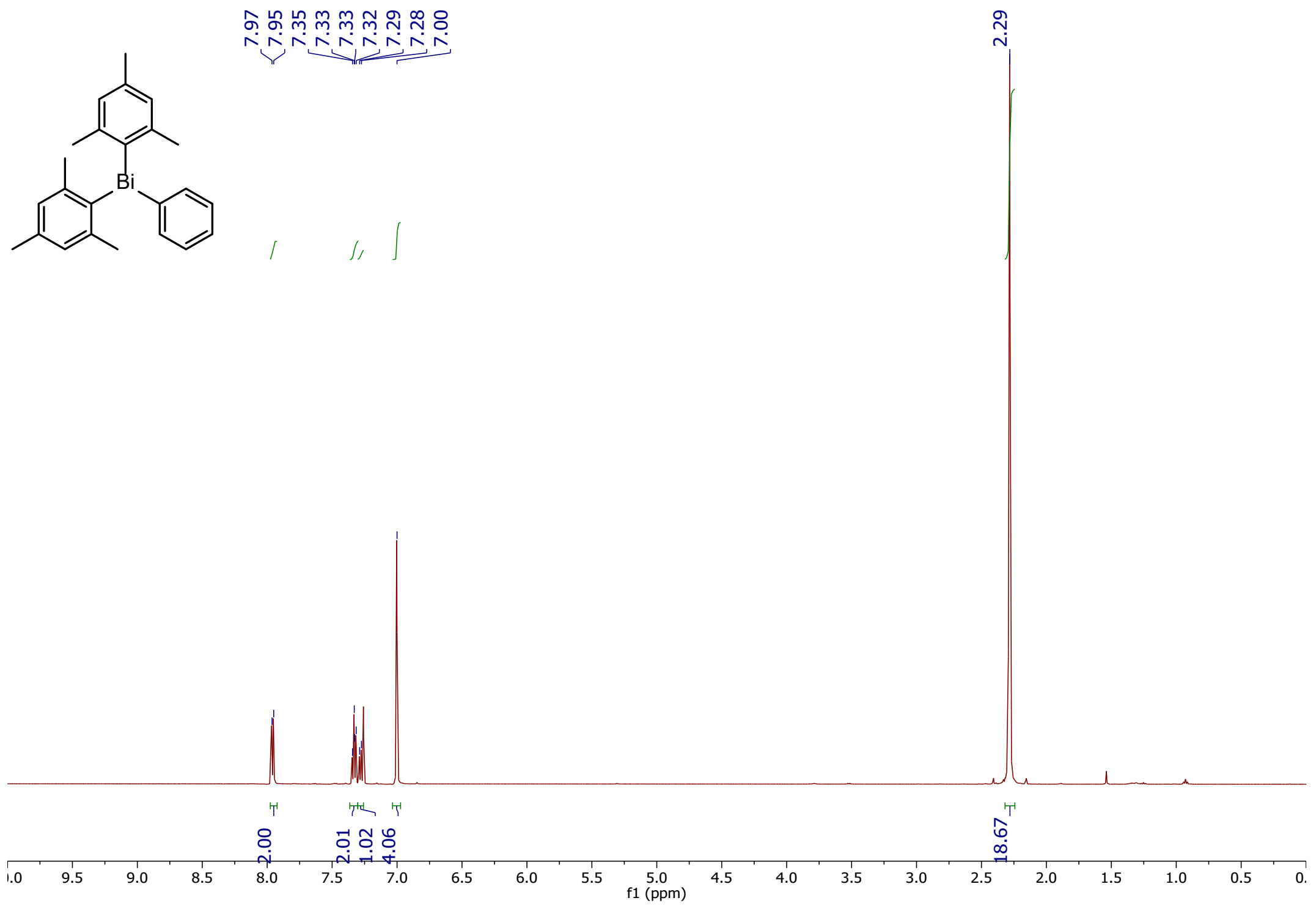

Figure S37. ${ }^{1} \mathrm{H} \mathrm{NMR}$ of $\mathbf{1 f}$ in $\mathrm{CDCl}_{3}$

S49 


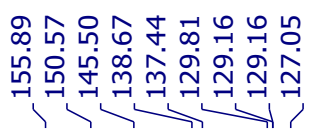

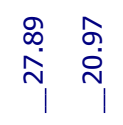<smiles>Cc1cc(C)c2c(c1)Cc1cc(C)cc(C)c1-c1c(C)cc(C)cc1B2c1ccccc1</smiles>

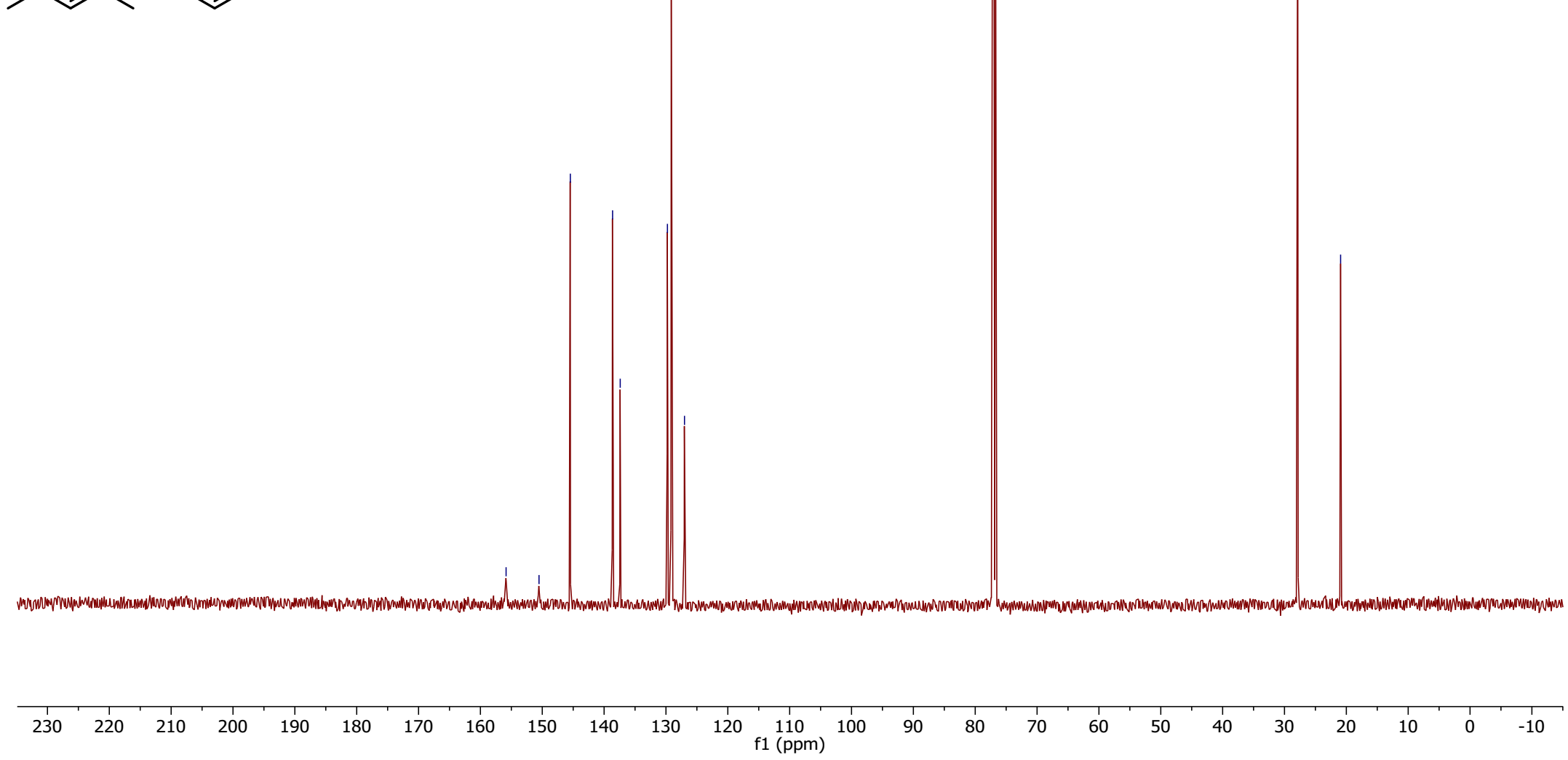

Figure S38. ${ }^{13} \mathrm{C}\left\{{ }^{1} \mathrm{H}\right\}$ NMR of $\mathbf{1 f}$ in $\mathrm{CDCl}_{3}$

S50 

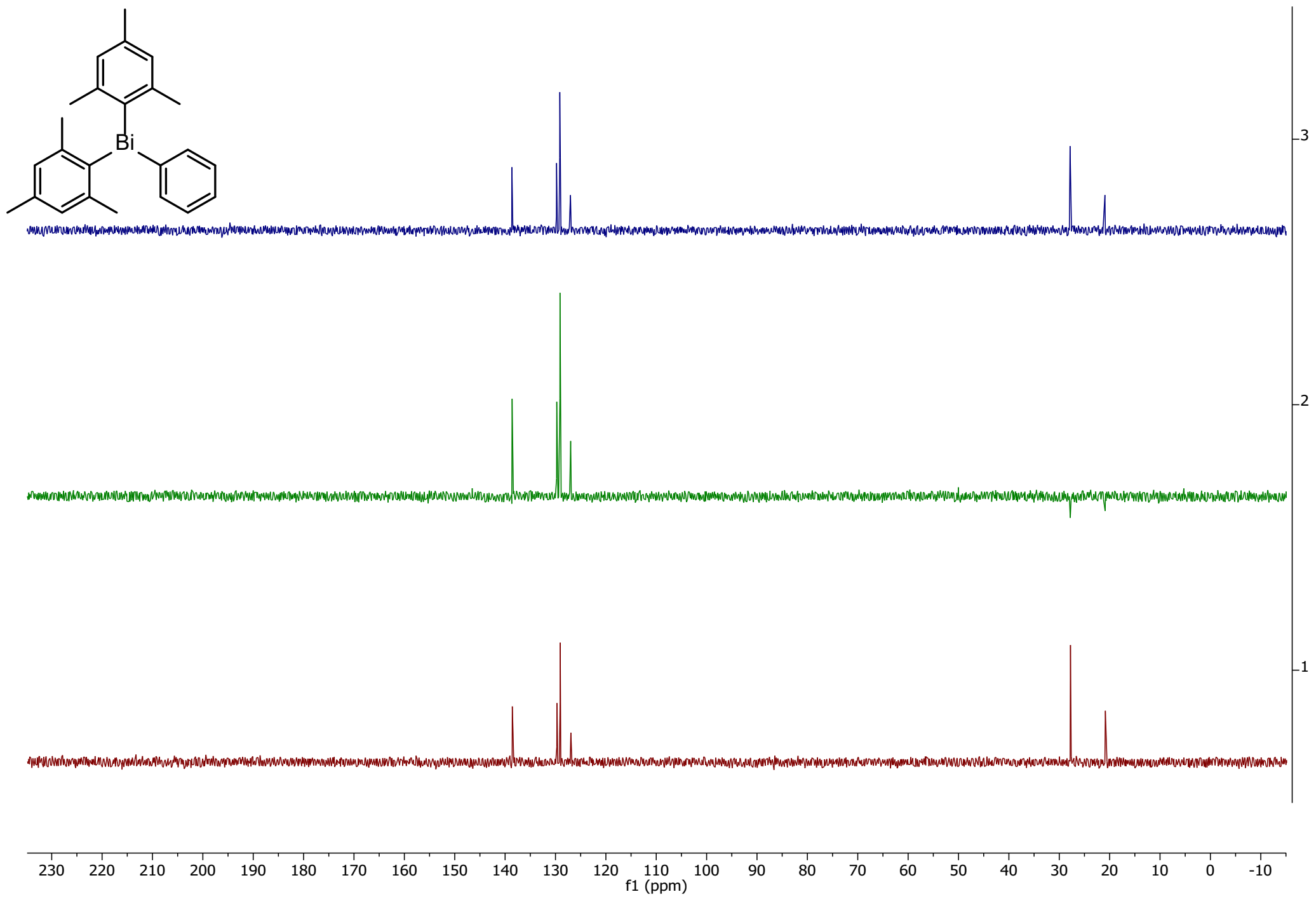

Figure S39. ${ }^{13} \mathrm{C}$ DEPT of $\mathbf{1 f}$ in $\mathrm{CDCl}_{3}$

S51 


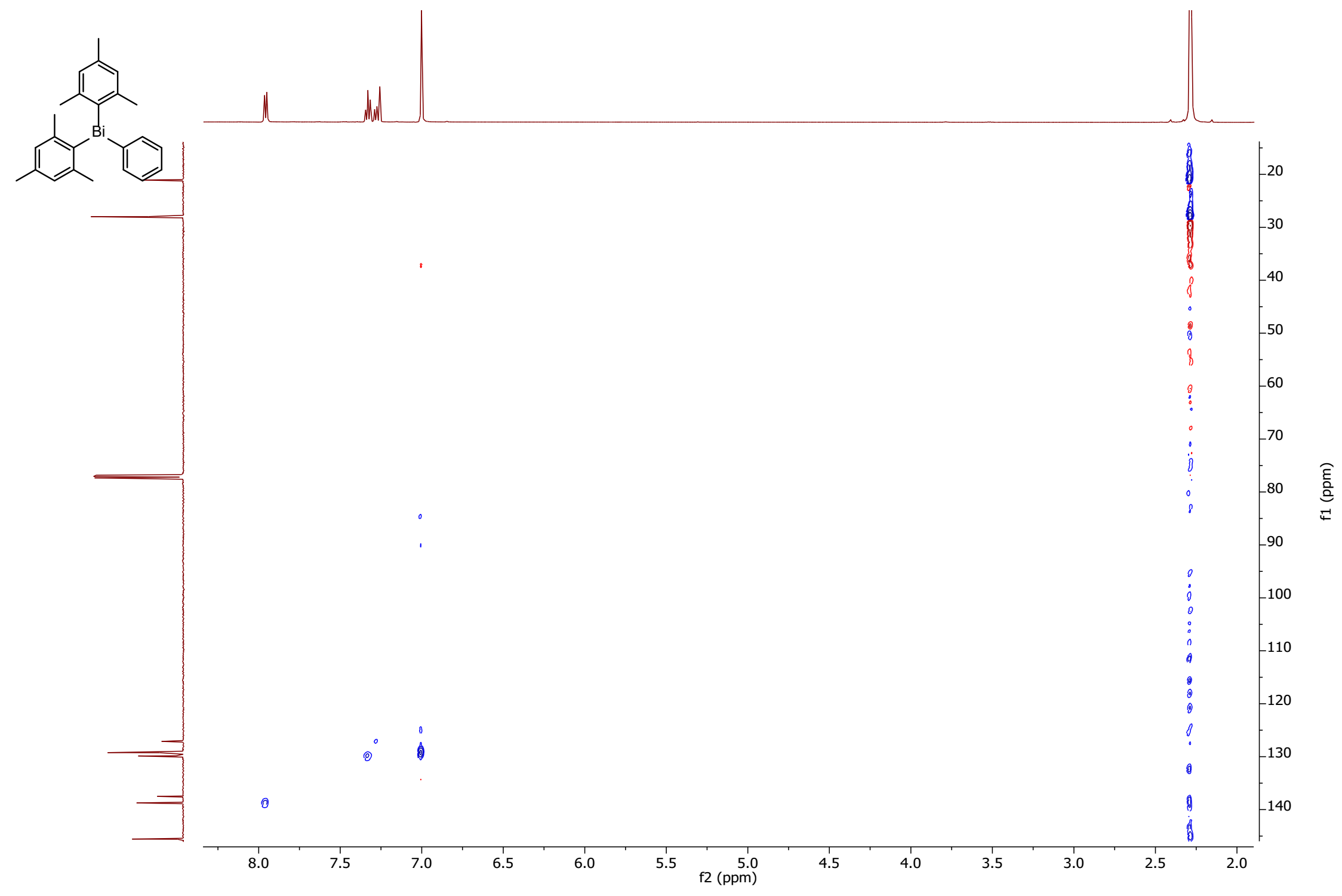

Figure S40. 2D HSQC of $\mathbf{1 f}$ in $\mathrm{CDCl}_{3}$ 


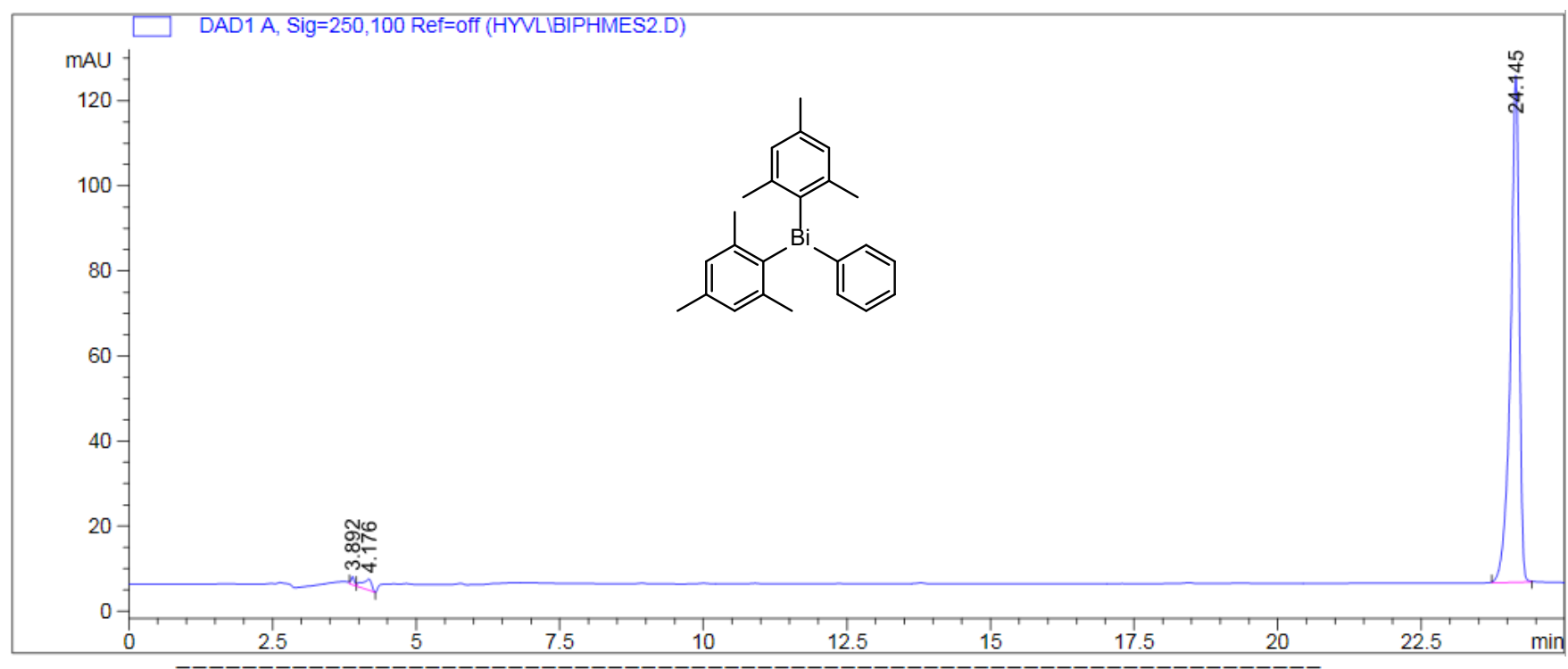

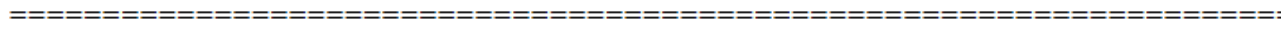

Area Percent Report

\begin{tabular}{|c|c|c|c|c|}
\hline Sorted By & : & Signal & & \multirow{5}{*}{ /ul] } \\
\hline Multiplier: & & : & 1.0000 & \\
\hline Dilution: & & : & 1.0000 & \\
\hline Sample Amount: & & : & .00000 & \\
\hline Use Multiplier \& & ilution & Factor with & ISTDS & \\
\hline \multicolumn{5}{|c|}{ Signal 1: DAD1 A, Sig=250,100 Ref=off } \\
\hline $\begin{array}{l}\text { Peak RetTime Type } \\
\# \quad[\mathrm{~min}]\end{array}$ & $\begin{array}{l}\text { Width } \\
\text { [min] }\end{array}$ & $\begin{array}{c}\text { Area } \\
{\left[\mathrm{mAU}{ }^{*} \mathrm{~s}\right]}\end{array}$ & $\begin{array}{l}\text { Height } \\
\text { [mAU] }\end{array}$ & $\begin{array}{c}\text { Area } \\
\frac{\%}{6}\end{array}$ \\
\hline$---|-------|---$ & ------ & $---------\mid$ & -------- & ------ \\
\hline $3.892 \mathrm{BV}$ & 0.0598 & 7.99999 & 1.95155 & 0.6197 \\
\hline $24.176 \mathrm{VV}$ & 0.1464 & 27.91670 & 2.66161 & 2.1623 \\
\hline $324.145 \mathrm{BB}$ & 0.1568 & 1255.12268 & 119.23830 & 97.2180 \\
\hline
\end{tabular}

Figure S41. HPLC Chromatogram of $1 \mathrm{f}$ 


\section{NMR Spectra and EA Report of Compound $1 \mathrm{~g}$}<smiles>Cc1ccc([Bi](c2c(C)cc(C)cc2C)c2c(C)cc(C)cc2C#N)c(C)c1</smiles>

Chemical Formula: $\mathrm{BiC}_{25} \mathrm{H}_{26} \mathrm{~N}$

Molecular Weight: 549.47

Elemental Analysis: C: 54.65; Bi: 38.03; H: 4.77; N:2.35

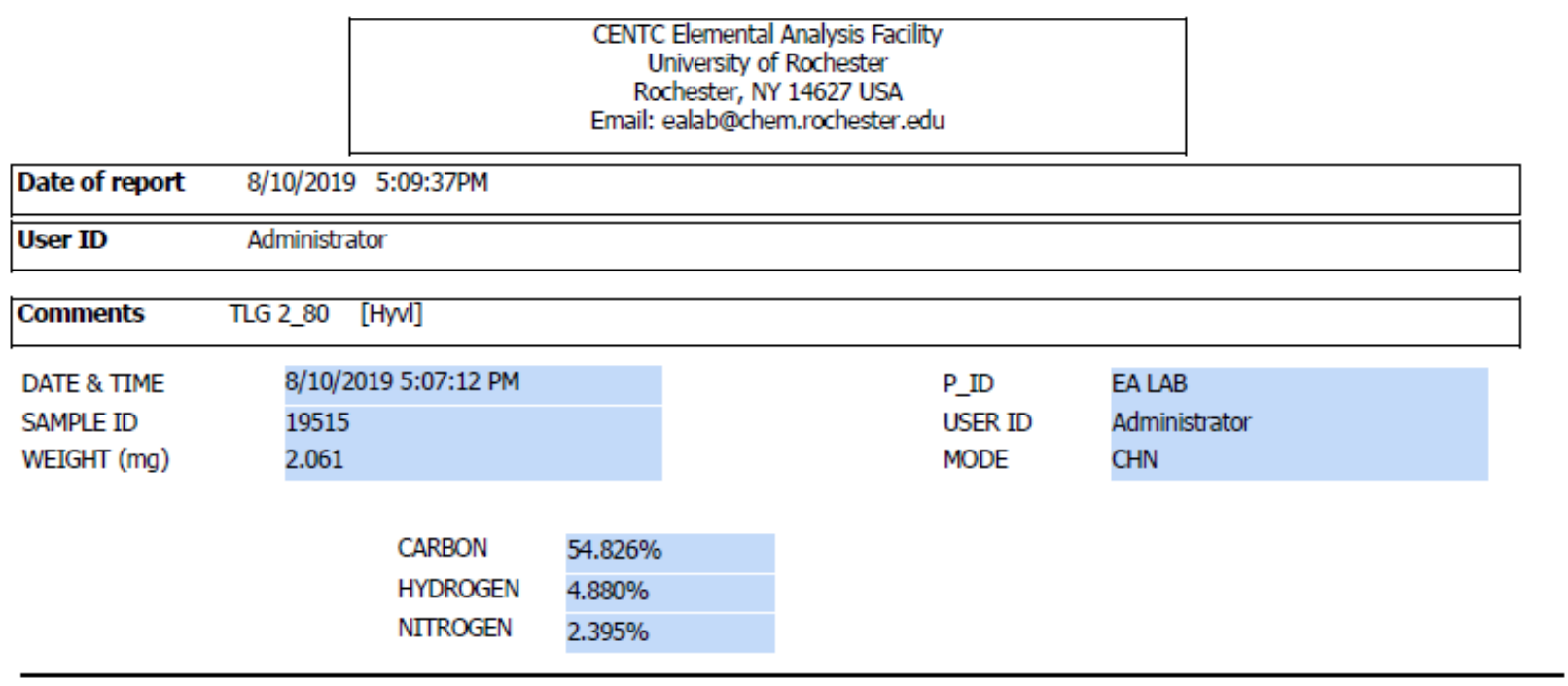

\section{Acknowledgment}

Analytical data were obtained from the CENTC Elemental Analysis Facility at the University of Rochester, funded by NSF CHE-0650456.

\section{Instrumentation}

Microanalysis samples were weighed with a PerkinElmer Model AD6000 Autobalance and their compositions were determined with a PerkinElmer 2400 Series II Analyzer.

Figure $\mathbf{S 4 2}$. EA report for $\mathbf{1 g}$ 

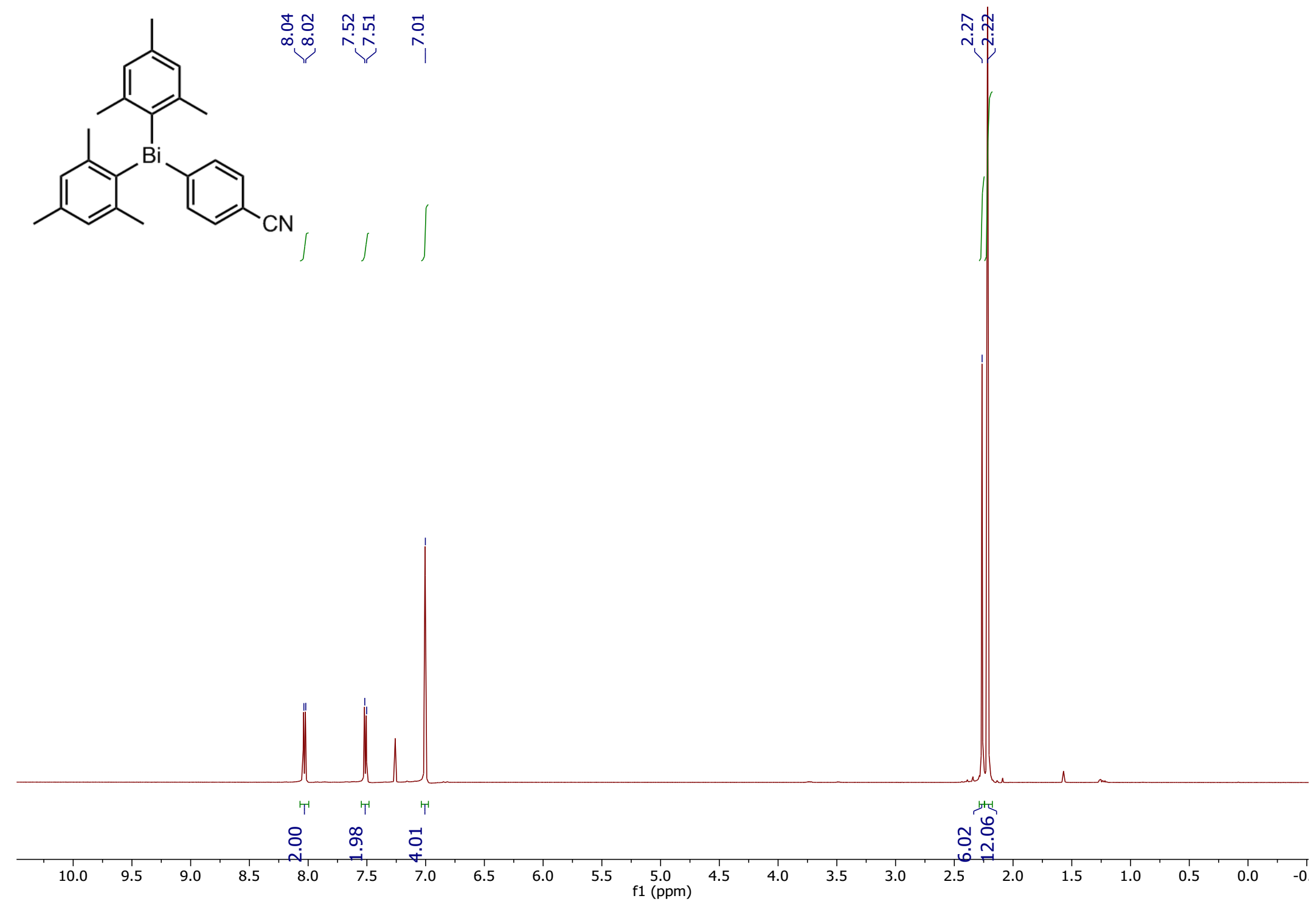

Figure S43. ${ }^{1} \mathrm{H}$ NMR of $1 \mathrm{~g}$ in $\mathrm{CDCl}_{3}$ 

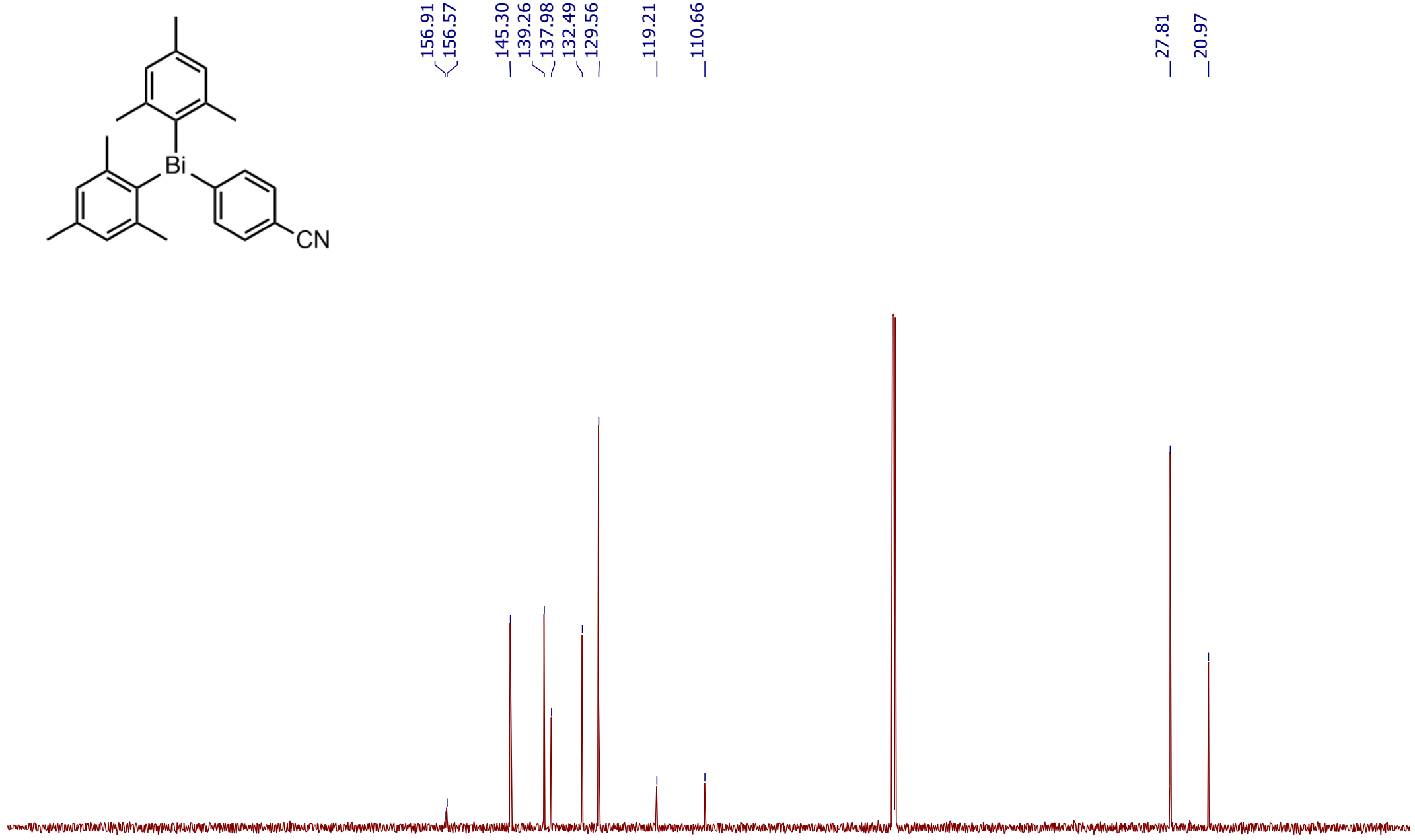

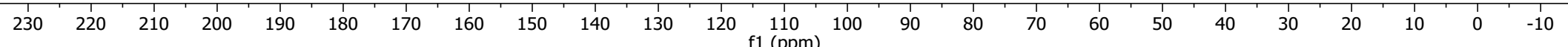

Figure S44. ${ }^{13} \mathrm{C}\left\{{ }^{1} \mathrm{H}\right\}$ NMR of $1 \mathrm{~g}$ in $\mathrm{CDCl}_{3}$ 

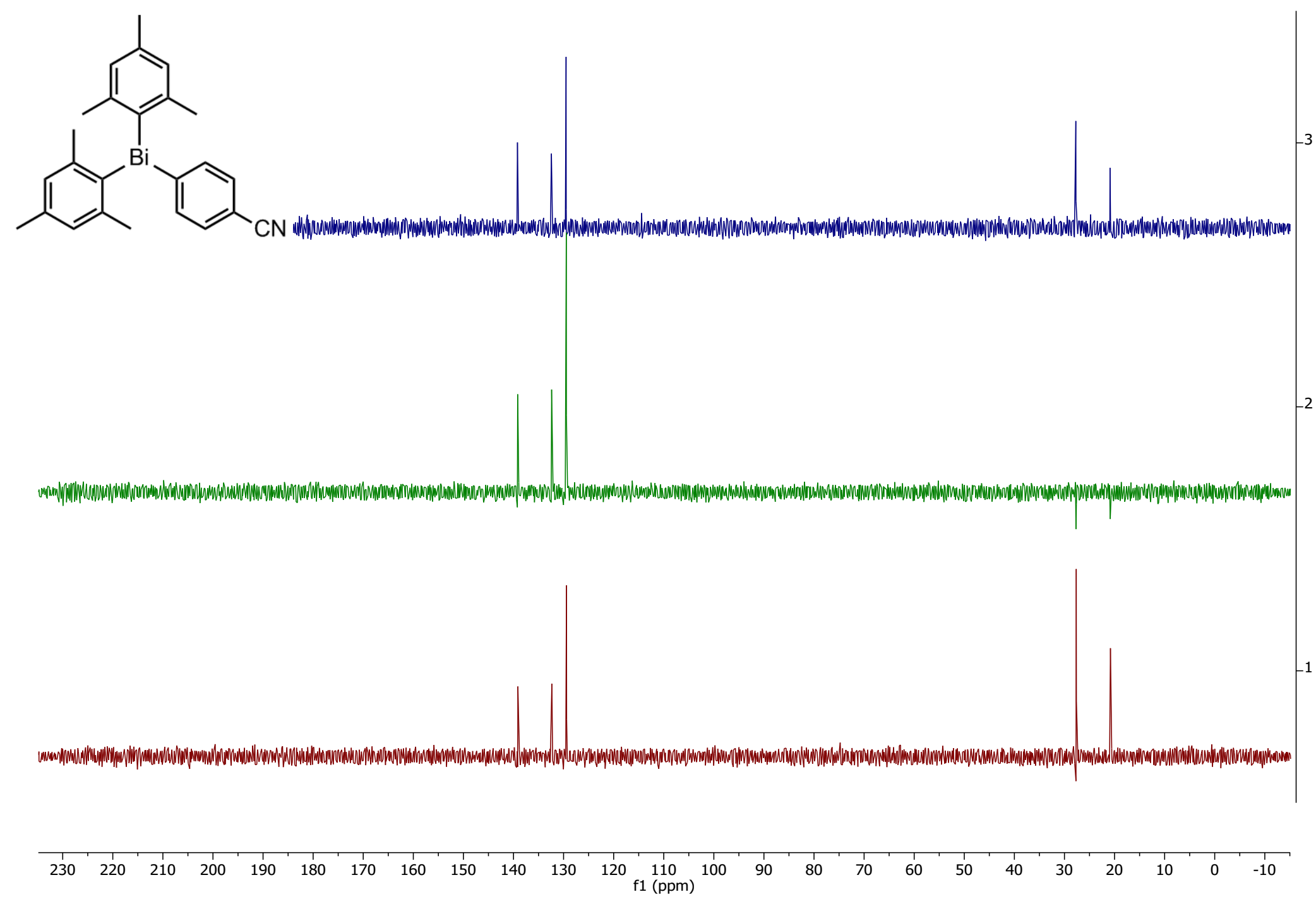

Figure S45. ${ }^{13} \mathrm{C}$ DEPT of $1 \mathrm{~g}$ in $\mathrm{CDCl}_{3}$ 


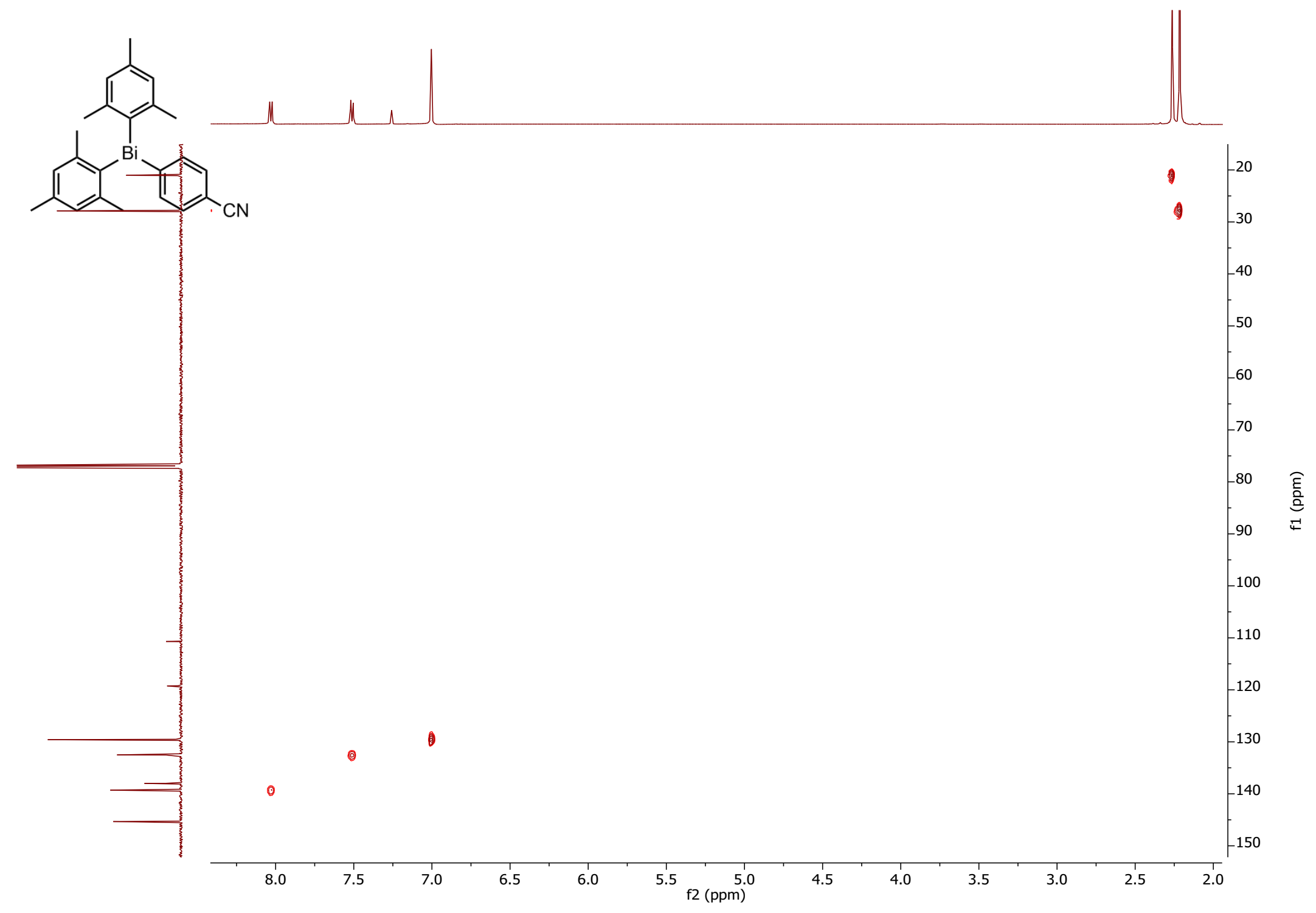

Figure S46. 2D HSQC of $1 \mathrm{~g}$ in $\mathrm{CDCl}_{3}$ 


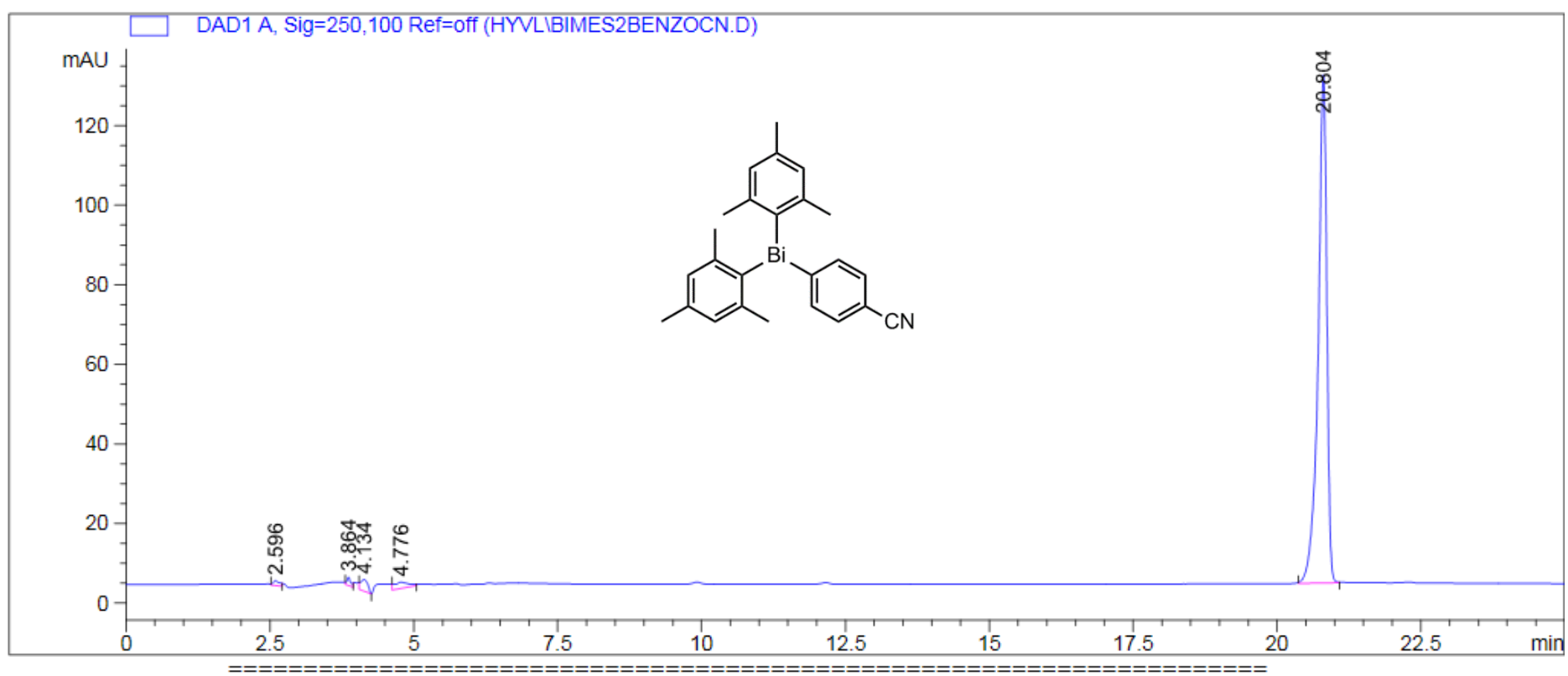

Area Percent Report

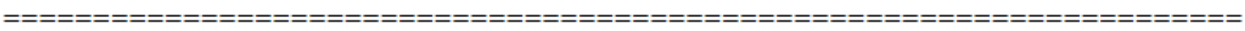

$\begin{array}{lcccc}\text { Sorted By } & : & \text { Signal } & & \\ \text { Multiplier: } & : & 1.0000 & \\ \text { Dilution: } & : & 1.0000 & \\ \text { Sample Amount: } & : & 20.00000 & \text { [ng/ul] } & \text { (not used in calc.) } \\ \text { Use Multiplier \& Dilution Factor with ISTDs } & \end{array}$

Use Multiplier \& Dilution Factor with ISTDs

Signal 1: DAD1 A, Sig=250,100 Ref=off

\begin{tabular}{|c|c|c|c|c|c|c|}
\hline $\begin{array}{c}\text { Peak } \\
\text { \# }\end{array}$ & $\begin{array}{l}\text { RetTime } \\
\text { [min] }\end{array}$ & Type & $\begin{array}{l}\text { Width } \\
\text { [min] }\end{array}$ & $\begin{array}{c}\text { Area } \\
{\left[\mathrm{mAU} U^{*} \mathrm{~S}\right]}\end{array}$ & $\begin{array}{l}\text { Height } \\
\text { [mAU] }\end{array}$ & $\begin{array}{c}\text { Area } \\
\frac{\circ}{0}\end{array}$ \\
\hline & -- & & ---- & ---------- & ------ & $------\mid$ \\
\hline 1 & 2.596 & $\mathrm{BB}$ & 0.1066 & 9.04404 & 1.16519 & 0.6390 \\
\hline 2 & 3.864 & $\mathrm{BB}$ & 0.0664 & 9.94713 & 2.13506 & 0.7029 \\
\hline 3 & 4.134 & BV & 0.1346 & 24.92050 & 2.94877 & 1.7609 \\
\hline 4 & 4.776 & $\mathrm{BB}$ & 0.2193 & 27.37470 & 1.60261 & 1.9343 \\
\hline 5 & 20.804 & $\mathrm{BB}$ & 0.1566 & 1343.94800 & 127.86018 & 94.9629 \\
\hline
\end{tabular}

Figure S47. HPLC Chromatogram of 19 


\section{NMR Spectra and EA Report of Compound $1 \mathrm{~h}$}<smiles>COc1ccc([BH-]c2c(C)cc(C)cc2C)cc1C</smiles>

Chemical Formula: $\mathrm{BiOC}_{25} \mathrm{H}_{29}$

Molecular Weight: 554.49

Elemental Analysis: C: 54.15; Bi: 37.69; H: 5.27; O: 2.89

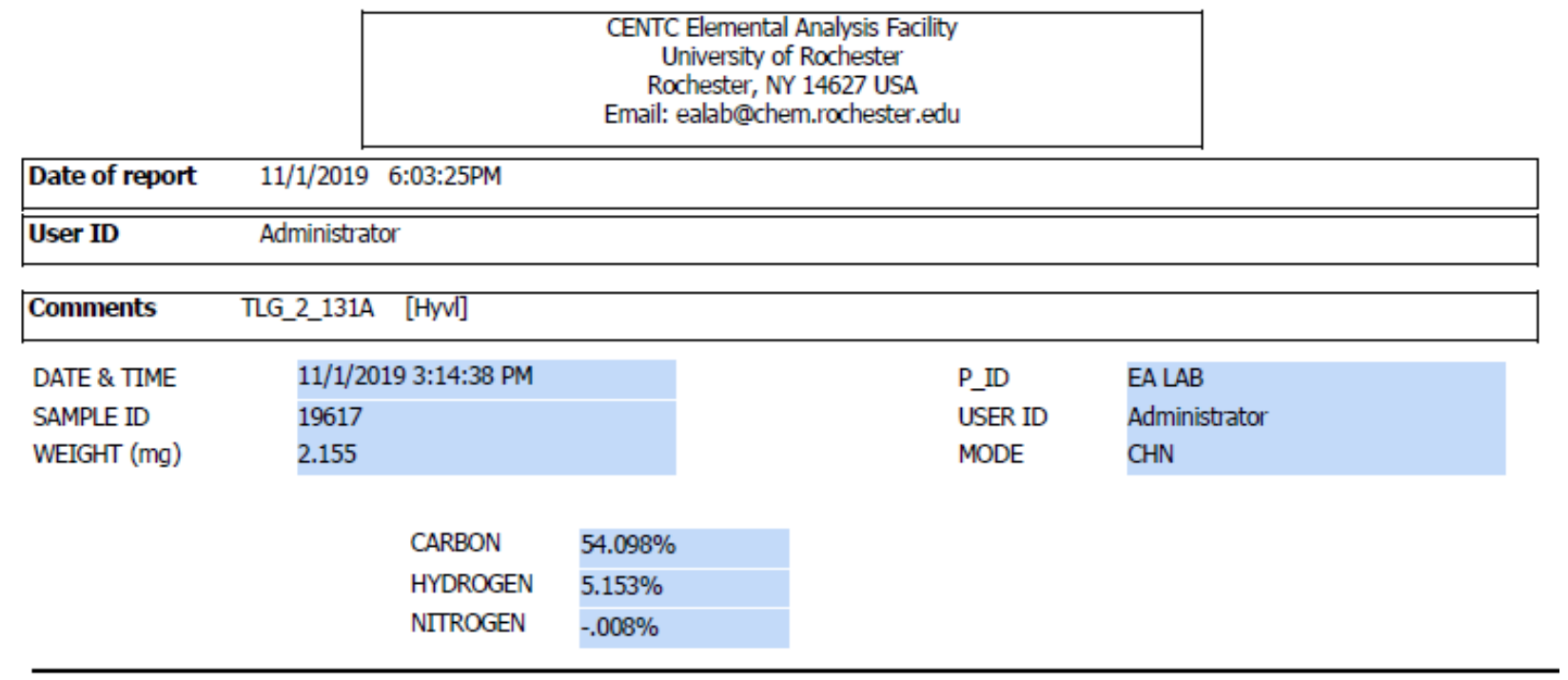

\section{Acknowledgment}

Analytical data were obtained from the CENTC Elemental Analysis Facility at the University of Rochester, funded by NSF CHE- 0650456 .

\section{Instrumentation}

Microanalysis samples were weighed with a PerkinElmer Model AD6000 Autobalance and their compositions were determined with a PerkinElmer 2400 Series II Analyzer.

Figure S48. EA report for $\mathbf{1 h}$ 


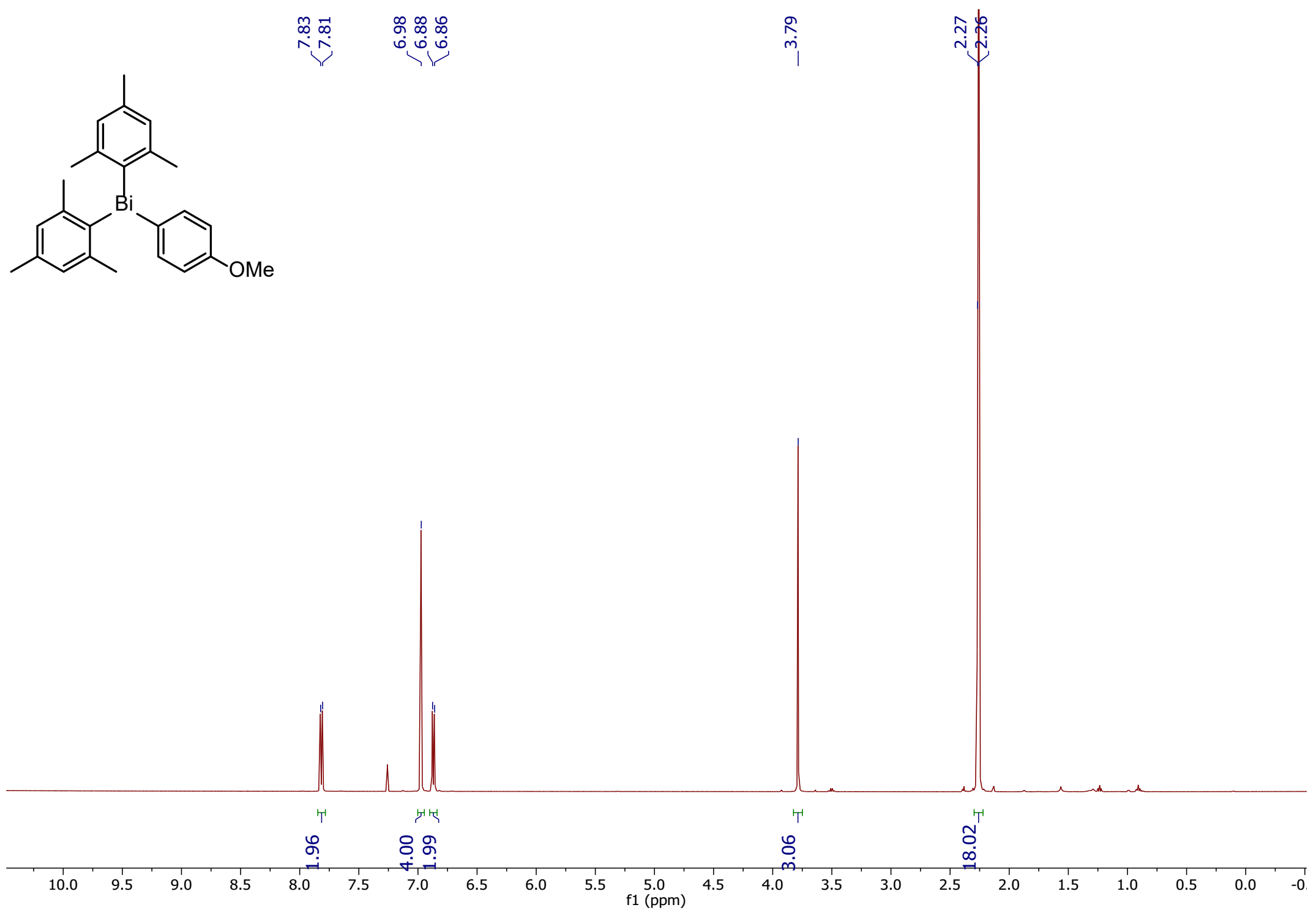

Figure S49. ${ }^{1} \mathrm{H} \mathrm{NMR}$ of $\mathbf{1 h}$ in $\mathrm{CDCl}_{3}$

S61 


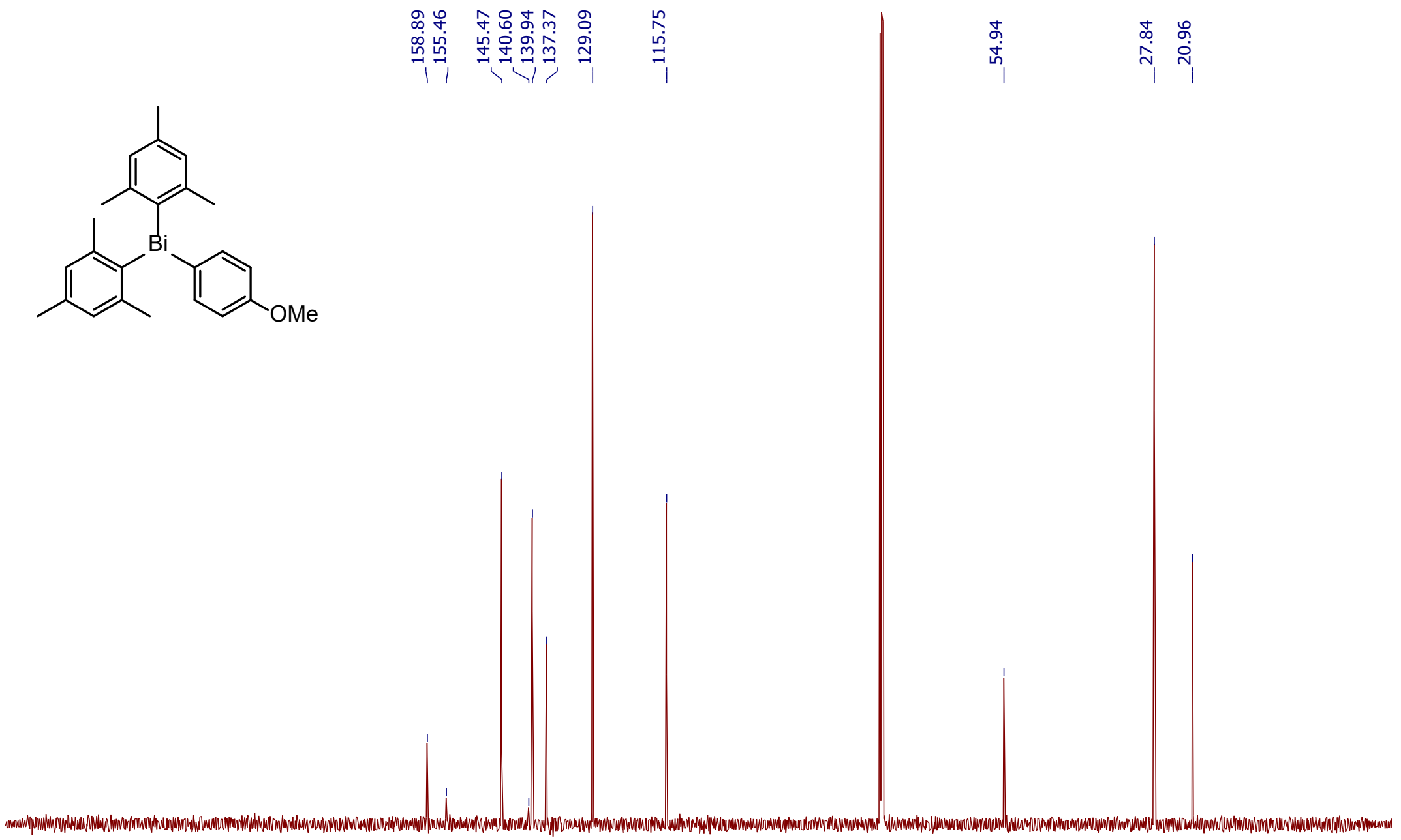

$\begin{array}{llllllllllllllllllllllllllllll}230 & 220 & 210 & 200 & 190 & 180 & 170 & 160 & 150 & 140 & 130 & 120 & 110 & 100 & 90 & 80 & 70 & 60 & 50 & 40 & 30 & 20 & 10 & 0 & -10\end{array}$

Figure S50. ${ }^{13} \mathrm{C}\left\{{ }^{1} \mathrm{H}\right\}$ NMR of $\mathbf{1 h}$ in $\mathrm{CDCl}_{3}$ 


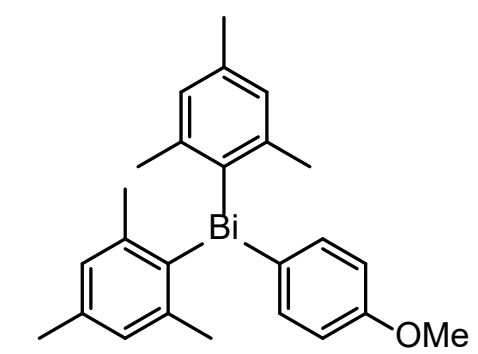

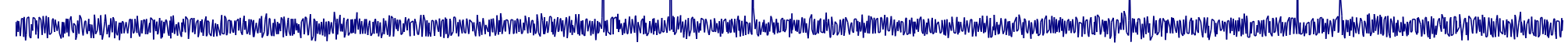

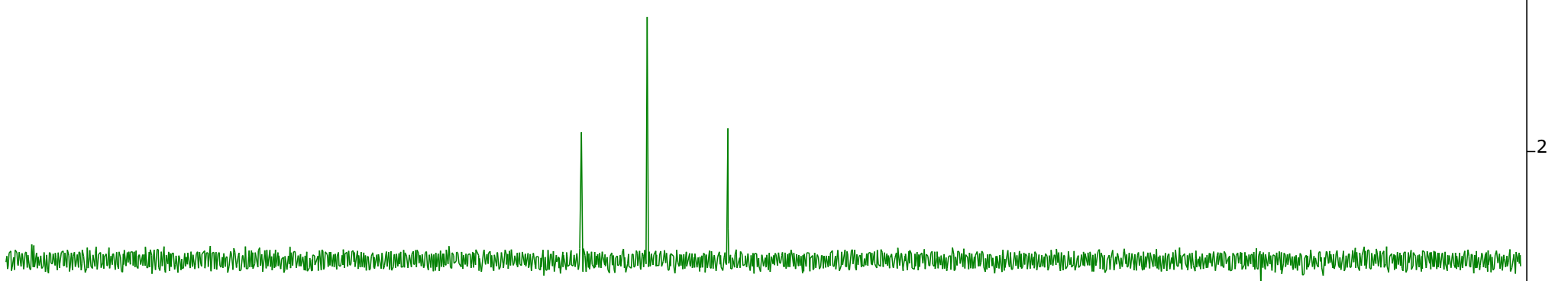

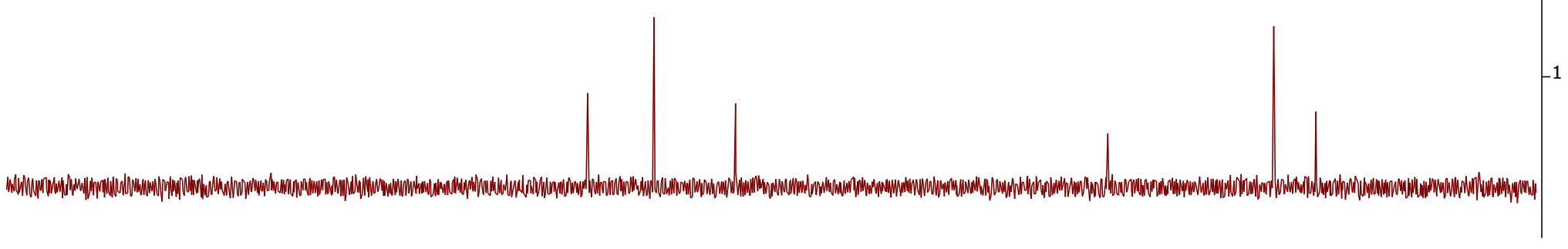

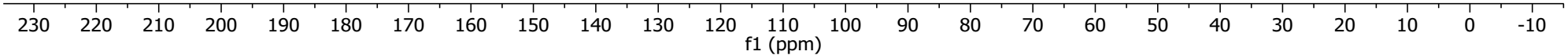

Figure S51. ${ }^{13} \mathrm{C}$ DEPT of $\mathbf{1 h}$ in $\mathrm{CDCl}_{3}$ 


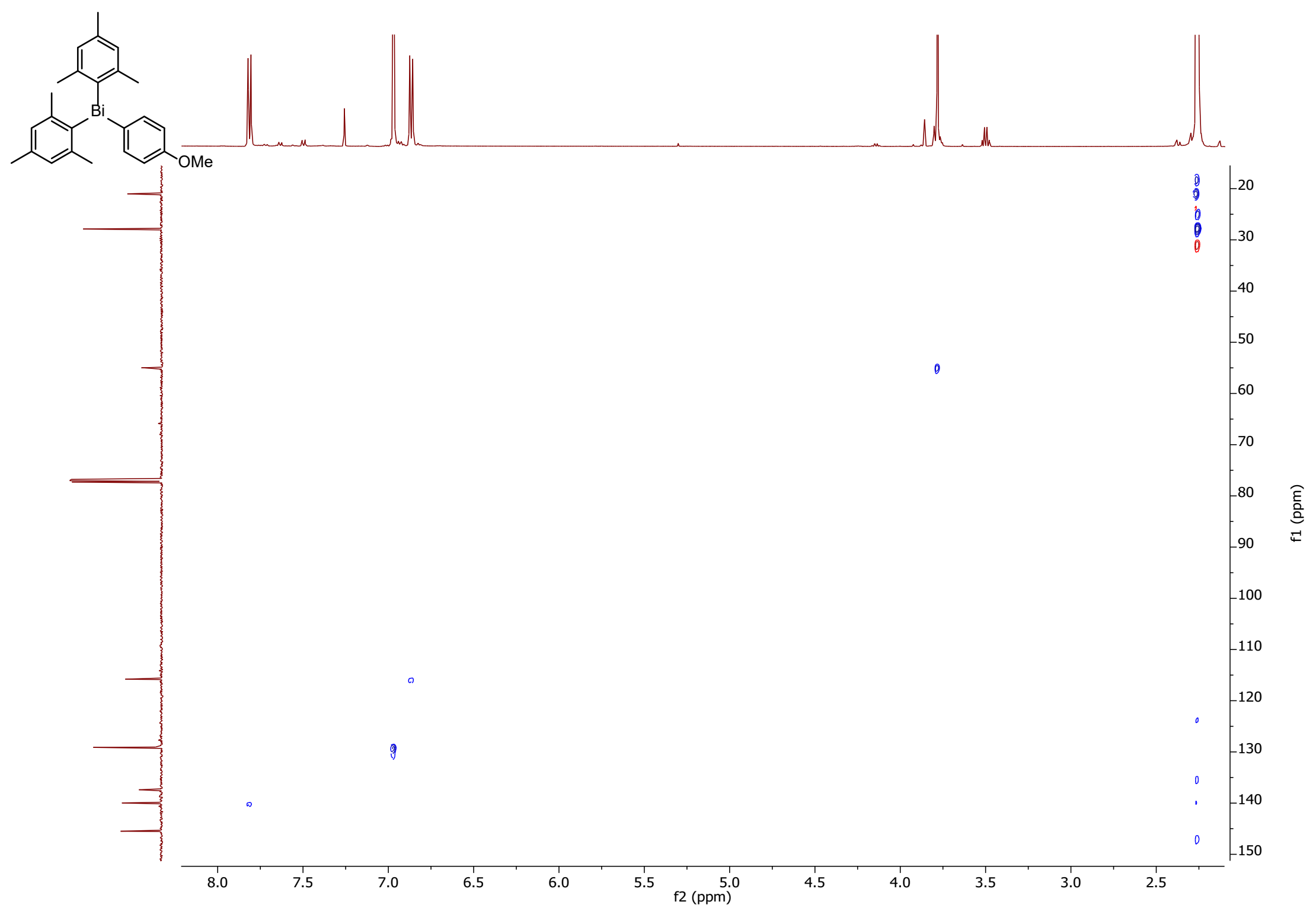

Figure S52. 2D HSQC of $\mathbf{1 h}$ in $\mathrm{CDCl}_{3}$ 


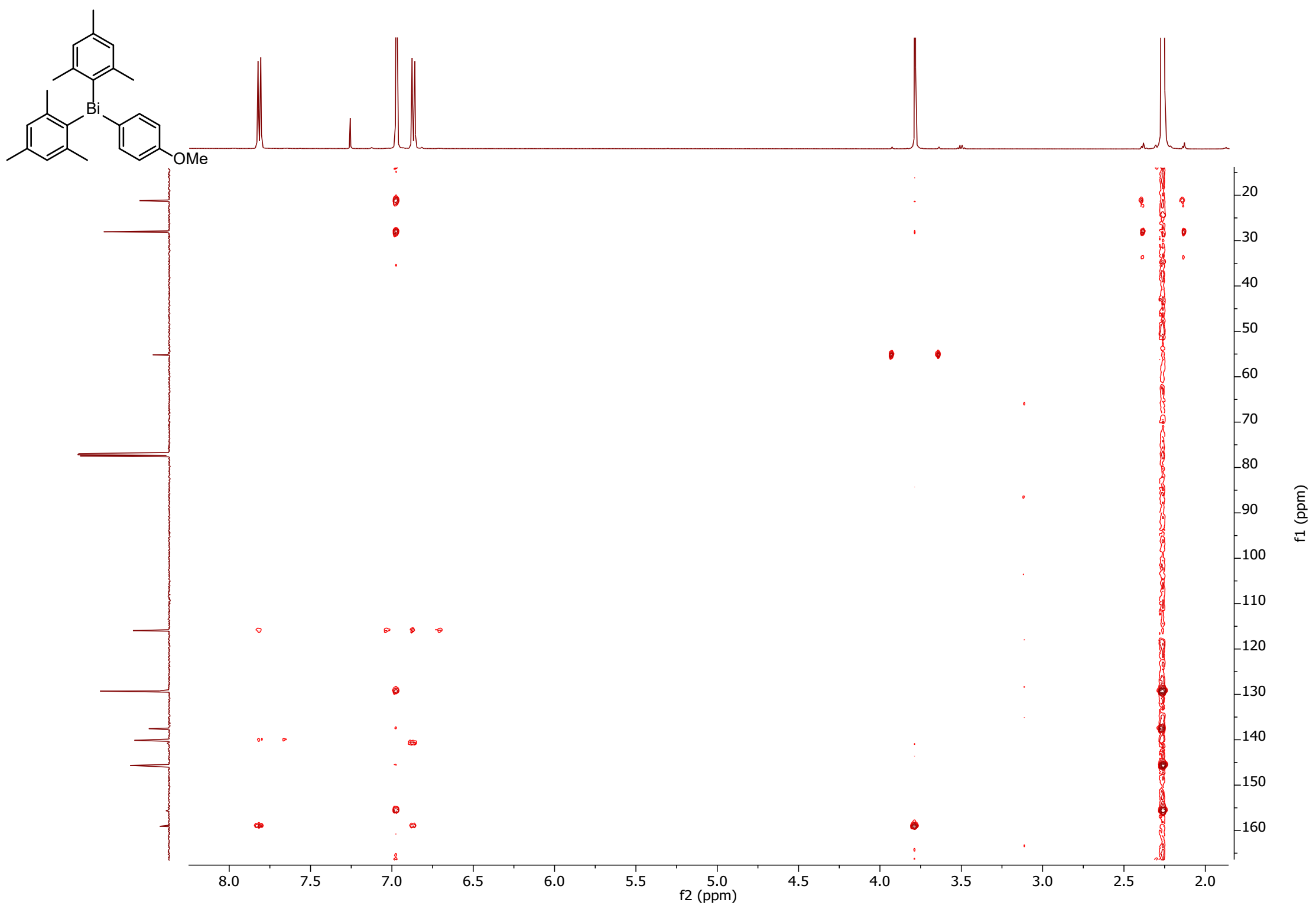

Figure S53. 2D HMBC of $\mathbf{1 h}$ in $\mathrm{CDCl}_{3}$ 


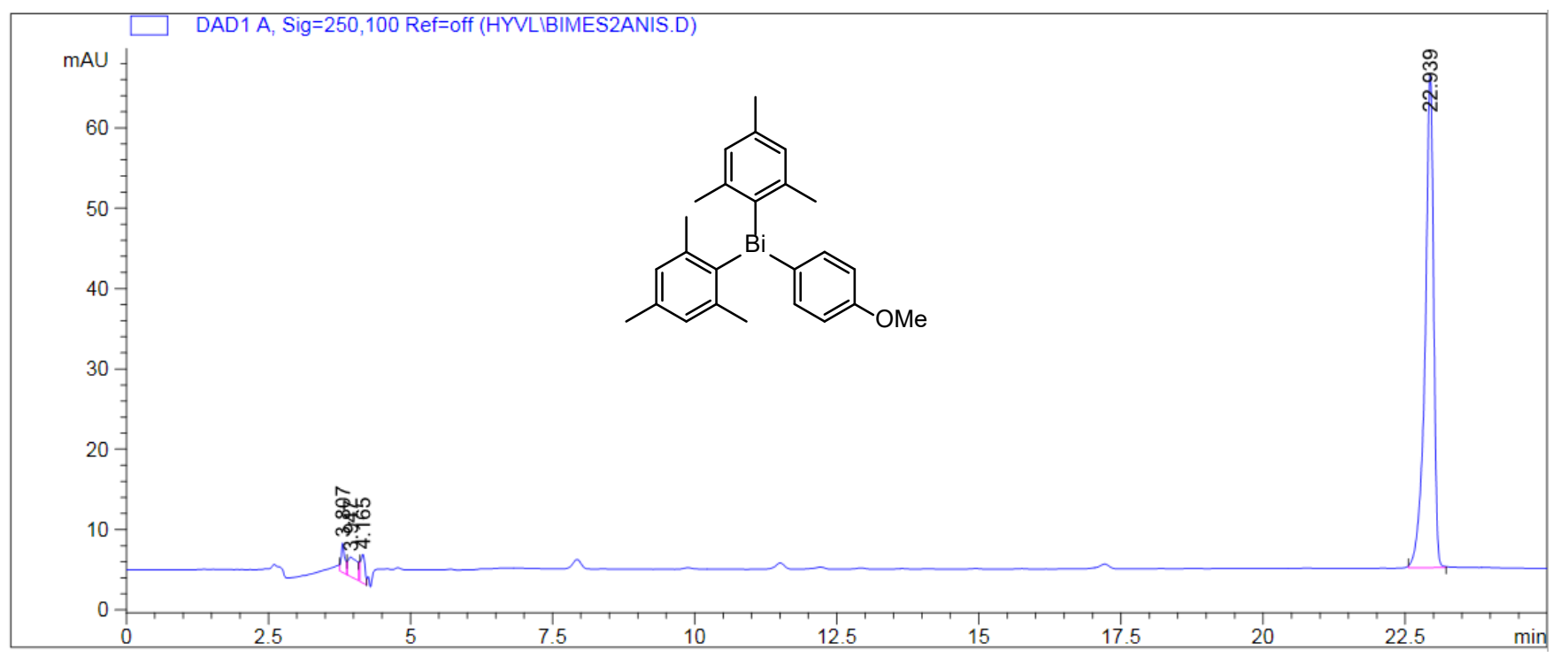

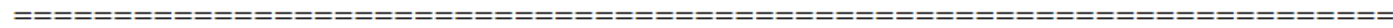

Area Percent Report

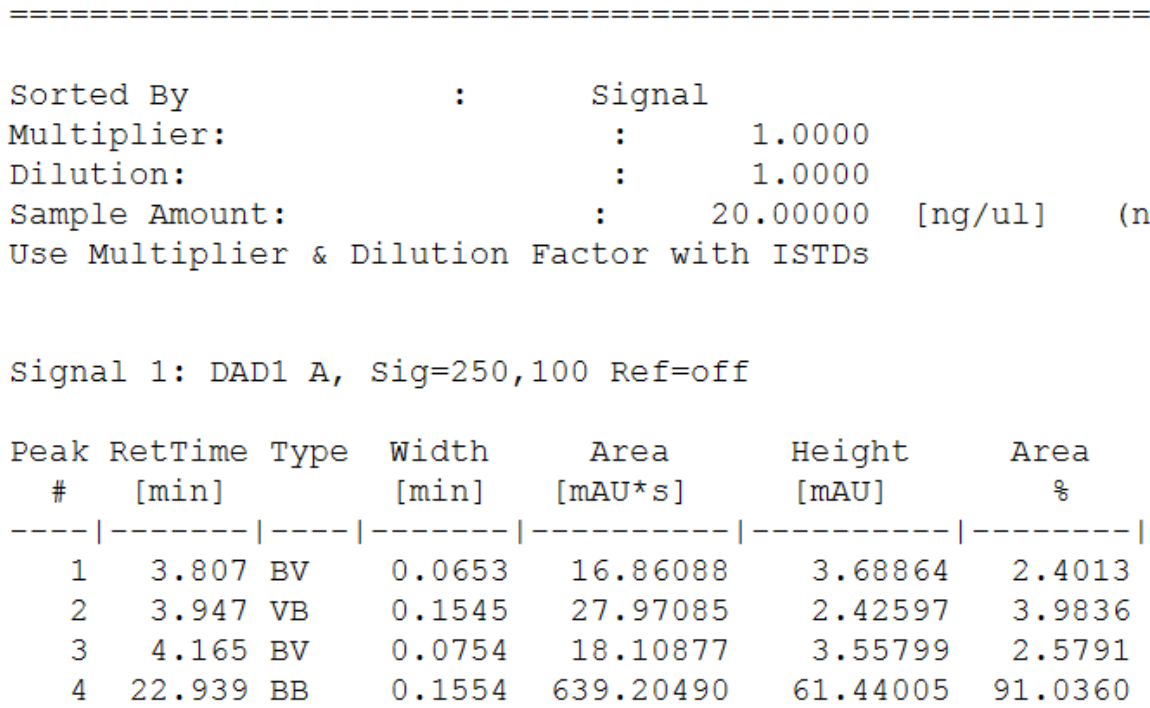

Figure S54. HPLC Chromatogram of $\mathbf{1 h}$ 


\section{NMR Spectra and EA Report of Compound 1i}<smiles>N#Cc1ccc([Bi](c2ccccc2)c2ccc(C#N)cc2)cc1</smiles>

Chemical Formula: $\mathrm{BiN}_{2} \mathrm{C}_{20} \mathrm{H}_{13}$

Molecular Weight: 490.32

Elemental Analysis: C: 48.99; Bi: 42.62; H: 2.67; N: 5.71

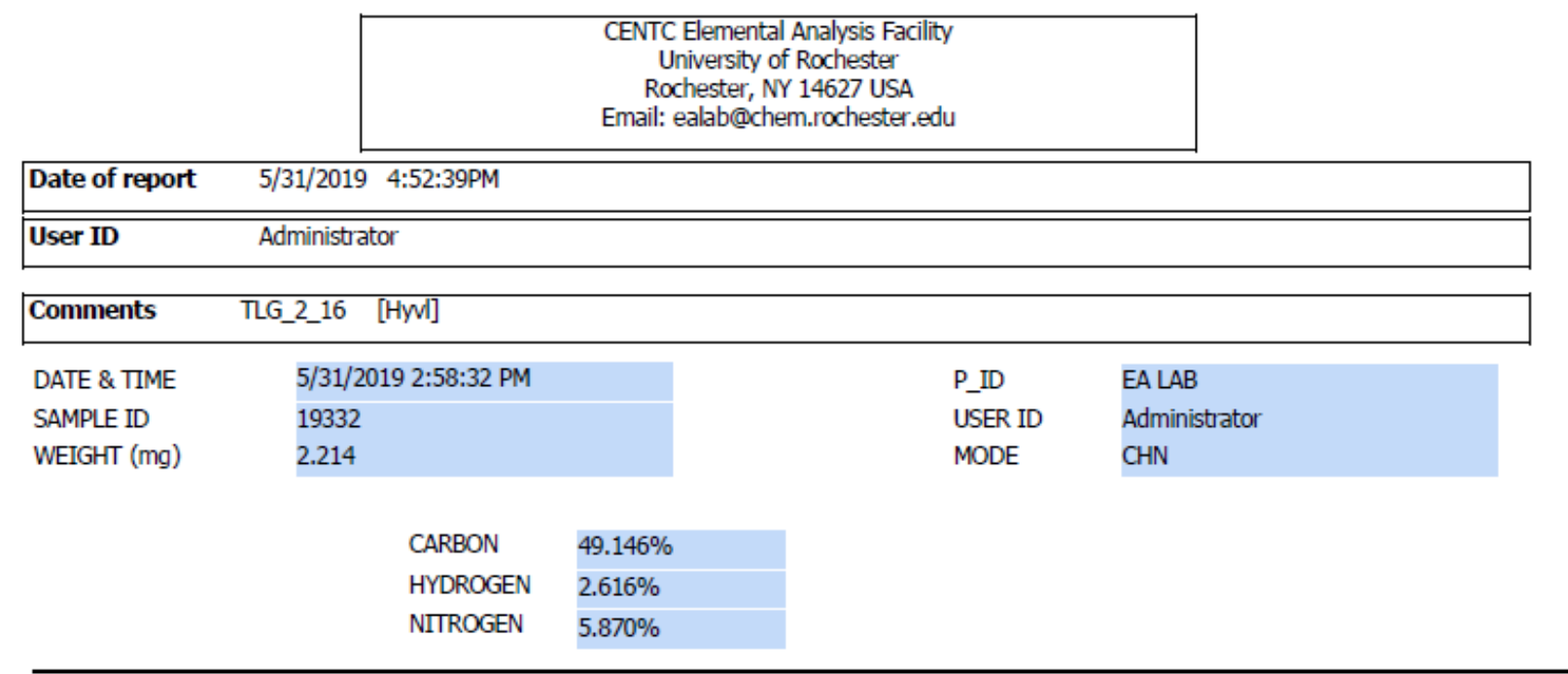

\section{Acknowledgment}

Analytical data were obtained from the CENTC Elemental Analysis Facility at the University of Rochester, funded by NSF CHE-0650456.

\section{Instrumentation}

Microanalysis samples were weighed with a PerkinElmer Model AD6000 Autobalance and their compositions were determined with a PerkinElmer 2400 Series II Analyzer.

Figure S55. EA report for $1 \mathbf{i}$ 


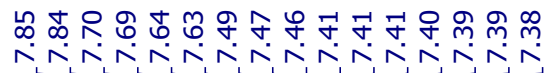
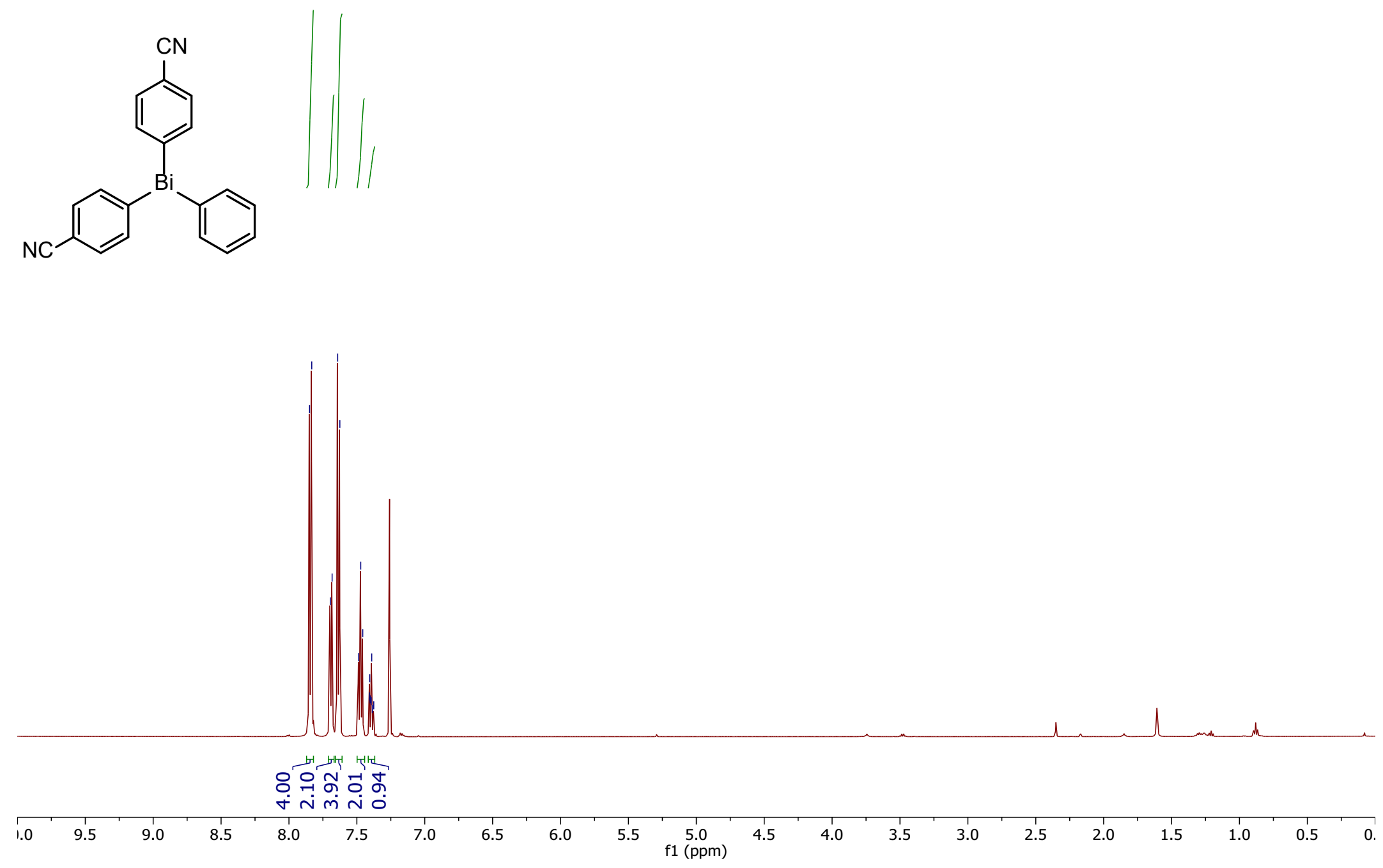

Figure S56. ${ }^{1} \mathrm{H}$ NMR of $1 \mathrm{i}$ in $\mathrm{CDCl}_{3}$ 


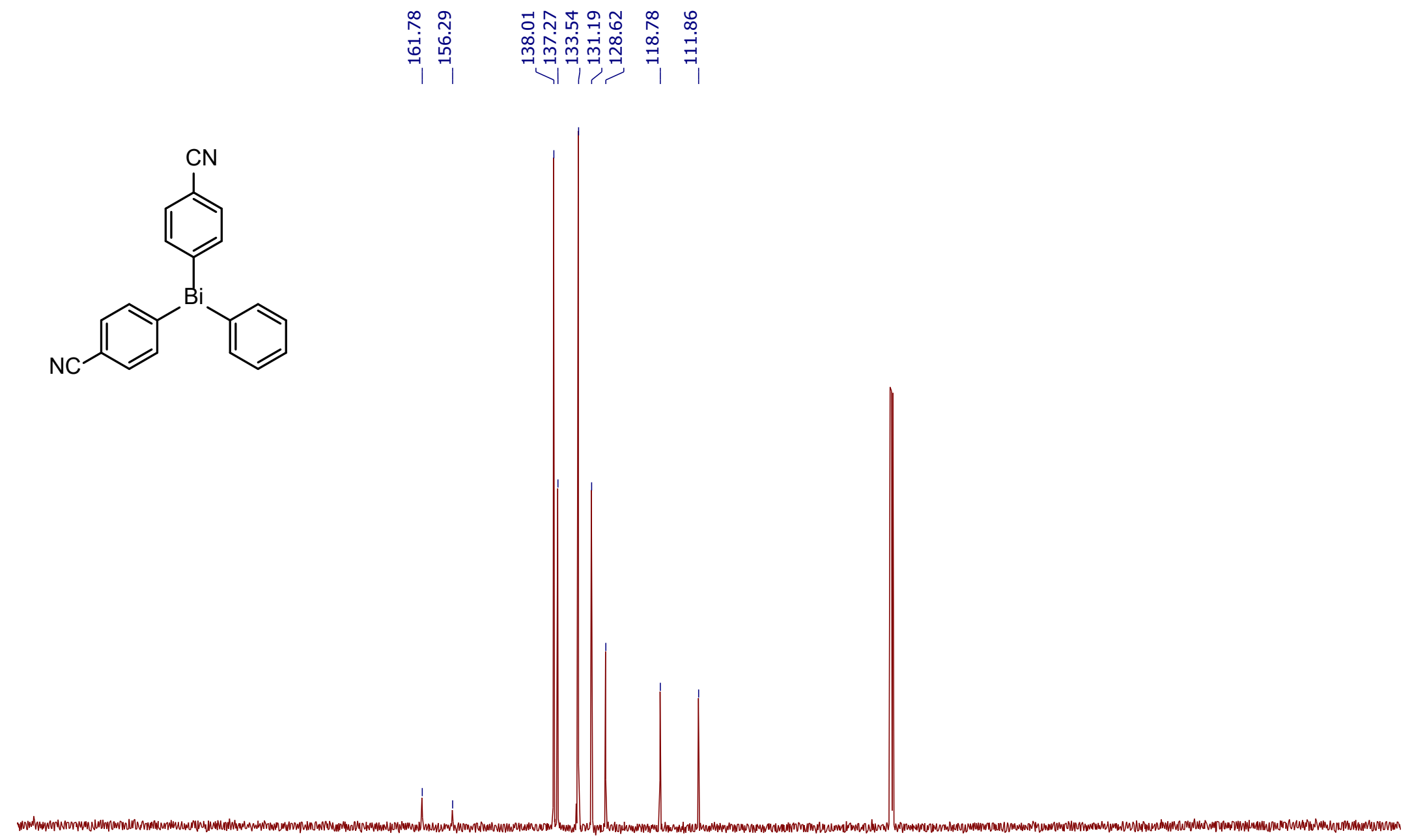

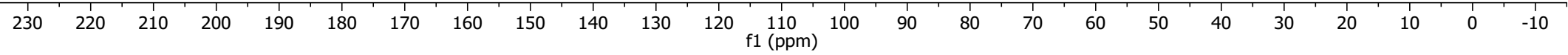

Figure S57. ${ }^{13} \mathrm{C}\left\{{ }^{1} \mathrm{H}\right\}$ NMR of $1 \mathrm{i}$ in $\mathrm{CDCl}_{3}$ 

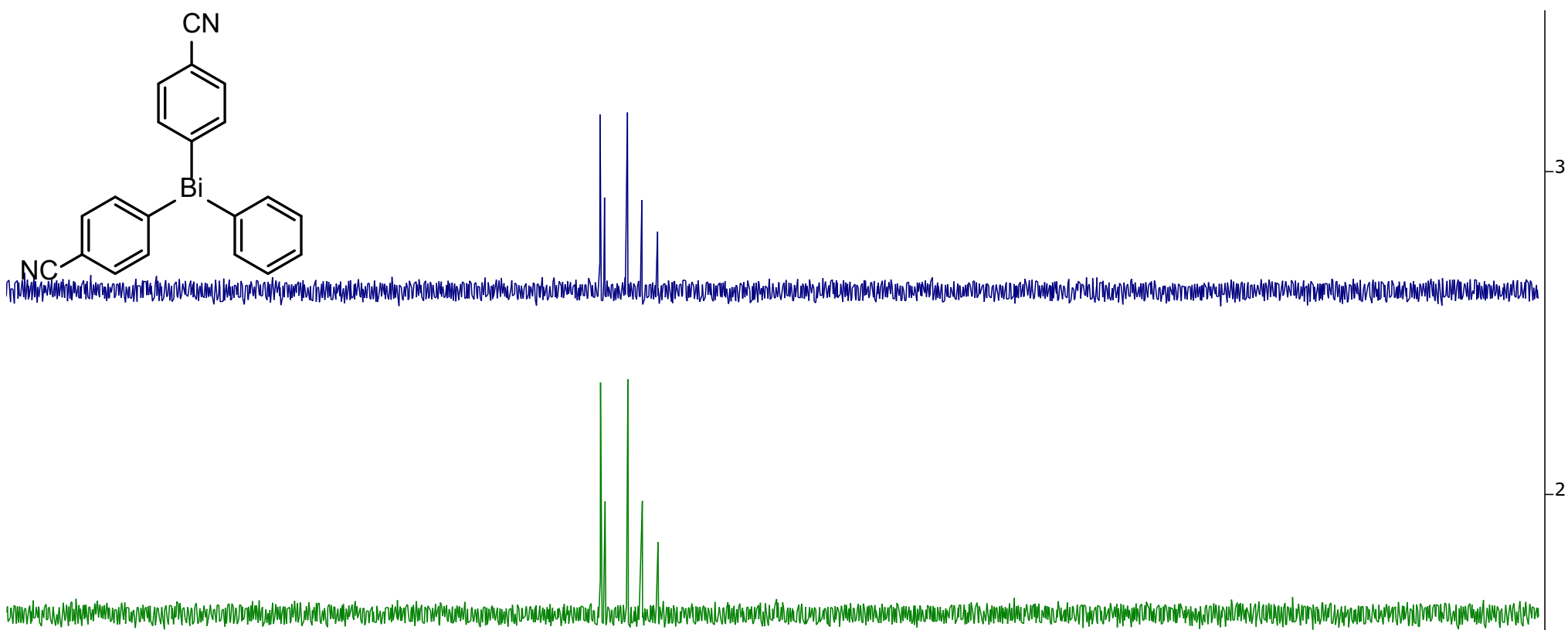

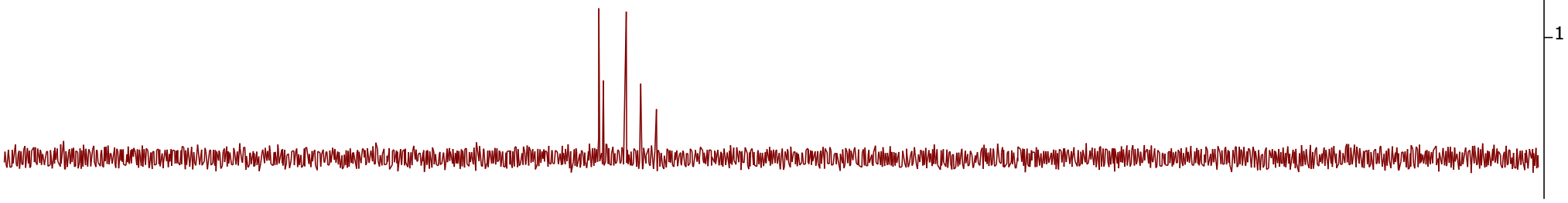

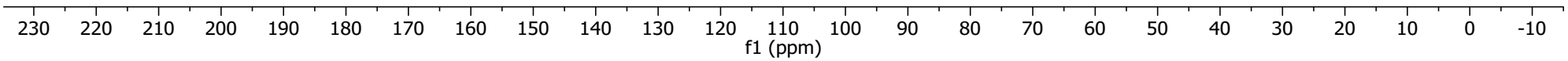

Figure S58. ${ }^{13} \mathrm{C}$ DEPT of $1 \mathrm{i}$ in $\mathrm{CDCl}_{3}$ 


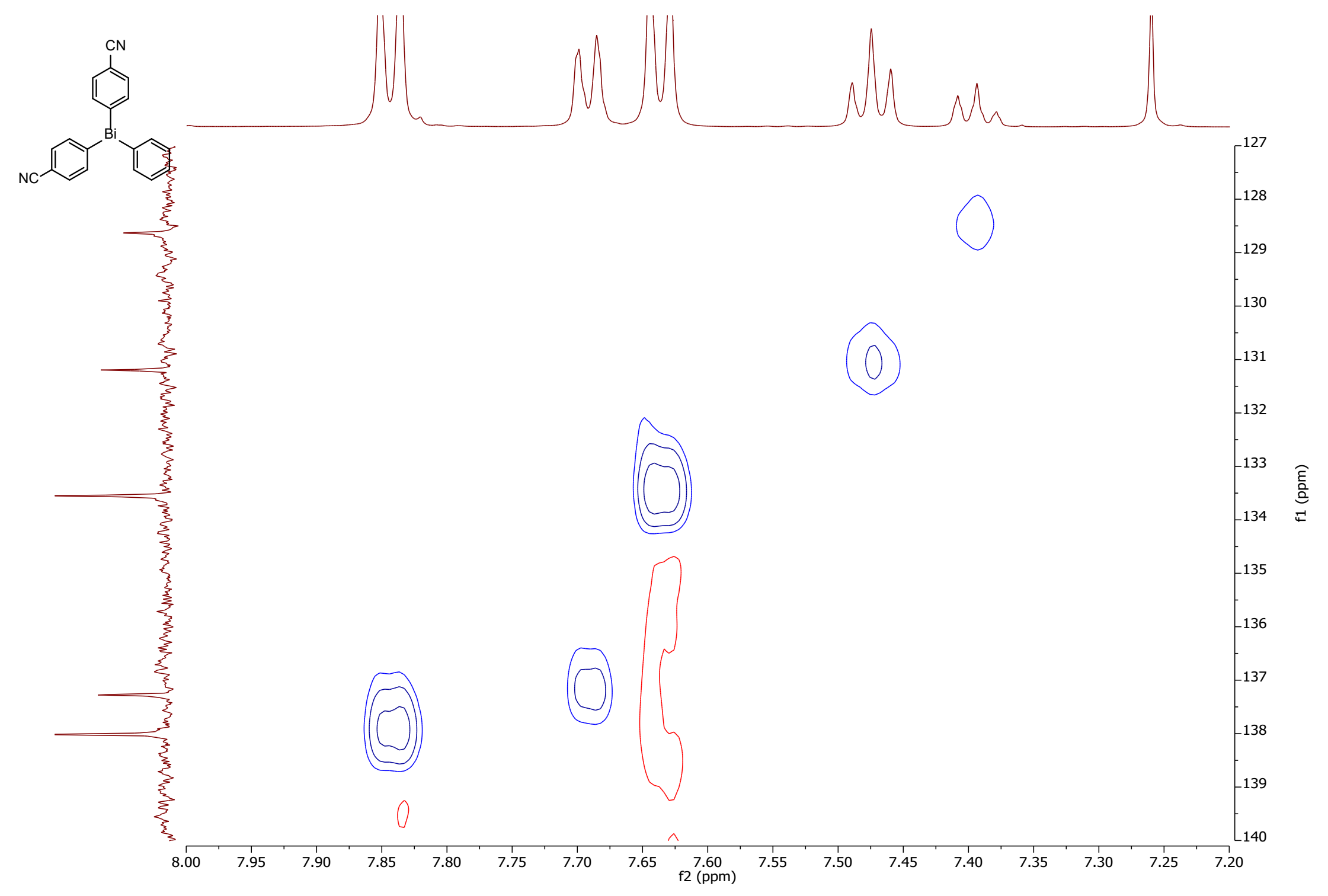

Figure S59. 2D HSQC of $1 \mathrm{i}$ in $\mathrm{CDCl}_{3}$ 


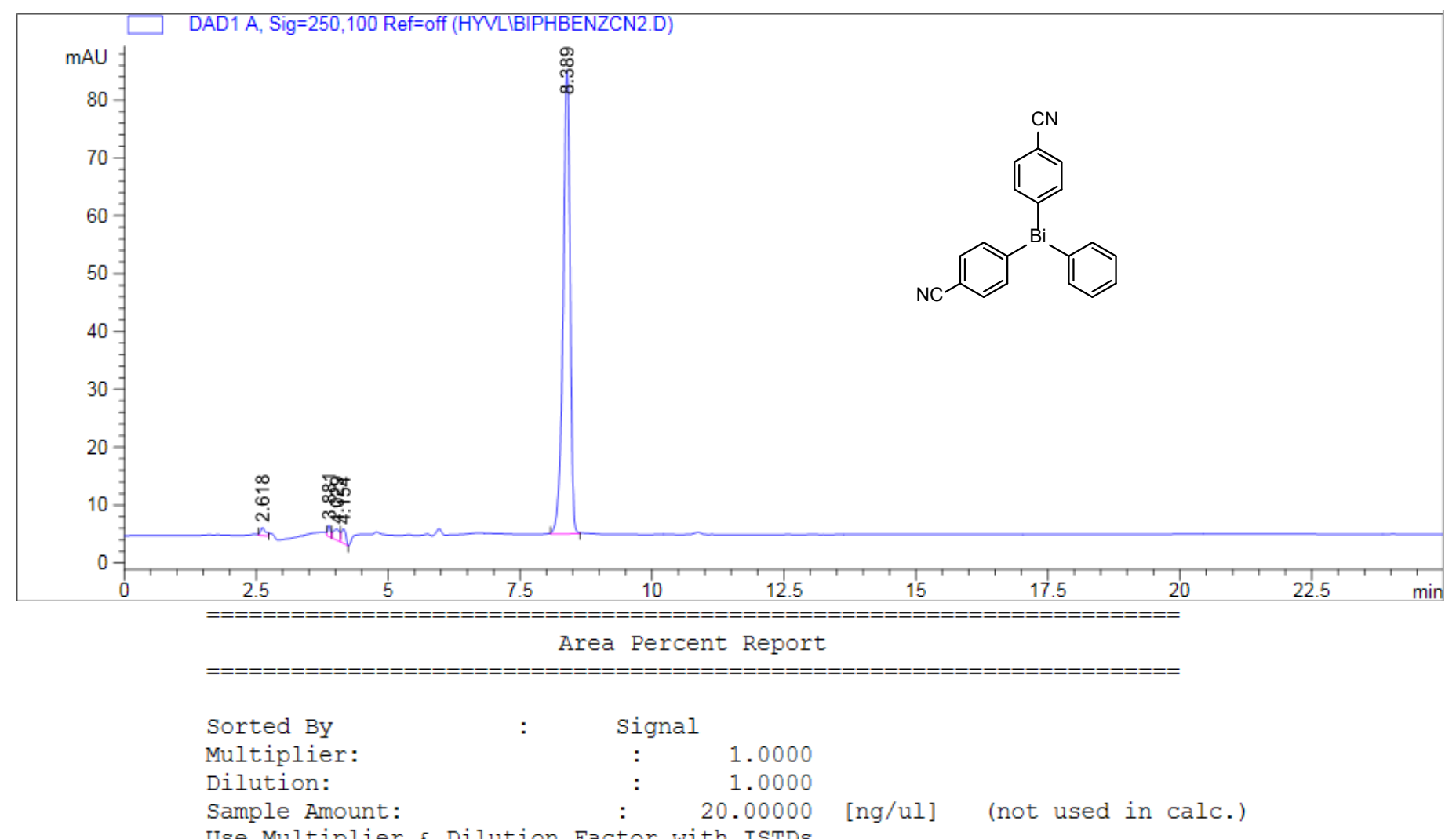

Use Multiplie

Signal 1: DAD1 A, Sig=250,100 Ref=off

\begin{tabular}{|c|c|c|c|c|c|c|}
\hline $\begin{array}{c}\text { Peak } \\
\quad \#\end{array}$ & $\begin{array}{l}\text { RetTime } \\
\text { [min] }\end{array}$ & Type & $\begin{array}{c}\text { Width } \\
\text { [min] }\end{array}$ & $\begin{array}{c}\text { Area } \\
{\left[\mathrm{mAU}^{*} \mathrm{~S}\right]}\end{array}$ & $\begin{array}{l}\text { Height } \\
\text { [MAU] }\end{array}$ & $\begin{array}{c}\text { Area } \\
\frac{\circ}{0}\end{array}$ \\
\hline 1 & 2.618 & $\mathrm{BB}$ & 0.0923 & 9.03760 & 138157 & 1.1502 \\
\hline 2 & 3.881 & $\mathrm{BV}$ & 0.0581 & 7.41773 & 1.96129 & 0.9440 \\
\hline 3 & 4.029 & VV & 0.1099 & 15.81809 & 1.92457 & 2.0131 \\
\hline 4 & 4.154 & VV & 0.0928 & 14.38671 & 2.43910 & 1.8310 \\
\hline 5 & 8.389 & $\mathrm{BB}$ & 0.1415 & 739.08337 & 80.22154 & 94.0617 \\
\hline
\end{tabular}

Figure S60. HPLC Chromatogram of 1i 


\section{NMR Spectra and EA Report of Compound 1j}<smiles>COc1ccc([Bi](c2ccccc2)c2ccc(OC)cc2)cc1</smiles>

Chemical Formula: $\mathrm{BiO}_{2} \mathrm{C}_{20} \mathrm{H}_{19}$

Molecular Weight: 500.35

Elemental Analysis: C: 48.01; Bi: 41.77; H: 3.83; O: 6.40

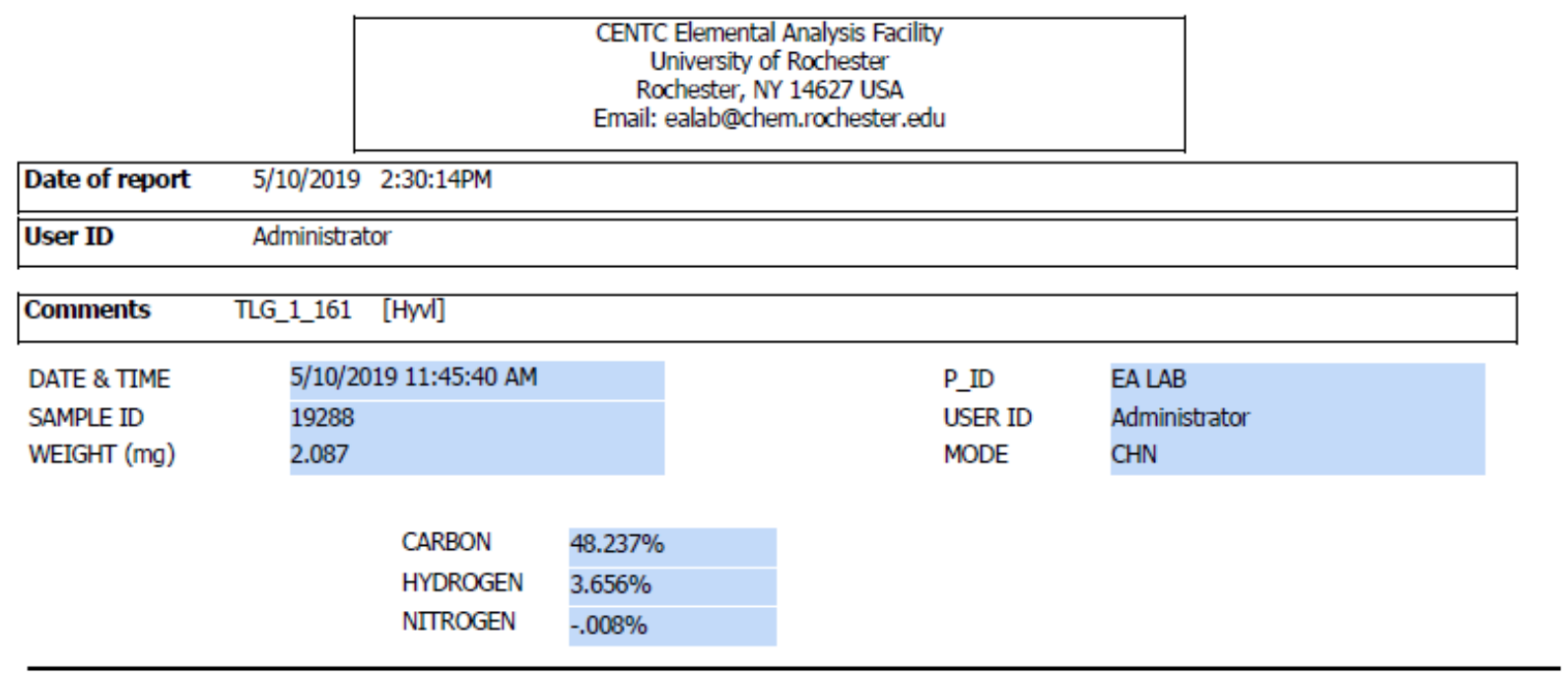

Acknowledgment

Analytical data were obtained from the CENTC Elemental Analysis Facility at the University of Rochester, funded by NSF CHE-0650456.

Instrumentation

Microanalysis samples were weighed with a PerkinElmer Model AD6000 Autobalance and their compositions were determined with a PerkinElmer 2400 Series II Analyzer.

Figure S61. EA report for $\mathbf{1 j}$ 


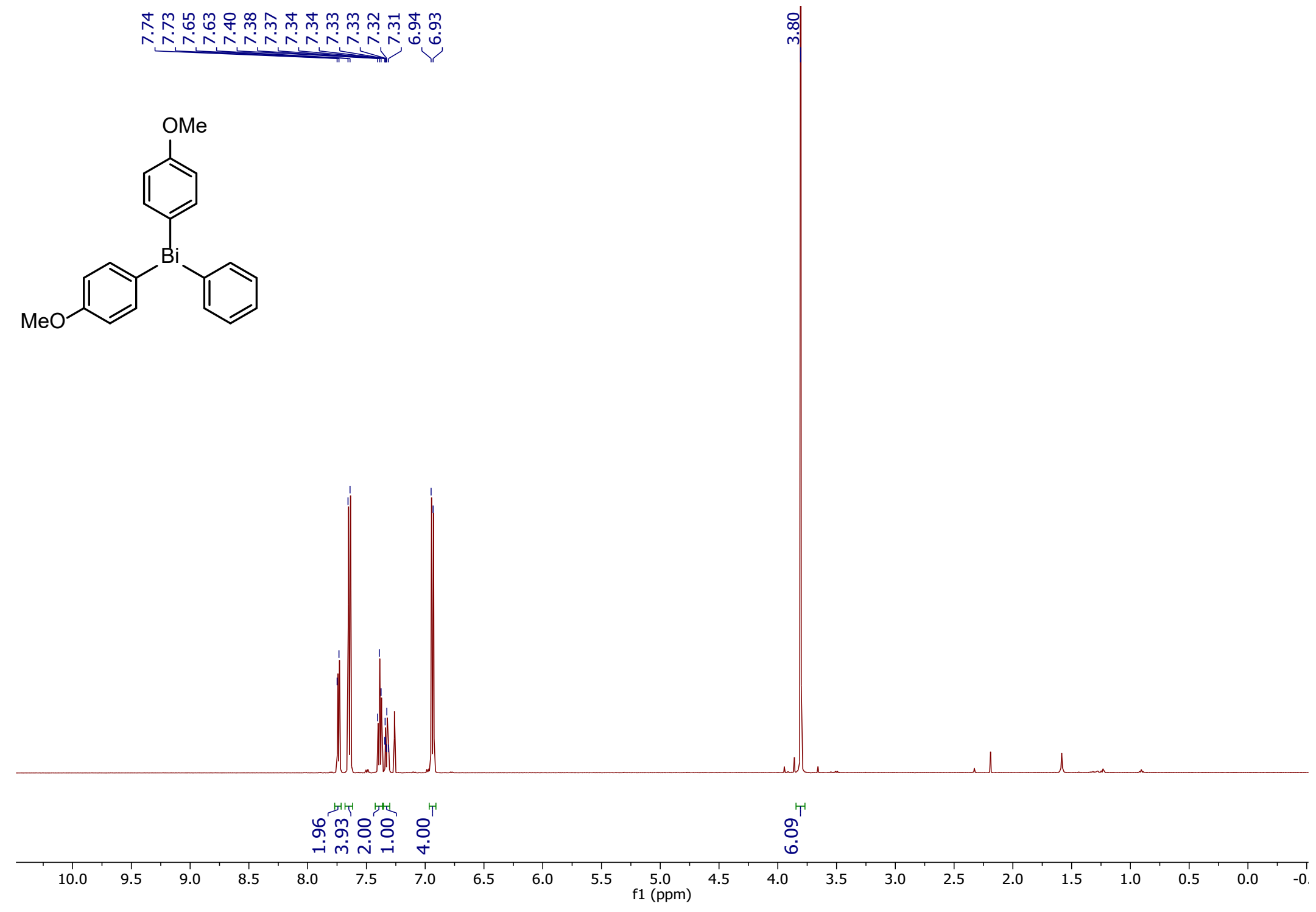

Figure S62. ${ }^{1} \mathrm{H}$ NMR of $\mathbf{1 j}$ in $\mathrm{CDCl}_{3}$ 


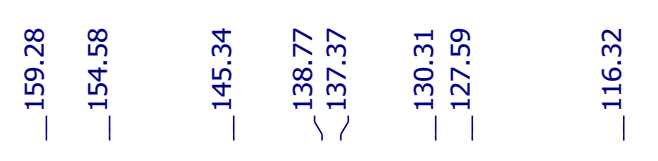

ํํำ
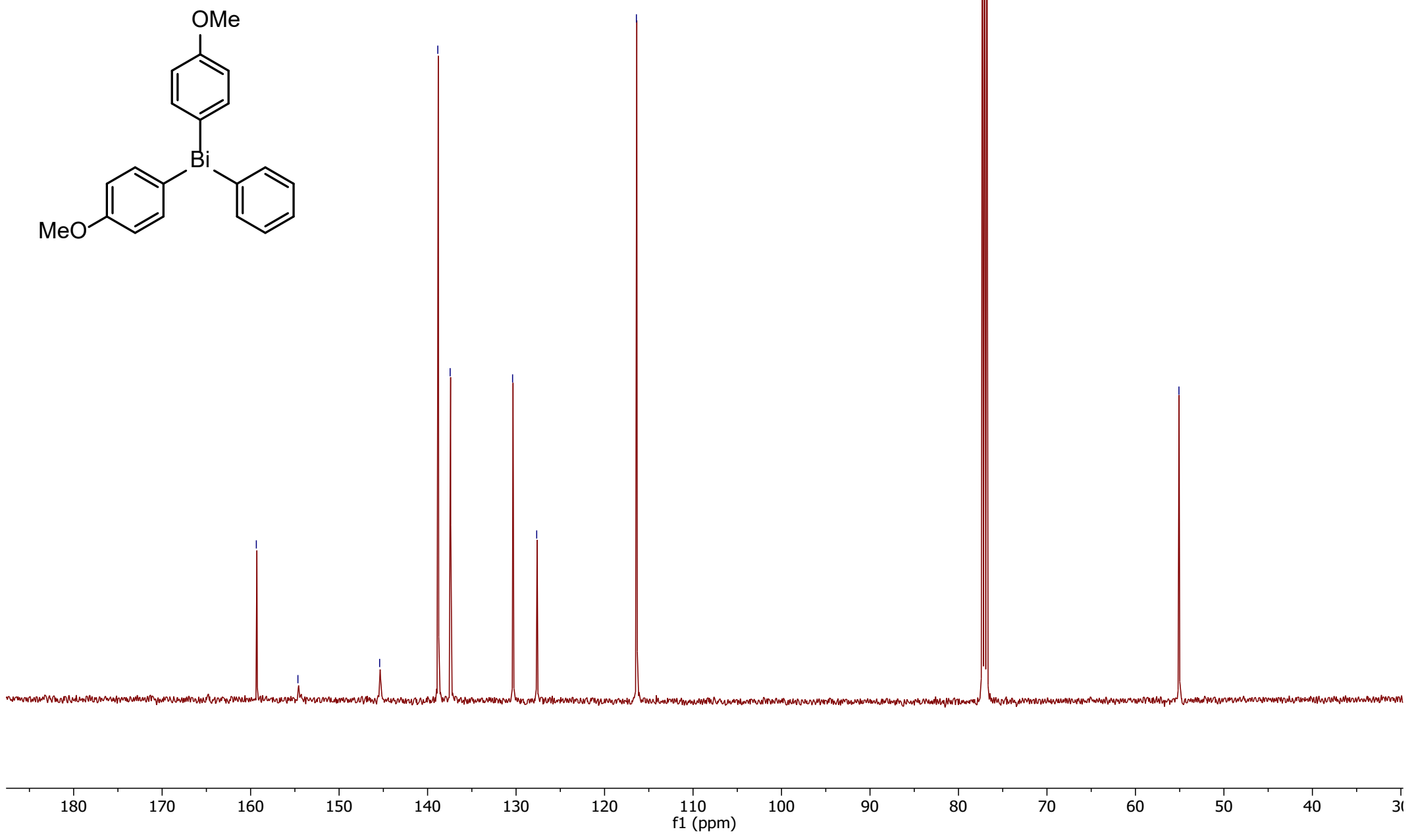

Figure S63. ${ }^{13} \mathrm{C}\left\{{ }^{1} \mathrm{H}\right\}$ NMR of $\mathbf{1 j}$ in $\mathrm{CDCl}_{3}$

S75 

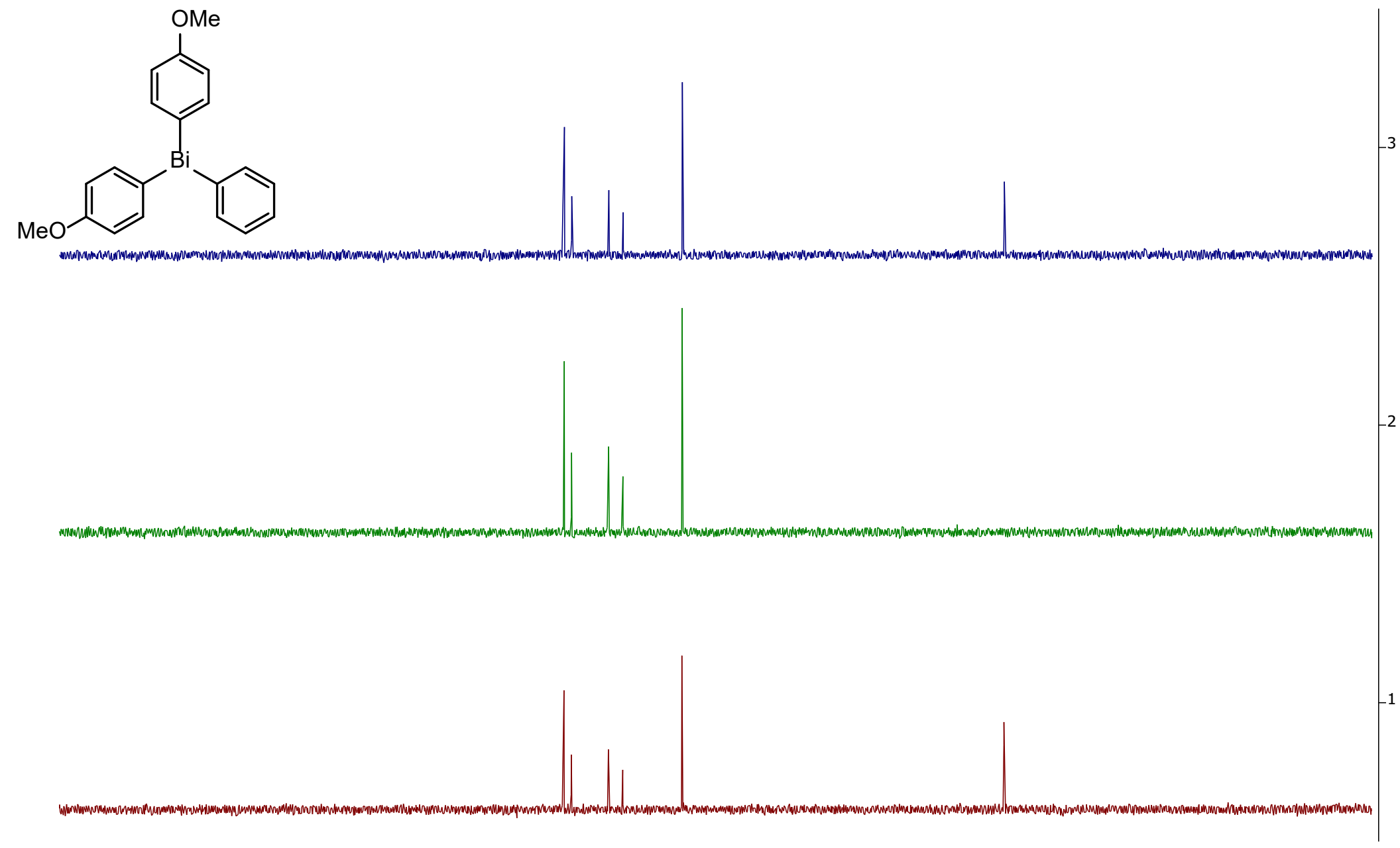

\begin{tabular}{lllllllllllllllllllllllll}
\hline 230 & 220 & 210 & 200 & 190 & 180 & 170 & 160 & 150 & 140 & 130 & 120 & 110 & 100 & 90 & 80 & 70 & 60 & 50 & 40 & 30 & 20 & 10 & 0 & -10
\end{tabular}

Figure S64. ${ }^{13} \mathrm{C}$ DEPT of $\mathbf{1 j}$ in $\mathrm{CDCl}_{3}$ 


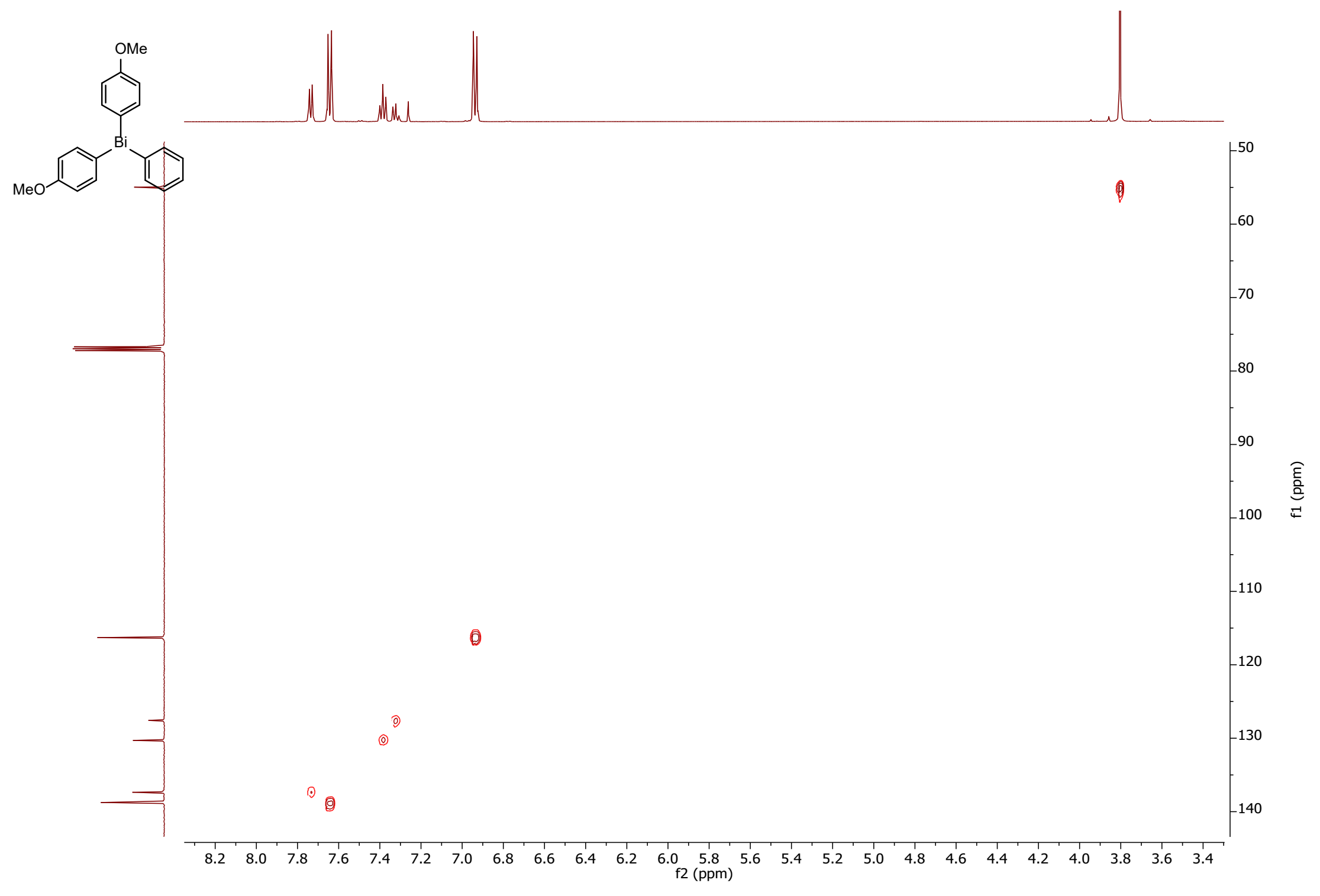

Figure S65. 2D HSQC of $\mathbf{1 j}$ in $\mathrm{CDCl}_{3}$ 


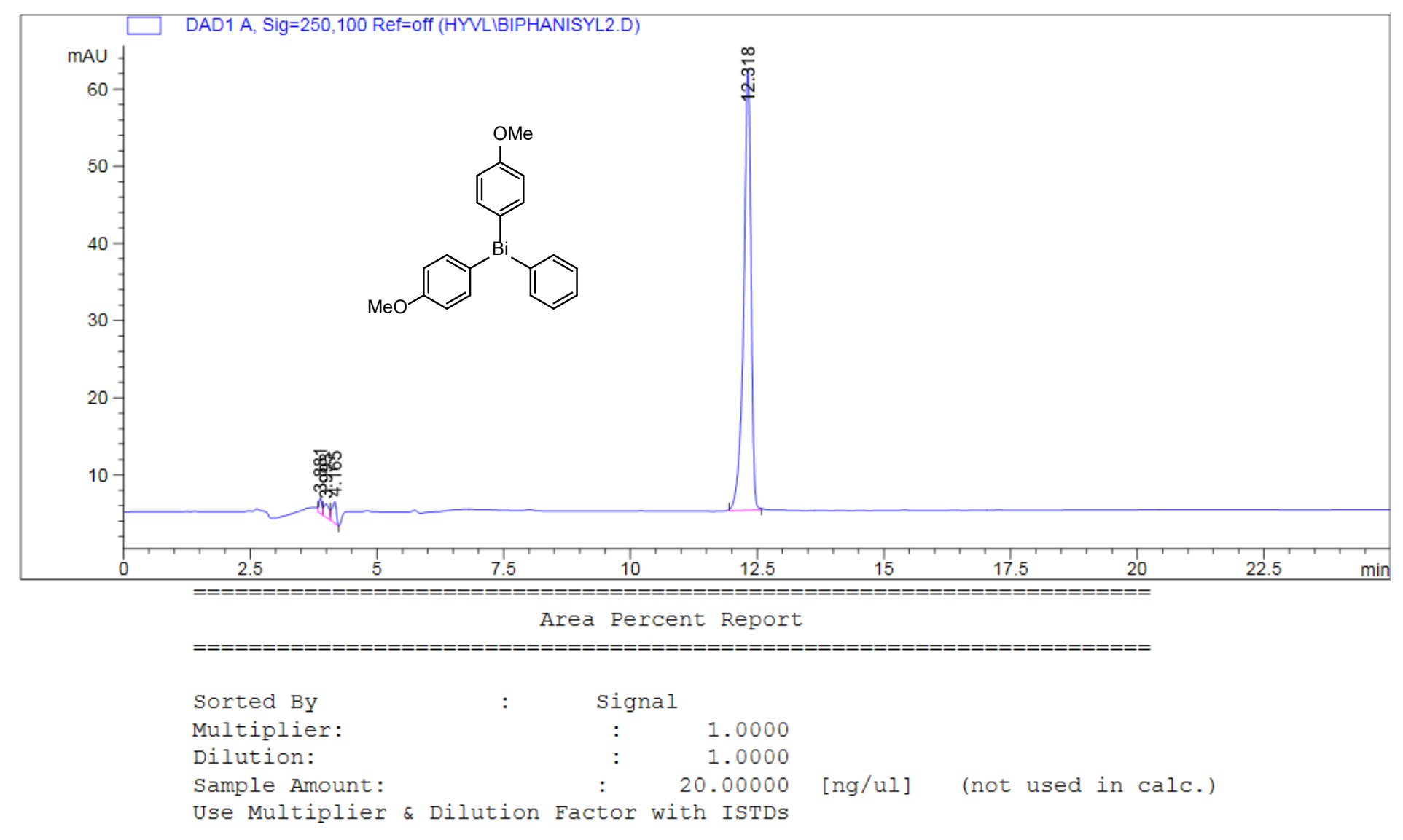

Signal 1: DAD1 A, Sig=250,100 Ref=off

\begin{tabular}{|c|c|c|c|c|c|c|}
\hline $\begin{array}{c}\text { Peak } \\
\#\end{array}$ & $\begin{array}{c}\text { RetTime } \\
\text { [min] }\end{array}$ & Type & $\begin{array}{c}\text { Width } \\
\text { [min] }\end{array}$ & $\begin{array}{c}\text { Area } \\
{\left[\mathrm{mAU}^{\star} \mathrm{s}\right]}\end{array}$ & $\begin{array}{l}\text { Height } \\
\text { [mAU] }\end{array}$ & $\begin{array}{c}\text { Area } \\
\&\end{array}$ \\
\hline-- & & & & --- & & -1 \\
\hline 1 & 3.881 & BV & 0.0568 & 7.76358 & 2.02089 & 1.2641 \\
\hline 2 & 3.993 & VV & 0.1062 & 13.08445 & 1.73126 & 2.1304 \\
\hline 3 & 4.165 & VV & 0.0849 & 15.91393 & 2.77373 & 2.5911 \\
\hline 4 & 12.318 & $\mathrm{BB}$ & 0.1516 & 577.41180 & 57.26262 & 94.0144 \\
\hline
\end{tabular}

Figure S66. HPLC Chromatogram of $\mathbf{1 j}$ 


\section{NMR Spectra and EA Report of Compound 1k}<smiles>Cc1ccc([Bi](c2ccc(C#N)cc2)c2ccc(C#N)cc2)cc1</smiles>

Chemical Formula: $\mathrm{BiN}_{2} \mathrm{C}_{21} \mathrm{H}_{15}$

Molecular Weight: 504.35

Elemental Analysis: C: 50.01; Bi: 41.44; H: 3.00; N: 5.55

CENTC Elemental Analysis Facility

University of Rochester

Rochester, NY 14627 USA

Email: ealab@chem.rochester.edu

\begin{tabular}{|c|c|c|c|c|}
\hline Date of report & $10 / 25 / 2019 \quad 6: 17: 32 \mathrm{PM}$ & & & \\
\hline User ID & Administrator & & & \\
\hline Comments & TLG_2_114B [Hyvl] & & & \\
\hline DATE \& TIME & 10/25/2019 3:06:16 PM & & P_ID & EA LAB \\
\hline SAMPLE ID & 19598 & & USER ID & Administrator \\
\hline WEIGHT (mg) & 2.160 & & MODE & $\mathrm{CHN}$ \\
\hline & CARBON & $50.008 \%$ & & \\
\hline & HYDROGEN & $2.868 \%$ & & \\
\hline & NITROGEN & $5.464 \%$ & & \\
\hline
\end{tabular}

Acknowledgment

Analytical data were obtained from the CENTC Elemental Analysis Facility at the University of Rochester, funded by NSF CHE-0650456.

Figure S67. EA report for $1 \mathrm{k}$ 


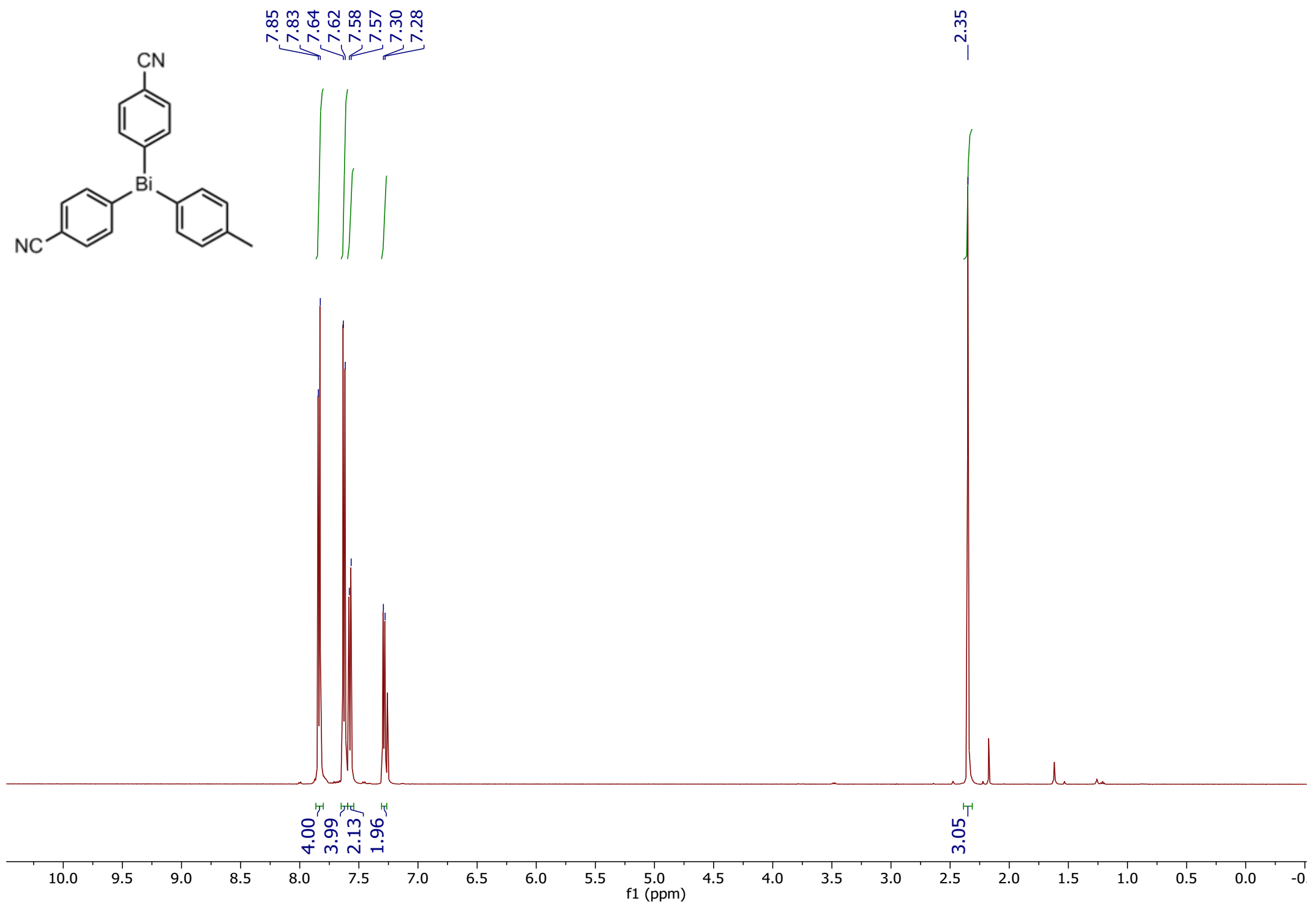

Figure S68. ${ }^{1} \mathrm{H}$ NMR of $\mathbf{1 k}$ in $\mathrm{CDCl}_{3}$

S80 
<smiles>Cc1ccc([Bi](c2ccc(C#N)cc2)c2ccc(C#N)cc2)cc1</smiles>

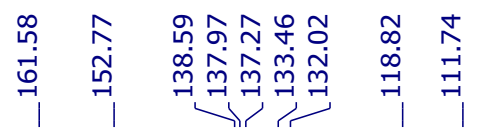

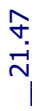

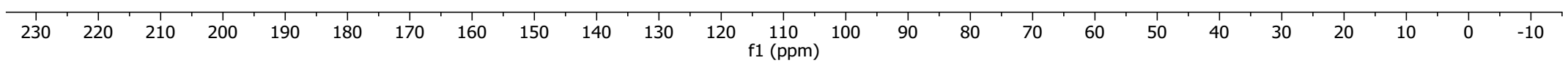

Figure S69. ${ }^{13} \mathrm{C}\left\{{ }^{1} \mathrm{H}\right\}$ NMR of $\mathbf{1 k}$ in $\mathrm{CDCl}_{3}$

S81 
<smiles>Cc1ccc([Bi](c2ccc(C#N)cc2)c2ccc(C#N)cc2)cc1</smiles>

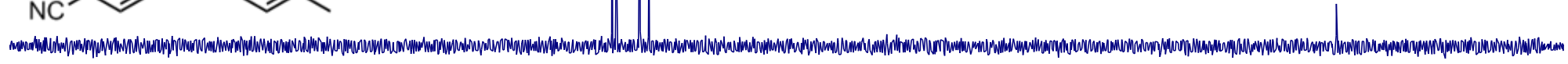
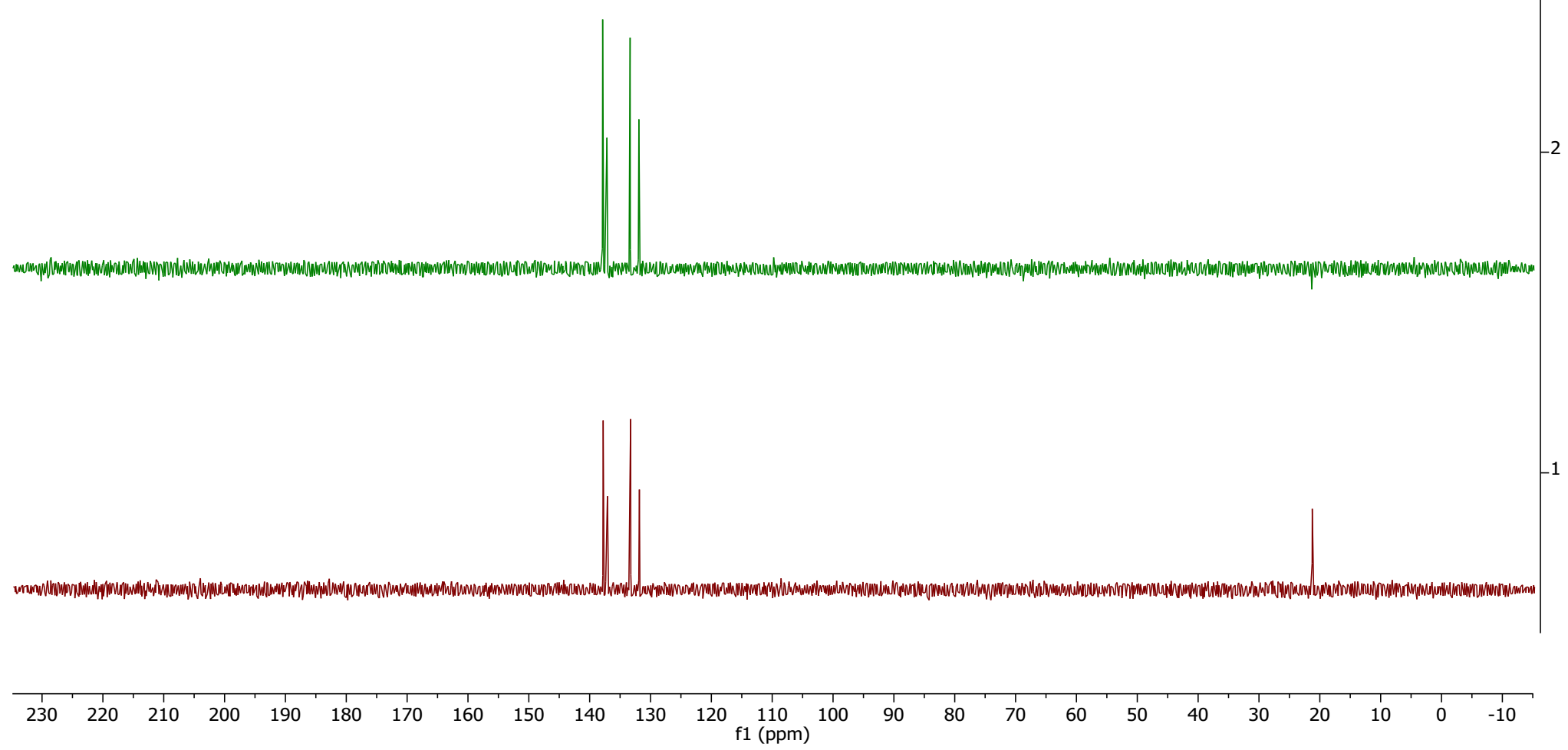

Figure $\mathbf{S 7 0} .{ }^{13} \mathrm{C}$ DEPT of $\mathbf{1 k}$ in $\mathrm{CDCl}_{3}$

S82 


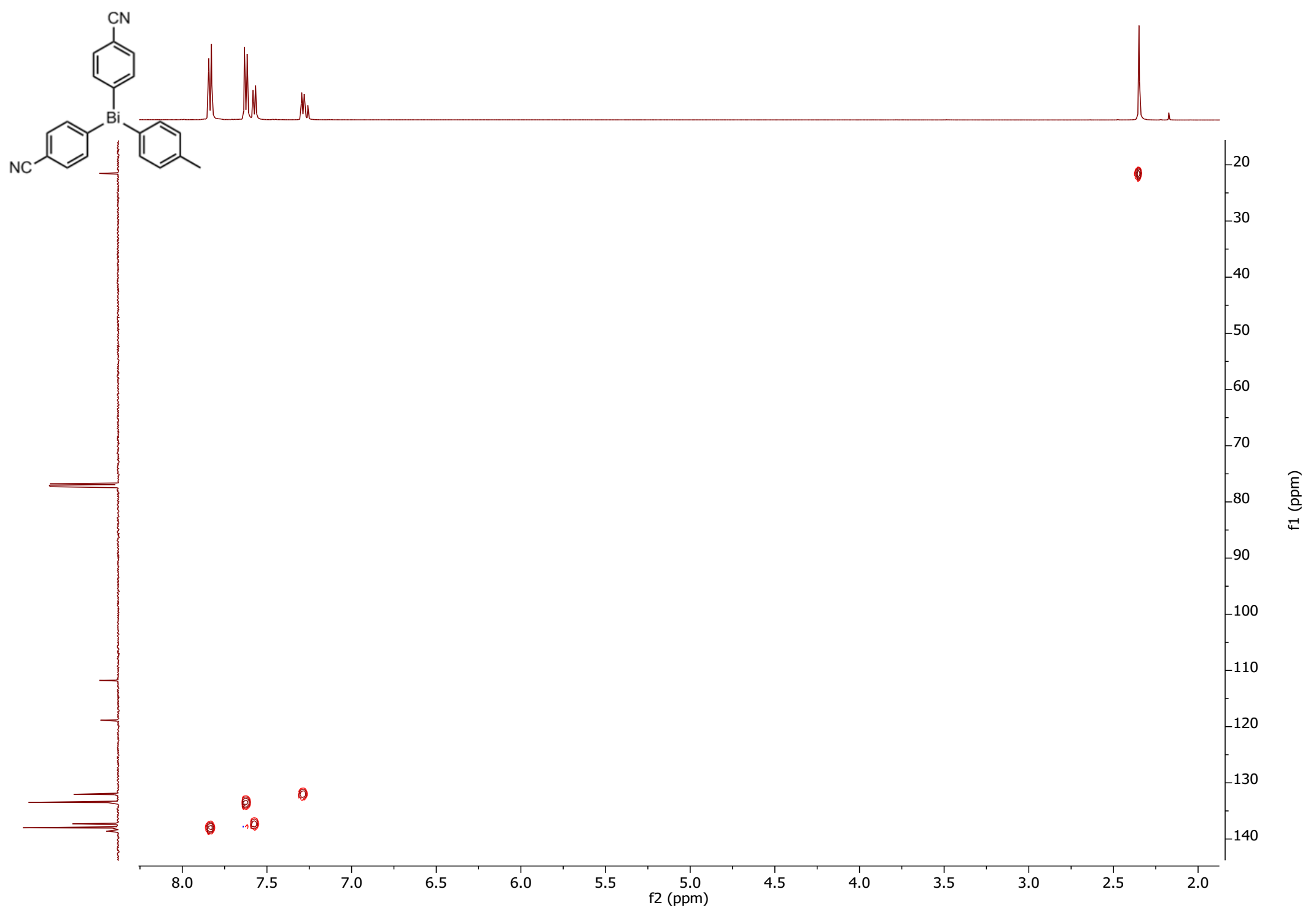

Figure S71. 2D HSQC of $1 \mathbf{k}$ in $\mathrm{CDCl}_{3}$ 


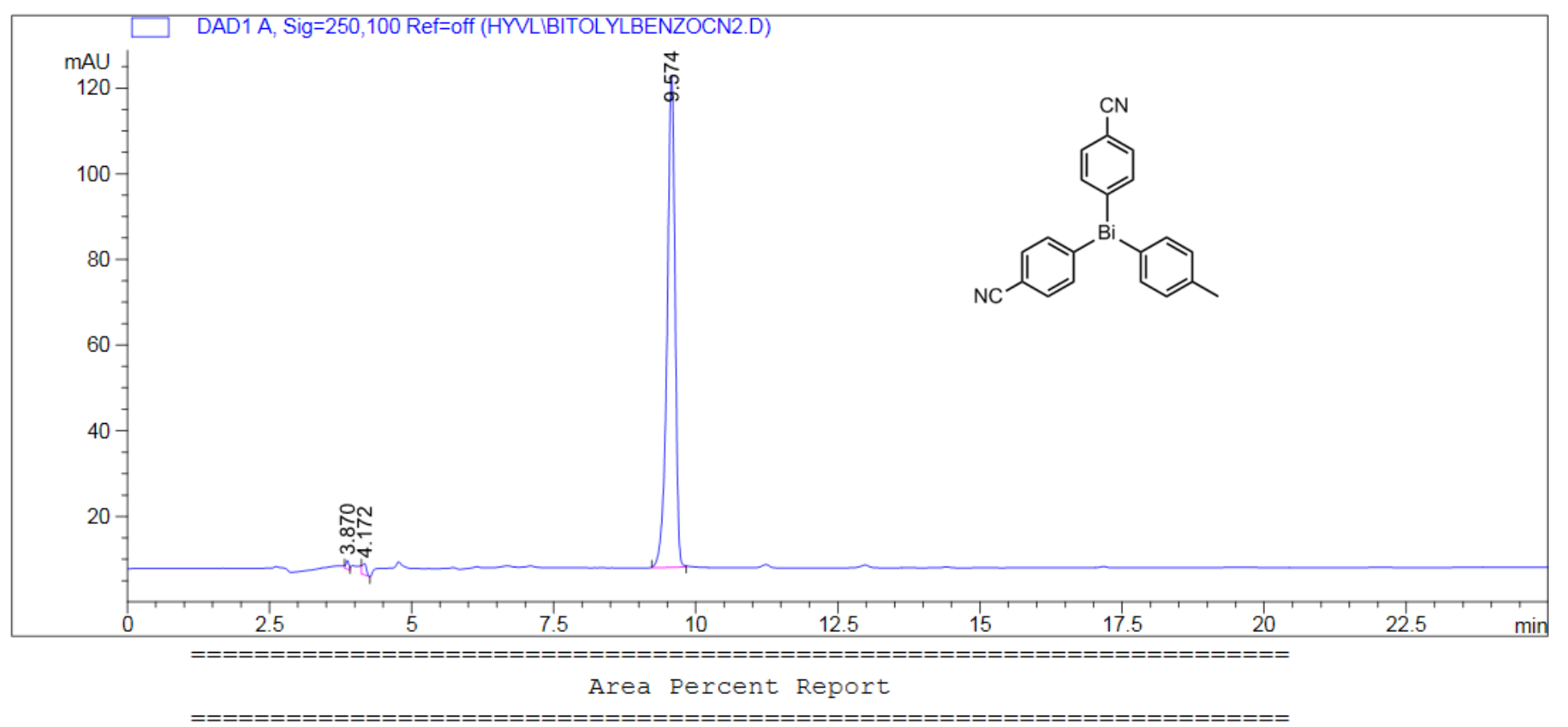

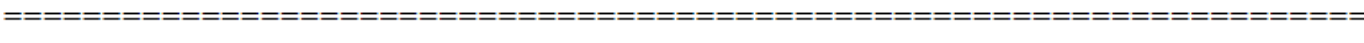

$\begin{array}{lcccc}\text { Sorted By } & : & \text { Signal } & \\ \text { Multiplier: } & : & 1.0000 & \\ \text { Dilution: } & : & 1.0000 & \\ \text { Sample Amount: } & : & 20.00000 & {[\mathrm{ng} / \mathrm{ul}]} & \text { (not used in calc.) }\end{array}$

Signal 1: DAD1 A, Sig=250,100 Ref=off

\begin{tabular}{|c|c|c|c|c|c|c|}
\hline $\begin{array}{c}\text { Peak } \\
\#\end{array}$ & $\begin{array}{c}\text { RetTime } \\
\text { [min] }\end{array}$ & Type & $\begin{array}{c}\text { Width } \\
\text { [min] }\end{array}$ & $\begin{array}{c}\text { Area } \\
{\left[\mathrm{mAU}^{*} \mathrm{~s}\right]}\end{array}$ & $\begin{array}{l}\text { Height } \\
\text { [mAU] }\end{array}$ & $\begin{array}{c}\text { Area } \\
\&\end{array}$ \\
\hline- & 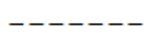 & & -- & ---- & ----0 & -----1 \\
\hline 1 & 3.870 & BV & 0.0561 & 7.54989 & 1.99554 & 0.6639 \\
\hline 2 & 4.172 & BV & 0.0763 & 13.27818 & 2.65567 & 1.1676 \\
\hline 3 & 9.574 & $\mathrm{BB}$ & 0.1493 & 1116.35815 & 114.92860 & 98.1685 \\
\hline
\end{tabular}

Figure S72. HPLC Chromatogram of $\mathbf{1 k}$ 


\section{NMR Spectra and EA Report of Compound 11}<smiles>COc1ccc(B(c2ccc(C)cc2)c2ccc(OC)cc2)cc1</smiles>

Chemical Formula: $\mathrm{BiO}_{2} \mathrm{C}_{21} \mathrm{H}_{21}$

Molecular Weight: 514.38

Elemental Analysis: C: 49.04; Bi: 40.63; H: 4.12; O: 6.22

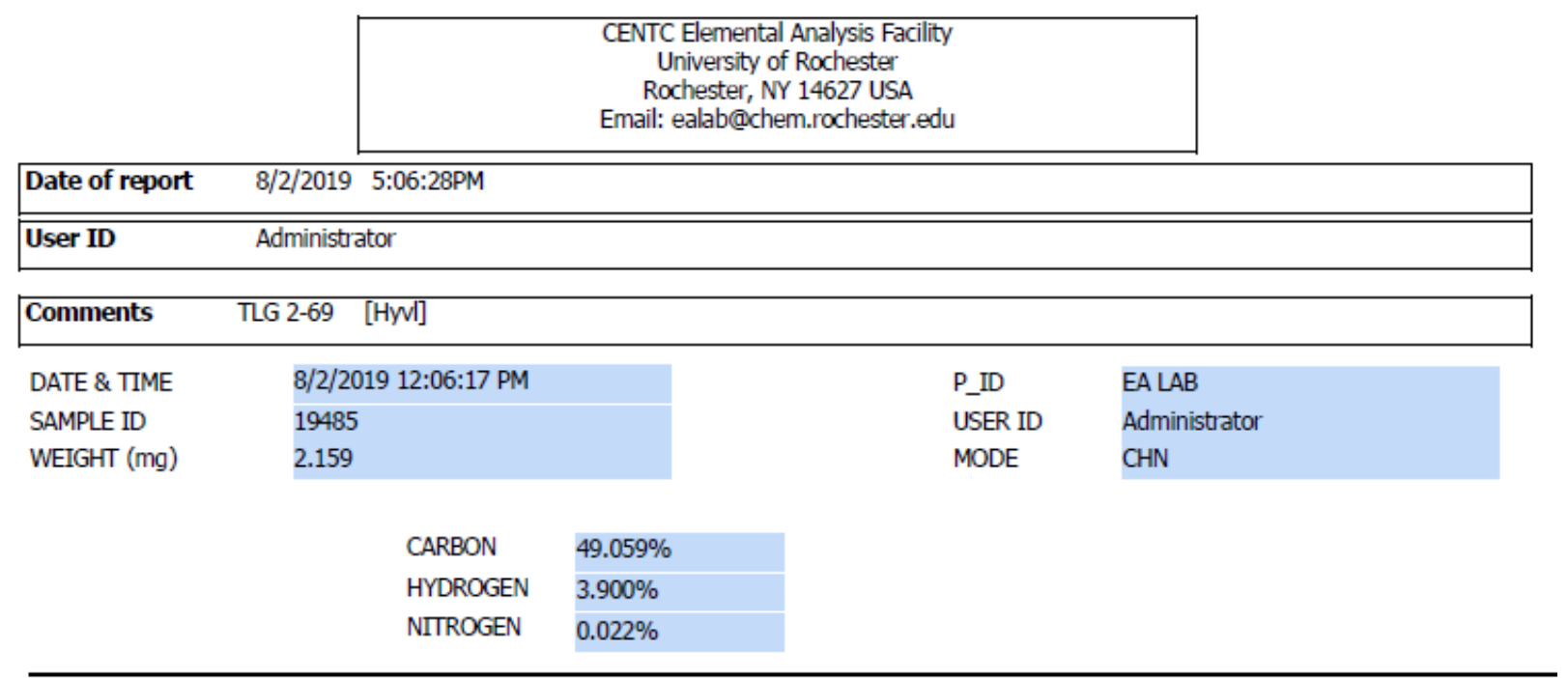

\section{Acknowledgment}

Analytical data were obtained from the CENTC Elemental Analysis Facility at the University of Rochester, funded by NSF CHE-0650456.

\section{Instrumentation}

Microanalysis samples were weighed with a PerkinElmer Model AD6000 Autobalance and their compositions were determined with a PerkinElmer 2400 Series II Analyzer.

Figure S73. EA report for 11 

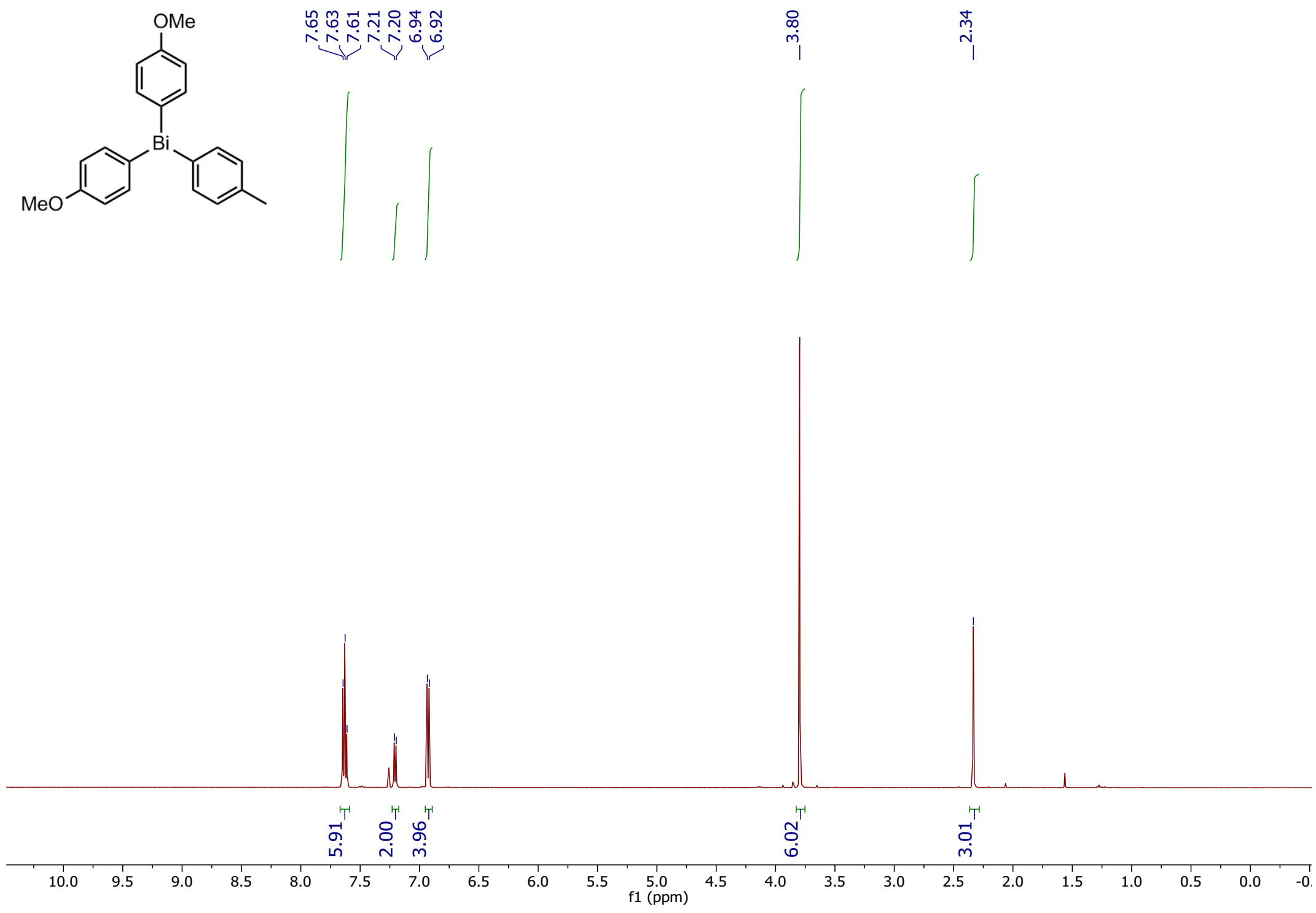

Figure S74. ${ }^{1} \mathrm{H}$ NMR of 11 in $\mathrm{CDCl}_{3}$ 


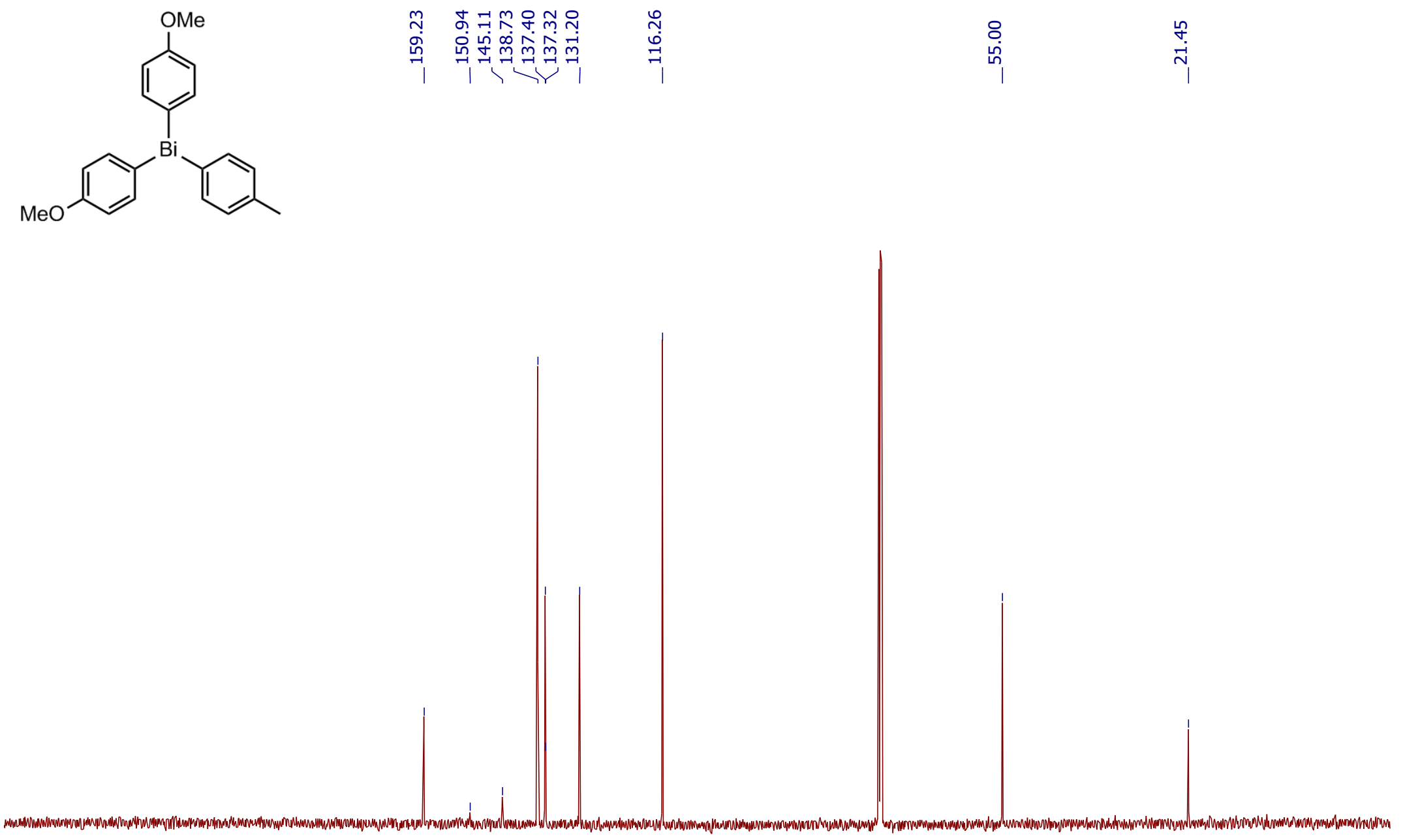

\begin{tabular}{llllllllllllllllllllllllll}
\hline & 230 & 220 & 210 & 200 & 190 & 180 & 170 & 160 & 150 & 140 & 130 & 120 & 110 & 100 & 90 & 80 & 70 & 60 & 50 & 40 & 30 & 20 & 10 & 0 & -10
\end{tabular}

Figure S75. ${ }^{13} \mathrm{C}\left\{{ }^{1} \mathrm{H}\right\}$ NMR of $\mathbf{1 l}$ in $\mathrm{CDCl}_{3}$ 

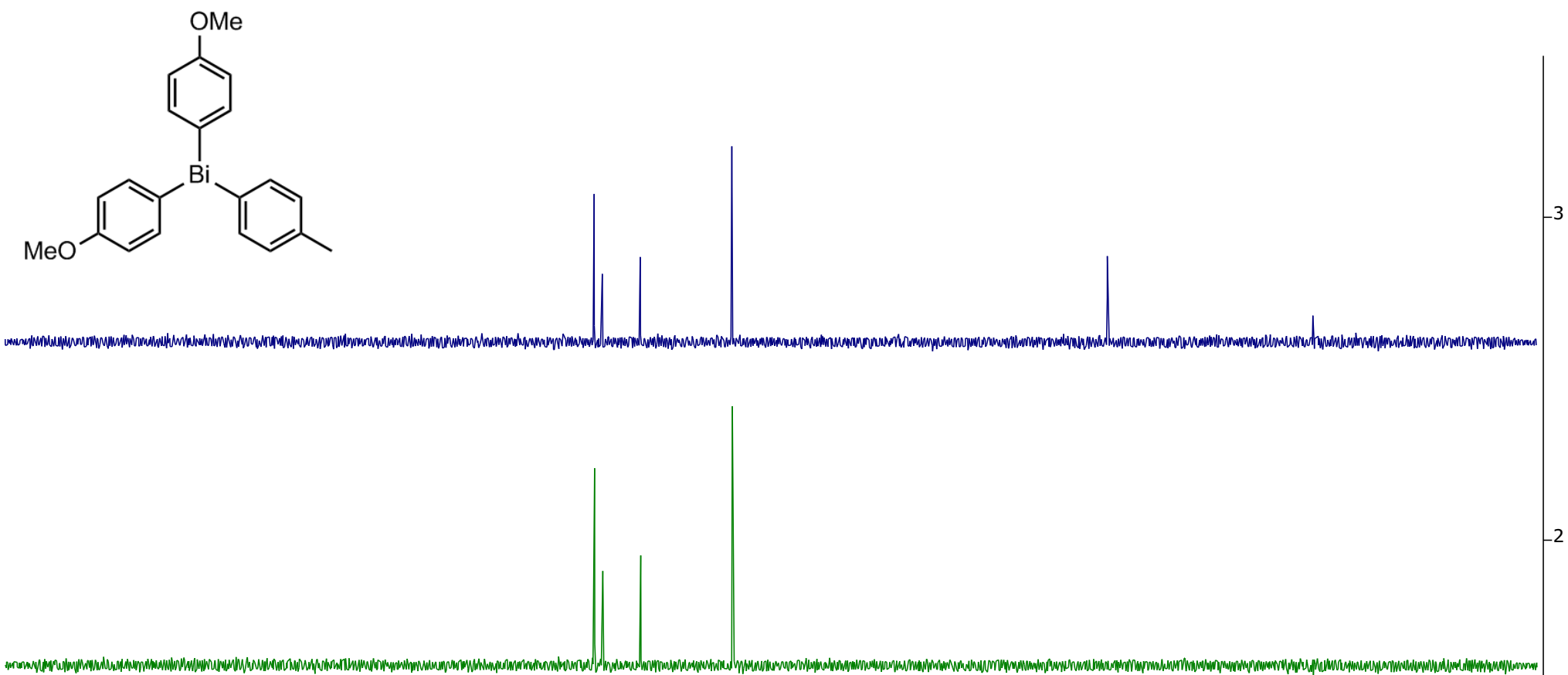

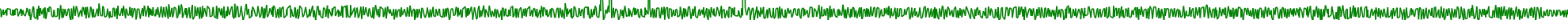

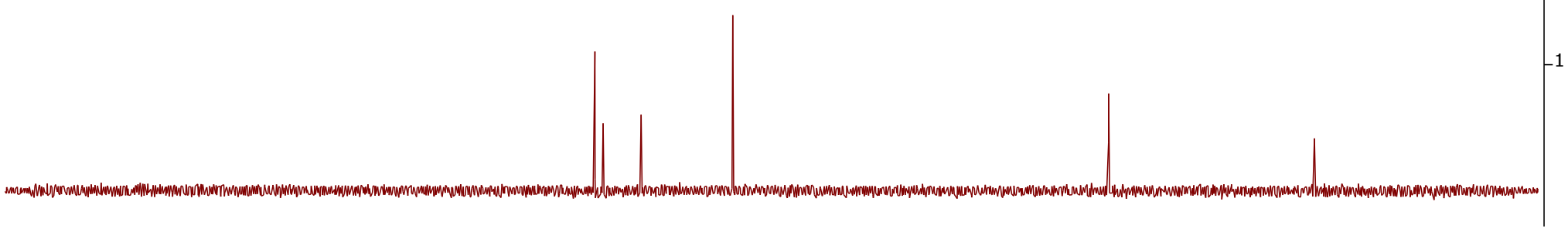

\begin{tabular}{lllllllllllllllllllllllllll}
\hline 230 & 220 & 210 & 200 & 190 & 180 & 170 & 160 & 150 & 140 & 130 & 120 & 110 & 100 & 90 & 80 & 70 & 60 & 50 & 40 & 30 & 20 & 10 & 0 & -10
\end{tabular}

Figure S76. ${ }^{13} \mathrm{C}$ DEPT of 11 in $\mathrm{CDCl}_{3}$ 


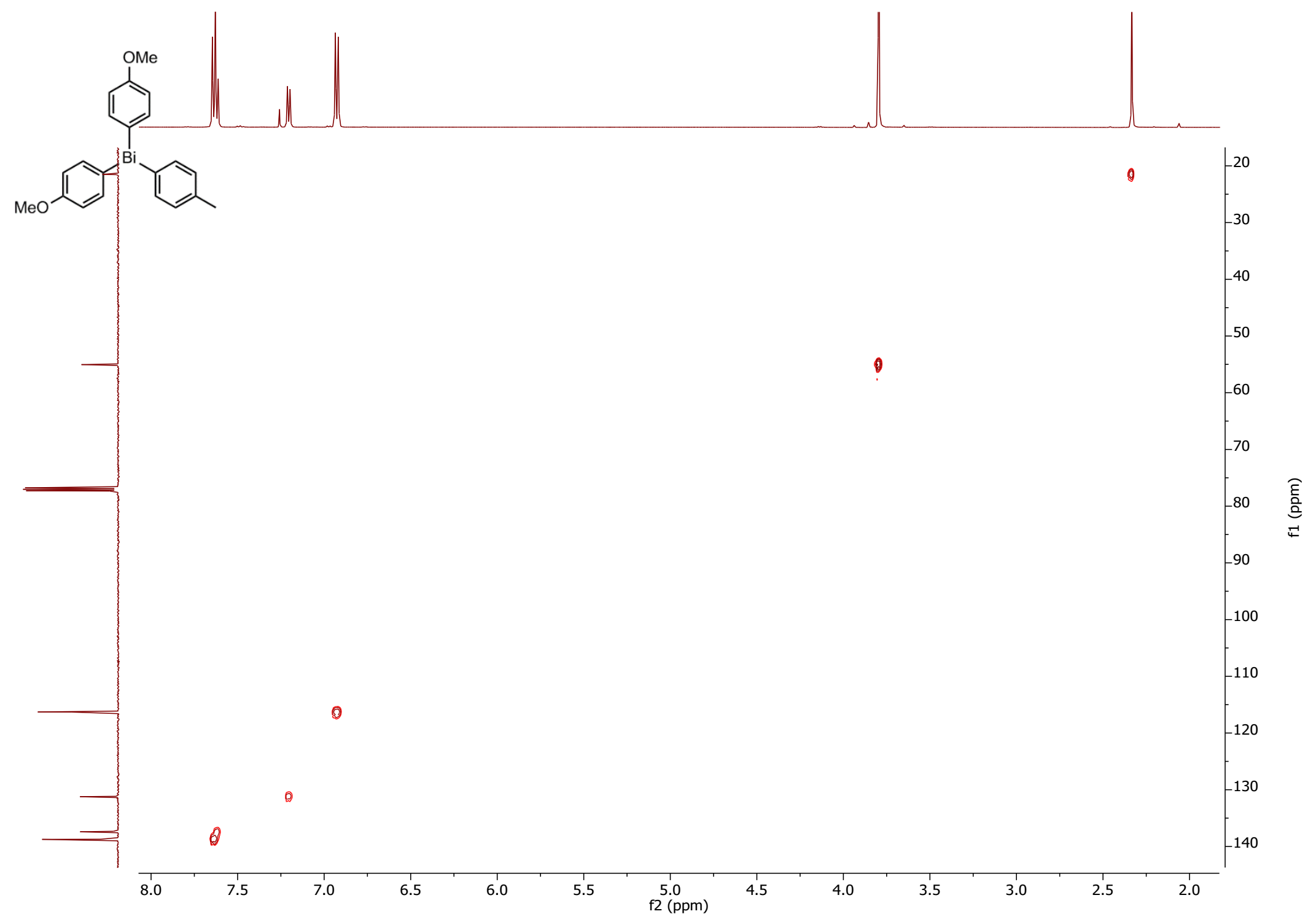

Figure S77. 2D HSQC of 11 in $\mathrm{CDCl}_{3}$ 


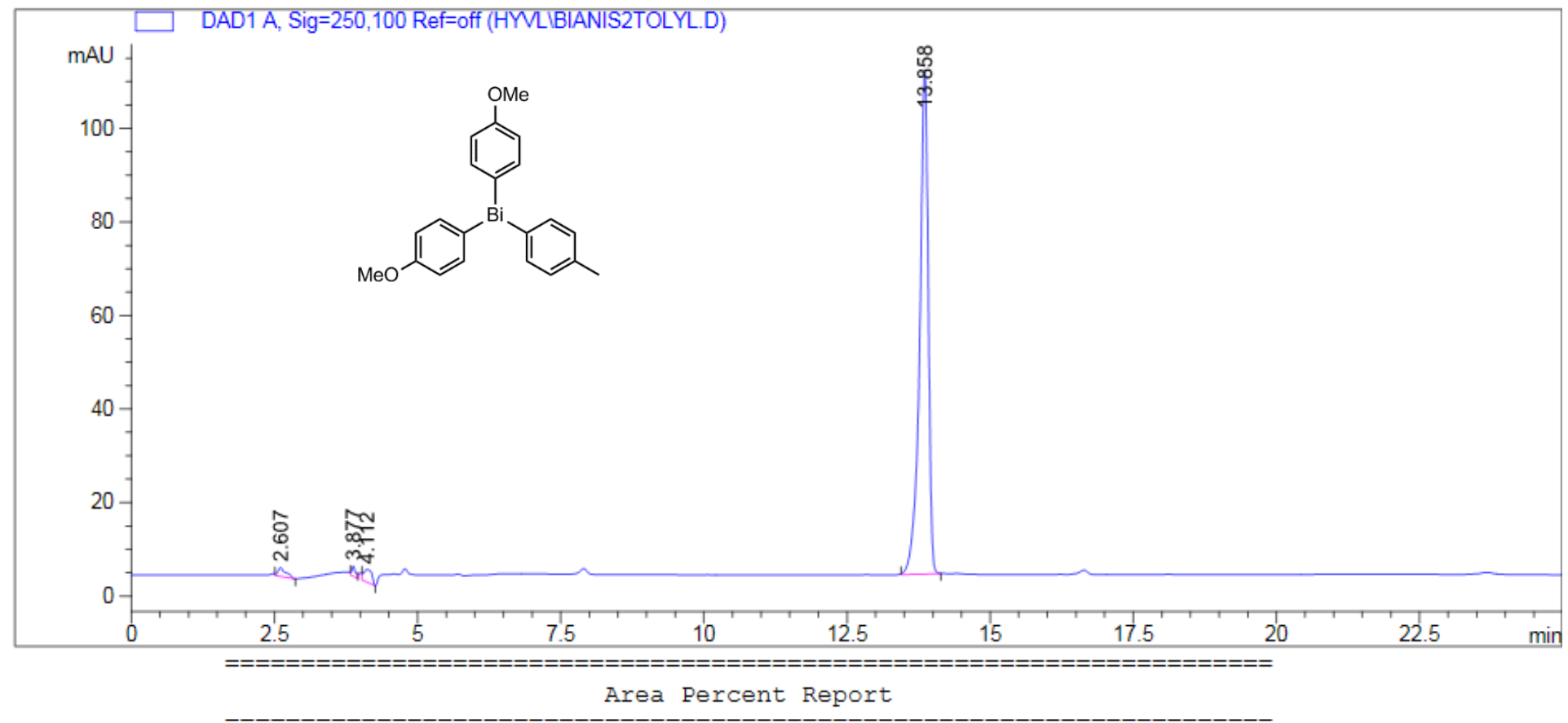

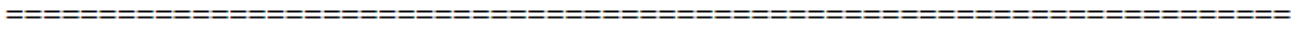

\begin{tabular}{lcrrr} 
Sorted By & $:$ & \multicolumn{2}{c}{ Signal } & \\
Multiplier: & $:$ & 1.0000 & \\
Dilution: & $:$ & 1.0000 & \\
Sample Amount: & $:$ & 20.00000 & [ng/ul] & (not used in calc.)
\end{tabular}

Use Multiplier \& Dilution Factor with ISTDs

Signal 1: DAD1 A, Sig=250,100 Ref=off

\begin{tabular}{|c|c|c|c|c|c|c|}
\hline \# & $\begin{array}{c}\text { RetTime } \\
\text { [min] }\end{array}$ & Type & $\begin{array}{c}\text { Width } \\
\text { [min] }\end{array}$ & $\begin{array}{c}\text { Area } \\
{\left[\mathrm{mAU} U^{\star} \mathrm{s}\right]}\end{array}$ & $\begin{array}{l}\text { Height } \\
\text { [mAU] }\end{array}$ & $\begin{array}{c}\text { Area } \\
\frac{8}{\delta}\end{array}$ \\
\hline & & & & & & \\
\hline 1 & 2 & $B$ & & 18. & & 62 \\
\hline 2 & 77 & BB & & 10 & & 28 \\
\hline 3 & 4.112 & BV & 0.1 & 26.66670 & 689 & 2.2556 \\
\hline 4 & 13.858 & $\mathrm{BB}$ & 0.1559 & 1127.08960 & 107.88406 & 95.3354 \\
\hline
\end{tabular}

Figure S78. HPLC Chromatogram of 11 
8. HPLC Chromatogram of 1:1:1:1 mixture of tri(4-methoxyphenyl)bismuthane, di(4-methoxyphenyl)phenylbismuthane (1j), diphenyl(4methoxyphenyl)bismuthane (1b), and triphenylbismuthane used for monitoring of dismutation process in Scheme 2

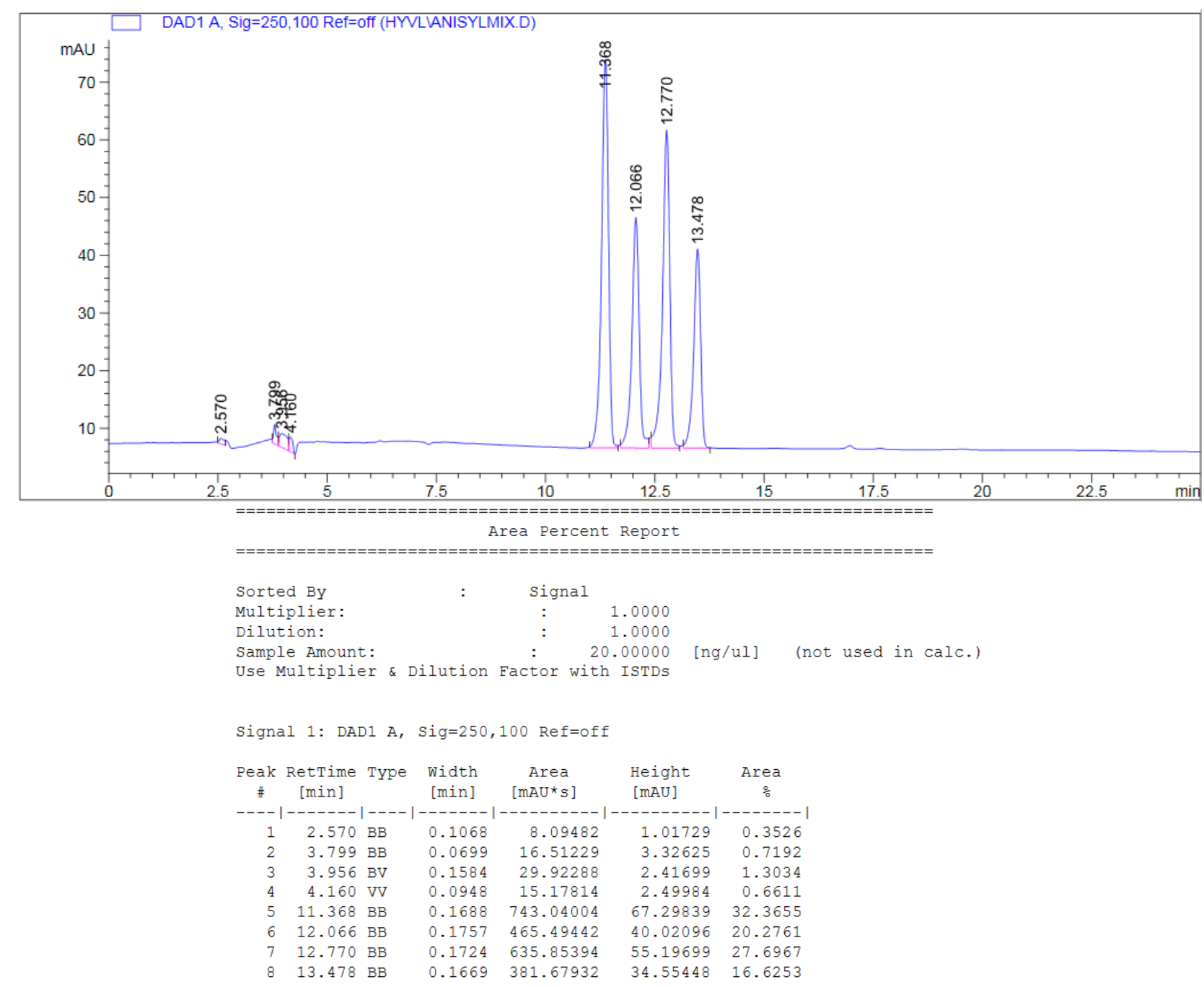

Figure S79. HPLC Chromatogram of 1:1:1:1 mixture of tri(4-methoxyphenyl)bismuthane, di(4-methoxyphenyl)phenylbismuthane (1j), diphenyl(4-methoxyphenyl)bismuthane (1b) and triphenylbismuthane 


\section{Crystal Structure Data for Compound Di(4-cyanophenyl)(p-tolyl)bismuthane $1 \mathrm{k}$}

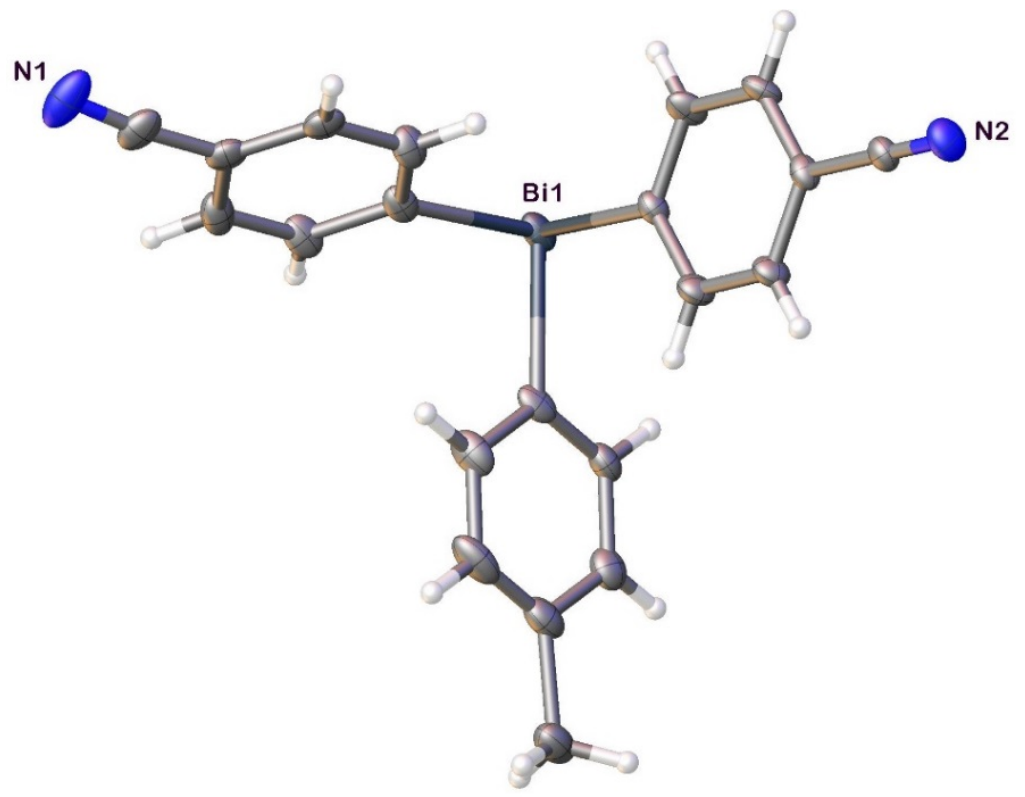

Figure S80. Solved Crystal Structure of Di(4-cyanophenyl)(p-tolyl)bismuthane 1k

Table S2. Crystal data and structure refinement for hyvl12 $0 \mathrm{~m}$ a.

Identification code

Empirical formula

Formula weight

Temperature

Wavelength

Crystal system

Space group

Unit cell dimensions

Volume

Z

Density (calculated)

Absorption coefficient

$\mathrm{F}(000)$

Crystal size

Theta range for data collection

Index ranges

Reflections collected

Independent reflections

Completeness to theta $=25.242^{\circ}$

Absorption correction

Max. and min. transmission

Refinement method

Data / restraints / parameters

Goodness-of-fit on $\mathrm{F}^{2}$

Final $R$ indices [I $>2 \operatorname{sigma}(\mathrm{I})]$

$\mathrm{R}$ indices (all data)

Extinction coefficient

Largest diff. peak and hole
TLG 2-46

C21 H15 Bi N2

504.33

$100.0 \mathrm{~K}$

$0.71073 \AA$

Triclinic

P-1

$\mathrm{a}=8.1718(10) \AA$

$\alpha=105.132(4)^{\circ}$.

$\mathrm{b}=9.9011(14) \AA$

$\beta=105.299(4)^{\circ}$.

$\mathrm{c}=12.3098(15) \AA$

$\gamma=90.697(4)^{\circ}$.

923.9(2) $\AA^{3}$

2

$1.813 \mathrm{Mg} / \mathrm{m}^{3}$

$9.545 \mathrm{~mm}^{-1}$

476

$0.31 \times 0.29 \times 0.27 \mathrm{~mm}^{3}$

1.783 to $26.425^{\circ}$.

$-10<=\mathrm{h}<=8,-12<=\mathrm{k}<=12,-15<=\mathrm{l}<=15$

13782

$3786[\mathrm{R}(\mathrm{int})=0.0308]$

$100.0 \%$

Semi-empirical from equivalents

0.432 and 0.284

Full-matrix least-squares on $\mathrm{F}^{2}$

3786 / 21 / 222

1.380

$\mathrm{R} 1=0.0350, \mathrm{wR} 2=0.0659$

$\mathrm{R} 1=0.0383, \mathrm{wR} 2=0.0666$

n/a

1.984 and -3.027 e. $\AA^{-3}$ 
Table S3. Atomic coordinates $\left(x \mathbf{1 0}^{4}\right)$ and equivalent isotropic displacement parameters $\left(\AA^{2} \times 10^{3}\right)$ for hyvl12_0m_a. U(eq) is defined as one third of the trace of the orthogonalized $U^{\mathrm{ij}}$ tensor.

\begin{tabular}{|c|c|c|c|c|}
\hline & $\mathrm{x}$ & $\mathrm{y}$ & $\mathrm{z}$ & $\mathrm{U}(\mathrm{eq})$ \\
\hline $\operatorname{Bi}(1)$ & 2066(1) & $5487(1)$ & 1371(1) & $21(1)$ \\
\hline $\mathrm{C}(1)$ & $3382(8)$ & $6758(6)$ & $521(5)$ & $23(1)$ \\
\hline$C(2)$ & $5152(8)$ & $6989(6)$ & $841(5)$ & $26(1)$ \\
\hline$C(17)$ & $-478(8)$ & $6996(6)$ & 2734(5) & $24(1)$ \\
\hline$C(16)$ & $696(7)$ & $7274(6)$ & $2172(5)$ & $24(1)$ \\
\hline $\mathrm{N}(2)$ & $9359(7)$ & $6381(6)$ & $6917(5)$ & $31(1)$ \\
\hline$C(4)$ & $5047(8)$ & $8148(6)$ & $-664(5)$ & $25(1)$ \\
\hline$C(6)$ & 2444(8) & $7239(6)$ & $-407(5)$ & $27(1)$ \\
\hline$C(12)$ & 6918(7) & $6137(6)$ & $5031(5)$ & 19(1) \\
\hline$C(5)$ & $3264(9)$ & $7942(6)$ & $-979(5)$ & $29(2)$ \\
\hline$C(18)$ & $-1325(8)$ & $8077(7)$ & $3265(5)$ & $28(1)$ \\
\hline$C(10)$ & $5570(8)$ & $4975(6)$ & $3017(5)$ & $24(1)$ \\
\hline$C(13)$ & $5642(7)$ & $7051(6)$ & $5030(5)$ & $23(1)$ \\
\hline $\mathrm{C}(9)$ & $4269(7)$ & $5869(6)$ & $3010(5)$ & $19(1)$ \\
\hline $\mathrm{C}(11)$ & $6890(7)$ & $5098(6)$ & $4018(5)$ & $22(1)$ \\
\hline $\mathrm{C}(14)$ & 4314(7) & $6917(6)$ & 4023(5) & $23(1)$ \\
\hline$C(20)$ & 183(9) & $9731(7)$ & $2681(6)$ & $37(2)$ \\
\hline$C(3)$ & 5975(8) & $7683(6)$ & $270(5)$ & $25(1)$ \\
\hline$C(22)$ & $-1790(40)$ & $10510(30)$ & $3690(20)$ & $36(3)$ \\
\hline $\mathrm{C}(21)$ & $1023(8)$ & $8648(7)$ & $2147(6)$ & $30(2)$ \\
\hline$C(7)$ & $5710(40)$ & $8730(30)$ & $-1320(20)$ & $30(3)$ \\
\hline$C(15)$ & $8284(7)$ & $6276(6)$ & $6083(5)$ & $21(1)$ \\
\hline$C(19)$ & $-999(8)$ & $9444(7)$ & $3238(6)$ & $30(2)$ \\
\hline $\mathrm{N}(3)$ & $-2459(19)$ & $11445(13)$ & $4066(12)$ & $50(3)$ \\
\hline $\mathrm{N}(1)$ & $6341(17)$ & $9108(13)$ & $-1924(10)$ & $44(3)$ \\
\hline $\mathrm{C}(8)$ & $6080(40)$ & $8900(30)$ & $-1330(30)$ & $30(3)$ \\
\hline $\mathrm{C}(23)$ & $-2000(40)$ & $10670(30)$ & $3920(20)$ & $36(3)$ \\
\hline
\end{tabular}


Table S4. Bond lengths $[\AA ̊]$ and angles $\left[^{\circ}\right]$ for hyvl12_0m_a.

\begin{tabular}{|c|c|}
\hline $\mathrm{Bi}(1)-\mathrm{C}(1)$ & $2.255(6)$ \\
\hline $\mathrm{Bi}(1)-\mathrm{C}(16)$ & $2.253(6)$ \\
\hline $\mathrm{Bi}(1)-\mathrm{C}(9)$ & $2.263(5)$ \\
\hline $\mathrm{C}(1)-\mathrm{C}(2)$ & $1.394(8)$ \\
\hline $\mathrm{C}(1)-\mathrm{C}(6)$ & $1.397(8)$ \\
\hline $\mathrm{C}(2)-\mathrm{H}(2)$ & 0.9500 \\
\hline $\mathrm{C}(2)-\mathrm{C}(3)$ & $1.381(9)$ \\
\hline $\mathrm{C}(17)-\mathrm{H}(17)$ & 0.9500 \\
\hline$C(17)-C(16)$ & $1.384(9)$ \\
\hline $\mathrm{C}(17)-\mathrm{C}(18)$ & $1.394(9)$ \\
\hline$C(16)-C(21)$ & $1.393(8)$ \\
\hline $\mathrm{N}(2)-\mathrm{C}(15)$ & $1.142(7)$ \\
\hline$C(4)-C(5)$ & $1.403(9)$ \\
\hline $\mathrm{C}(4)-\mathrm{C}(3)$ & 1.391(9) \\
\hline $\mathrm{C}(4)-\mathrm{C}(7)$ & $1.324(14)$ \\
\hline $\mathrm{C}(4)-\mathrm{C}(8)$ & $1.612(16)$ \\
\hline $\mathrm{C}(6)-\mathrm{H}(6)$ & 0.9500 \\
\hline$C(6)-C(5)$ & $1.386(9)$ \\
\hline $\mathrm{C}(12)-\mathrm{C}(13)$ & $1.389(8)$ \\
\hline $\mathrm{C}(12)-\mathrm{C}(11)$ & $1.388(8)$ \\
\hline$C(12)-C(15)$ & $1.443(7)$ \\
\hline $\mathrm{C}(5)-\mathrm{H}(5)$ & 0.9500 \\
\hline $\mathrm{C}(18)-\mathrm{H}(18)$ & 0.9500 \\
\hline $\mathrm{C}(18)-\mathrm{C}(19)$ & $1.387(9)$ \\
\hline $\mathrm{C}(10)-\mathrm{H}(10)$ & 0.9500 \\
\hline$C(10)-C(9)$ & $1.392(8)$ \\
\hline $\mathrm{C}(10)-\mathrm{C}(11)$ & $1.384(8)$ \\
\hline $\mathrm{C}(13)-\mathrm{H}(13)$ & 0.9500 \\
\hline$C(13)-C(14)$ & $1.390(8)$ \\
\hline $\mathrm{C}(9)-\mathrm{C}(14)$ & $1.390(8)$ \\
\hline $\mathrm{C}(11)-\mathrm{H}(11)$ & 0.9500 \\
\hline $\mathrm{C}(14)-\mathrm{H}(14)$ & 0.9500 \\
\hline $\mathrm{C}(20)-\mathrm{H}(20)$ & 0.9500 \\
\hline$C(20)-C(21)$ & $1.393(10)$ \\
\hline
\end{tabular}




\begin{tabular}{|c|c|}
\hline$C(20)-C(19)$ & $1.388(10)$ \\
\hline $\mathrm{C}(3)-\mathrm{H}(3)$ & 0.9500 \\
\hline$C(22)-C(19)$ & $1.321(14)$ \\
\hline $\mathrm{C}(22)-\mathrm{N}(3)$ & $1.135(11)$ \\
\hline $\mathrm{C}(21)-\mathrm{H}(21)$ & 0.9500 \\
\hline $\mathrm{C}(7)-\mathrm{N}(1)$ & $1.137(11)$ \\
\hline$C(19)-C(23)$ & $1.643(17)$ \\
\hline $\mathrm{C}(8)-\mathrm{H}(8 \mathrm{~A})$ & 0.9800 \\
\hline $\mathrm{C}(8)-\mathrm{H}(8 \mathrm{~B})$ & 0.9800 \\
\hline $\mathrm{C}(8)-\mathrm{H}(8 \mathrm{C})$ & 0.9800 \\
\hline $\mathrm{C}(23)-\mathrm{H}(23 \mathrm{~A})$ & 0.9800 \\
\hline $\mathrm{C}(23)-\mathrm{H}(23 \mathrm{~B})$ & 0.9800 \\
\hline $\mathrm{C}(23)-\mathrm{H}(23 \mathrm{C})$ & 0.9800 \\
\hline $\mathrm{C}(1)-\mathrm{Bi}(1)-\mathrm{C}(9)$ & $94.1(2)$ \\
\hline$C(16)-B i(1)-C(1)$ & $95.0(2)$ \\
\hline$C(16)-B i(1)-C(9)$ & $93.3(2)$ \\
\hline$C(2)-C(1)-B i(1)$ & $121.2(4)$ \\
\hline$C(2)-C(1)-C(6)$ & $118.2(6)$ \\
\hline$C(6)-C(1)-B i(1)$ & $120.4(4)$ \\
\hline $\mathrm{C}(1)-\mathrm{C}(2)-\mathrm{H}(2)$ & 119.2 \\
\hline $\mathrm{C}(3)-\mathrm{C}(2)-\mathrm{C}(1)$ & $121.6(6)$ \\
\hline $\mathrm{C}(3)-\mathrm{C}(2)-\mathrm{H}(2)$ & 119.2 \\
\hline $\mathrm{C}(16)-\mathrm{C}(17)-\mathrm{H}(17)$ & 119.8 \\
\hline $\mathrm{C}(16)-\mathrm{C}(17)-\mathrm{C}(18)$ & $120.4(6)$ \\
\hline $\mathrm{C}(18)-\mathrm{C}(17)-\mathrm{H}(17)$ & 119.8 \\
\hline $\mathrm{C}(17)-\mathrm{C}(16)-\mathrm{Bi}(1)$ & $118.5(4)$ \\
\hline $\mathrm{C}(17)-\mathrm{C}(16)-\mathrm{C}(21)$ & $119.2(6)$ \\
\hline $\mathrm{C}(21)-\mathrm{C}(16)-\mathrm{Bi}(1)$ & $122.3(5)$ \\
\hline$C(5)-C(4)-C(8)$ & $123.4(12)$ \\
\hline$C(3)-C(4)-C(5)$ & $118.4(6)$ \\
\hline $\mathrm{C}(3)-\mathrm{C}(4)-\mathrm{C}(8)$ & $118.1(12)$ \\
\hline$C(7)-C(4)-C(5)$ & $116.2(15)$ \\
\hline$C(7)-C(4)-C(3)$ & $125.3(15)$ \\
\hline $\mathrm{C}(1)-\mathrm{C}(6)-\mathrm{H}(6)$ & 119.8 \\
\hline$C(5)-C(6)-C(1)$ & $120.4(6)$ \\
\hline
\end{tabular}




\begin{tabular}{|c|c|}
\hline $\mathrm{C}(5)-\mathrm{C}(6)-\mathrm{H}(6)$ & 119.8 \\
\hline$C(13)-C(12)-C(15)$ & $119.9(5)$ \\
\hline $\mathrm{C}(11)-\mathrm{C}(12)-\mathrm{C}(13)$ & $120.1(5)$ \\
\hline $\mathrm{C}(11)-\mathrm{C}(12)-\mathrm{C}(15)$ & $120.0(5)$ \\
\hline $\mathrm{C}(4)-\mathrm{C}(5)-\mathrm{H}(5)$ & 119.5 \\
\hline$C(6)-C(5)-C(4)$ & $120.9(6)$ \\
\hline $\mathrm{C}(6)-\mathrm{C}(5)-\mathrm{H}(5)$ & 119.5 \\
\hline $\mathrm{C}(17)-\mathrm{C}(18)-\mathrm{H}(18)$ & 119.8 \\
\hline $\mathrm{C}(19)-\mathrm{C}(18)-\mathrm{C}(17)$ & $120.3(6)$ \\
\hline $\mathrm{C}(19)-\mathrm{C}(18)-\mathrm{H}(18)$ & 119.8 \\
\hline $\mathrm{C}(9)-\mathrm{C}(10)-\mathrm{H}(10)$ & 119.4 \\
\hline $\mathrm{C}(11)-\mathrm{C}(10)-\mathrm{H}(10)$ & 119.4 \\
\hline $\mathrm{C}(11)-\mathrm{C}(10)-\mathrm{C}(9)$ & $121.1(5)$ \\
\hline $\mathrm{C}(12)-\mathrm{C}(13)-\mathrm{H}(13)$ & 119.8 \\
\hline $\mathrm{C}(12)-\mathrm{C}(13)-\mathrm{C}(14)$ & $120.3(6)$ \\
\hline $\mathrm{C}(14)-\mathrm{C}(13)-\mathrm{H}(13)$ & 119.8 \\
\hline$C(10)-C(9)-B i(1)$ & $118.6(4)$ \\
\hline$C(14)-C(9)-B i(1)$ & $122.0(4)$ \\
\hline $\mathrm{C}(14)-\mathrm{C}(9)-\mathrm{C}(10)$ & $119.3(5)$ \\
\hline $\mathrm{C}(12)-\mathrm{C}(11)-\mathrm{H}(11)$ & 120.3 \\
\hline$C(10)-C(11)-C(12)$ & $119.3(5)$ \\
\hline $\mathrm{C}(10)-\mathrm{C}(11)-\mathrm{H}(11)$ & 120.3 \\
\hline $\mathrm{C}(13)-\mathrm{C}(14)-\mathrm{H}(14)$ & 120.1 \\
\hline$C(9)-C(14)-C(13)$ & $119.8(5)$ \\
\hline $\mathrm{C}(9)-\mathrm{C}(14)-\mathrm{H}(14)$ & 120.1 \\
\hline $\mathrm{C}(21)-\mathrm{C}(20)-\mathrm{H}(20)$ & 120.0 \\
\hline $\mathrm{C}(19)-\mathrm{C}(20)-\mathrm{H}(20)$ & 120.0 \\
\hline$C(19)-C(20)-C(21)$ & $120.0(6)$ \\
\hline$C(2)-C(3)-C(4)$ & $120.4(6)$ \\
\hline $\mathrm{C}(2)-\mathrm{C}(3)-\mathrm{H}(3)$ & 119.8 \\
\hline $\mathrm{C}(4)-\mathrm{C}(3)-\mathrm{H}(3)$ & 119.8 \\
\hline $\mathrm{N}(3)-\mathrm{C}(22)-\mathrm{C}(19)$ & $178(3)$ \\
\hline$C(16)-C(21)-C(20)$ & $120.5(7)$ \\
\hline $\mathrm{C}(16)-\mathrm{C}(21)-\mathrm{H}(21)$ & 119.7 \\
\hline $\mathrm{C}(20)-\mathrm{C}(21)-\mathrm{H}(21)$ & 119.7 \\
\hline $\mathrm{N}(1)-\mathrm{C}(7)-\mathrm{C}(4)$ & $174(3)$ \\
\hline
\end{tabular}




\begin{tabular}{ll} 
N(2)-C(15)-C(12) & $179.6(7)$ \\
$\mathrm{C}(18)-\mathrm{C}(19)-\mathrm{C}(20)$ & $119.6(6)$ \\
$\mathrm{C}(18)-\mathrm{C}(19)-\mathrm{C}(23)$ & $117.7(13)$ \\
$\mathrm{C}(20)-\mathrm{C}(19)-\mathrm{C}(23)$ & $122.7(13)$ \\
$\mathrm{C}(22)-\mathrm{C}(19)-\mathrm{C}(18)$ & $123.5(16)$ \\
$\mathrm{C}(22)-\mathrm{C}(19)-\mathrm{C}(20)$ & $116.9(16)$ \\
$\mathrm{C}(4)-\mathrm{C}(8)-\mathrm{H}(8 \mathrm{~A})$ & 109.5 \\
$\mathrm{C}(4)-\mathrm{C}(8)-\mathrm{H}(8 \mathrm{~B})$ & 109.5 \\
$\mathrm{C}(4)-\mathrm{C}(8)-\mathrm{H}(8 \mathrm{C})$ & 109.5 \\
$\mathrm{H}(8 \mathrm{~A})-\mathrm{C}(8)-\mathrm{H}(8 \mathrm{~B})$ & 109.5 \\
$\mathrm{H}(8 \mathrm{~A})-\mathrm{C}(8)-\mathrm{H}(8 \mathrm{C})$ & 109.5 \\
$\mathrm{H}(8 \mathrm{~B})-\mathrm{C}(8)-\mathrm{H}(8 \mathrm{C})$ & 109.5 \\
$\mathrm{C}(19)-\mathrm{C}(23)-\mathrm{H}(23 \mathrm{~A})$ & 109.5 \\
$\mathrm{C}(19)-\mathrm{C}(23)-\mathrm{H}(23 \mathrm{~B})$ & 109.5 \\
$\mathrm{C}(19)-\mathrm{C}(23)-\mathrm{H}(23 \mathrm{C})$ & 109.5 \\
$\mathrm{H}(23 \mathrm{~A})-\mathrm{C}(23)-\mathrm{H}(23 \mathrm{~B})$ & 109.5 \\
$\mathrm{H}(23 \mathrm{~A})-\mathrm{C}(23)-\mathrm{H}(23 \mathrm{C})$ & 109.5 \\
$\mathrm{H}(23 \mathrm{~B})-\mathrm{C}(23)-\mathrm{H}(23 \mathrm{C})$ & 109.5 \\
\hline
\end{tabular}

Symmetry transformations used to generate equivalent atoms: 
Table S5. Anisotropic displacement parameters $\left(\AA^{2} \times 10^{3}\right)$ for hyvl12_0m_a. The anisotropic displacement factor exponent takes the form: $-2 \pi^{2}\left[h^{2} a^{* 2} U^{11}+\ldots+2 h k a^{*} b^{*} U^{12}\right]$

\begin{tabular}{|c|c|c|c|c|c|c|}
\hline & $\mathrm{U}^{11}$ & $\mathrm{U}^{22}$ & $\mathrm{U}^{33}$ & $\mathrm{U}^{23}$ & $\mathrm{U}^{13}$ & $\mathrm{U}^{12}$ \\
\hline $\operatorname{Bi}(1)$ & $18(1)$ & $16(1)$ & $23(1)$ & $5(1)$ & $-5(1)$ & $-3(1)$ \\
\hline$C(1)$ & $28(3)$ & $14(3)$ & $18(3)$ & $0(2)$ & $-2(2)$ & $-1(2)$ \\
\hline$C(2)$ & $25(3)$ & $24(3)$ & $20(3)$ & $5(3)$ & $-4(3)$ & $-3(3)$ \\
\hline $\mathrm{C}(17)$ & $25(3)$ & $19(3)$ & $25(3)$ & $10(3)$ & $0(3)$ & $3(2)$ \\
\hline$C(16)$ & $16(3)$ & $21(3)$ & $26(3)$ & $6(3)$ & $-6(2)$ & $1(2)$ \\
\hline $\mathrm{N}(2)$ & $28(3)$ & $31(3)$ & $29(3)$ & $10(2)$ & $-1(2)$ & $3(2)$ \\
\hline$C(4)$ & $28(2)$ & $19(2)$ & $22(2)$ & $-4(2)$ & $9(2)$ & $1(2)$ \\
\hline$C(6)$ & $24(3)$ & $22(3)$ & $24(3)$ & $2(3)$ & $-7(3)$ & $-4(3)$ \\
\hline$C(12)$ & $13(3)$ & $22(3)$ & $22(3)$ & $10(2)$ & $1(2)$ & $0(2)$ \\
\hline$C(5)$ & $42(4)$ & $22(3)$ & $18(3)$ & $4(3)$ & $0(3)$ & $0(3)$ \\
\hline $\mathrm{C}(18)$ & $23(3)$ & $33(4)$ & $28(3)$ & $13(3)$ & $0(3)$ & $3(3)$ \\
\hline$C(10)$ & $26(3)$ & $13(3)$ & $25(3)$ & $-1(2)$ & $-1(3)$ & $1(2)$ \\
\hline$C(13)$ & $18(2)$ & $25(2)$ & $26(2)$ & $7(2)$ & $3(2)$ & $7(2)$ \\
\hline $\mathrm{C}(9)$ & $18(3)$ & $17(3)$ & $21(3)$ & $7(2)$ & $1(2)$ & $1(2)$ \\
\hline $\mathrm{C}(11)$ & $19(3)$ & $14(3)$ & $31(3)$ & $5(2)$ & $3(2)$ & $6(2)$ \\
\hline$C(14)$ & $18(2)$ & $25(2)$ & $26(2)$ & $7(2)$ & $3(2)$ & $7(2)$ \\
\hline$C(20)$ & $37(4)$ & $18(3)$ & $46(4)$ & $10(3)$ & $-8(3)$ & $0(3)$ \\
\hline$C(3)$ & $28(2)$ & $19(2)$ & $22(2)$ & $-4(2)$ & $9(2)$ & $1(2)$ \\
\hline $\mathrm{C}(22)$ & $49(7)$ & $28(5)$ & $28(8)$ & $6(5)$ & $4(5)$ & $13(3)$ \\
\hline $\mathrm{C}(21)$ & $23(3)$ & $22(3)$ & $44(4)$ & $14(3)$ & $1(3)$ & $-5(3)$ \\
\hline$C(7)$ & $38(9)$ & $24(6)$ & $23(3)$ & $-5(4)$ & $12(5)$ & $-7(6)$ \\
\hline$C(15)$ & $22(3)$ & $18(3)$ & $23(3)$ & $9(2)$ & $4(3)$ & $2(2)$ \\
\hline$C(19)$ & $29(4)$ & $24(3)$ & $31(3)$ & $9(3)$ & $-3(3)$ & $6(3)$ \\
\hline $\mathrm{N}(3)$ & $70(9)$ & $31(6)$ & $48(8)$ & $7(6)$ & $20(7)$ & $19(6)$ \\
\hline $\mathrm{N}(1)$ & $60(8)$ & $38(7)$ & $26(6)$ & $-7(5)$ & $18(5)$ & $-24(6)$ \\
\hline$C(8)$ & $38(9)$ & $24(6)$ & $23(3)$ & $-5(4)$ & $12(5)$ & $-7(6)$ \\
\hline $\mathrm{C}(23)$ & 49(7) & $28(5)$ & $28(8)$ & $6(5)$ & $4(5)$ & $13(3)$ \\
\hline
\end{tabular}


Table S6. Hydrogen coordinates $\left(x 1^{4}\right)$ and isotropic displacement parameters $\left(\AA^{2} \times 10^{3}\right)$ for hyvl12_0m_a.

\begin{tabular}{|c|c|c|c|c|}
\hline & $\mathrm{x}$ & $\mathrm{y}$ & z & $\mathrm{U}(\mathrm{eq})$ \\
\hline $\mathrm{H}(2)$ & 5808 & 6662 & 1467 & 31 \\
\hline $\mathrm{H}(17)$ & -707 & 6063 & 2758 & 29 \\
\hline $\mathrm{H}(6)$ & 1237 & 7083 & -649 & 32 \\
\hline $\mathrm{H}(5)$ & 2609 & 8289 & -1593 & 35 \\
\hline $\mathrm{H}(18)$ & -2129 & 7876 & 3648 & 34 \\
\hline $\mathrm{H}(10)$ & 5552 & 4267 & 2322 & 29 \\
\hline $\mathrm{H}(13)$ & 5678 & 7772 & 5720 & 28 \\
\hline $\mathrm{H}(11)$ & 7767 & 4478 & 4013 & 27 \\
\hline $\mathrm{H}(14)$ & 3438 & 7539 & 4027 & 28 \\
\hline $\mathrm{H}(20)$ & 419 & 10667 & 2665 & 45 \\
\hline $\mathrm{H}(3)$ & 7181 & 7843 & 517 & 29 \\
\hline $\mathrm{H}(21)$ & 1826 & 8849 & 1763 & 36 \\
\hline $\mathrm{H}(8 \mathrm{~A})$ & 7224 & 9246 & -814 & 45 \\
\hline $\mathrm{H}(8 \mathrm{~B})$ & 5476 & 9683 & -1533 & 45 \\
\hline $\mathrm{H}(8 \mathrm{C})$ & 6169 & 8215 & -2044 & 45 \\
\hline $\mathrm{H}(23 \mathrm{~A})$ & -3173 & 10637 & 3431 & 54 \\
\hline $\mathrm{H}(23 \mathrm{~B})$ & -1413 & 11594 & 4052 & 54 \\
\hline $\mathrm{H}(23 \mathrm{C})$ & -2017 & 10517 & 4667 & 54 \\
\hline
\end{tabular}




\section{References}

(1) Barton, D., Bhatnagar, N., Finet, J-P., Motherwell, W. Pentavalent Organobismuth Reagents. Part VI. Comparative Migratory Aptitudes of Aryl Groups in the Arylation of Phenols and Enols by Pentavalent Bismuth Reagents. Tetrahedron, 1986, 42(12), 3111-3122.

(2) Matano, S.; Kinoshita, M.; Suzuki, H. Synthesis and Reactions of Some Crowded Triarylbismuthines. Bull. Chem. Soc. Jpn., 1992, 65(12), 3504-3506.

(3) Deacon, G.B.; Domagala, M.; Huber, F.; Rüther, R.; Felder, P.W. Synthesis of Arylbismuth (III) Sulfonates from Triarylbismuth compounds and Arene Sulfonic Acids or Sulfur Trioxide. Inorganic Chimica Acta, 1986, 113, 43-46.

(4) Graß1, S.; Hamze, C.; Thaddäus, J. K.; Knochel, P. Copper-Catalyzed Electrophilic Thiolation of Organozinv Halides by Using $N$-Thiophthalimides Leading to Polyfunctional Thioethers. Chem. Eur. J., 2019, 25, 3752-3755.

(5) Zhu, L.; Wehmeyer, R. M.; Rieke, R. D. The Direct Formation of Functionalized Alkyl(aryl)zinc Halides by Oxidative Addition of Highly Reactive Zinc with Organic Halides and Their Reactions with Acid Chlorides, $\alpha, \beta$-Unsatured Ketones, and Allylic, Aryl, and Vinyl Halides. J. Org. Chem., 1991, 56, $1445-1453$. 